\title{
Ethnopharmacology, Phytochemistry, and Global Distribution of Mangroves-A Comprehensive Review
}

\author{
Sadeer Nabeelah Bibi ${ }^{1}$, Mahomoodally Mohamad Fawzi ${ }^{1, * \mathbb{C}}$, Zengin Gokhan ${ }^{2} \mathbb{D}$, \\ Jeewon Rajesh ${ }^{1}$, Nazurally Nadeem ${ }^{3}{ }^{D}$, Rengasamy Kannan R.R. ${ }^{4}{ }^{*}$, Albuquerque R.D.D.G. ${ }^{5}$ \\ and Shunmugiah Karutha Pandian ${ }^{4, *}$ \\ 1 Department of Health Sciences, Faculty of Science, University of Mauritius, Réduit 80835, Mauritius; \\ nabeelah.sadeer1@umail.uom.ac.mu (S.N.B.); r.jeewon@uom.ac.mu (J.R.) \\ 2 Department of Biology, Science Faculty, Selcuk University, Campus, 42250 Konya, Turkey; \\ gokhanzengin@selcuk.edu.tr \\ 3 Department of Agricultural and Food Science, Faculty of Agriculture, University of Mauritius, Réduit 80835, \\ Mauritius; n.nazurally@uom.ac.mu \\ 4 Department of Biotechnology, Science Campus, Alagappa University, Karaikudi 630 003, India \\ $5 \quad$ Universidade Federal do Rio de Janeiro (UFRJ), Rio de Janeiro, Brazil; ricardo-diego-cf@hotmail.com \\ * Correspondence: f.mahomoodally@uom.ac.mu (M.M.F.); Rengasamy@enzymeinhibitors.co.in (R.K.R.R.); \\ sk_pandian@rediffmail.com (S.K.P.)
}

Received: 30 December 2018; Accepted: 26 March 2019; Published: 18 April 2019

\begin{abstract}
Mangroves are ecologically important plants in marine habitats that occupy the coastlines of many countries. In addition to their key ecological importance, various parts of mangroves are widely used in folklore medicine and claimed to effectively manage a panoply of human pathologies. To date, no comprehensive attempt has been made to compile and critically analyze the published literature in light of its ethnopharmacological uses. This review aims to provide a comprehensive account of the morphological characteristics, ethnobotany, global distribution, taxonomy, ethnopharmacology, phytochemical profiles, and pharmacological activities of traditionally used mangroves. Out of 84 mangrove species, only 27 species were found to be traditionally used, however not all of them are pharmacologically validated. The most common pharmacological activities reported were antioxidant, antimicrobial, and antidiabetic properties. Mangroves traditionally reported against ulcers have not been extensively validated for possible pharmacological properties. Terpenoids, tannins, steroids, alkaloids, flavonoids, and saponins were the main classes of phytochemicals isolated from mangroves. Given that mangroves have huge potential for a wide array of medicinal products and drug discovery to prevent and treat many diseases, there is a dire need for careful investigations substantiated with accurate scientific and clinical evidence to ensure safety and efficient use of these plants and validate their pharmacological properties and toxicity.
\end{abstract}

Keywords: bioactive compounds; Bruguiera gymnorhiza; Rhizophora mucronata; Avicennia species; pneumatophores; traditional uses

\section{Introduction}

Medicinal plants are potential pharmacies grown in the wild and have been co-existed and co-evolved alongside human civilizations since the beginning of life on Earth. Since ancient times, human life has been revolving around plants as they were used for their curative nature to alleviate human pain and have been the focal point of many researchers since the dawn of medicine. For centuries, medicinal plants have been used as remedies for human ailments and diseases because they contain 
components of therapeutic value. With the increasing incidence and complexity of diseases threatening human health, the need for novel and effective bio-molecules is of paramount importance, which brings forward natural products/plants as the pipeline of tomorrow for drug discovery. Alarmingly, recent estimates reported that every fifth plant species found under the kingdom Plantae are threatened with extinction [1] and thus if we are not careful, they may disappear in front of our eyes due to disastrous environmental factors taking with them notable medicinal values.

Due to the long history in folklore medicine, medicinal plants have not escaped the attention of today's pharmaceutical chemists. The importance of traditional medicines has been well understood by the pharmaceutical industry since the discovery and successful development of aspirin from the symbolic Willow tree [2]. For instance, metformin, derived from Galega officinalis L., is a commonly used type 2 diabetic drug. Interestingly, a study has shown that metformin can also have potential cytotoxic effects on cancerous cells [3]. Taxol, the blockbuster anticancer drug, derived from Taxus brevifolia Nutt., showed significant effect against various types of cancers viz; ovarian, breast, lung cancer, head, and neck tumors [4]. Medicinal plants have contributed profoundly in the discovery of new compounds, and the quest is still ongoing with the aim to search for more novel biologically active metabolites from traditionally used medicinal plants.

At the time of writing, Allkin Bob from the Royal Botanic Gardens, Kew, recorded around 28, 187 plant species as medicinal plants [5]. Many of them are commonly known in the medical lore and are also extensively used in modern phytomedicine while some of them still need a thorough investigation. This review aims at elaborating and providing an overview on mangrove plants, which are traditionally known medicinal plants and have attracted much interest in the quest for novel pharmacophores.

Mangrove is a shrub or small tree that grows in coastal brackish or saline waters in muddy or rocky soils. Mangroves are halophytes, being salt tolerant, they can quickly adapt themselves in harsh coastal conditions [6]. Currently, the word 'mangrove' encompasses 84 species from 24 genera and 16 families. However, only 70 species out of the 84 are classified as true mangroves while the rest as mangrove associates [7]. Nonetheless, the difference between these two classifications is still unclear which can lead to misinterpretations. Irrespective of the classification issues, many mangrove trees are traditionally used, and several genera have attracted the attention of many scientists, particularly the genera Rhizophora, Bruguiera, and Avicennia.

Several species of mangroves have been traditionally used against a plethora of diseases. Mangroves such as Bruguiera gymnorhiza (L.) Lam, Rhizophora mucronata Lam, and Acanthus ilicifolius L. have been recognized as the three most traditionally used mangrove species. Several in vivo and in vitro studies have been conducted on many mangrove species. For instance, Avicennia germinans (L.) L. showed anti-ulcer activity, whereas B. gymnorhiza has been reported for significant antioxidant, antidiabetic, and anti-inflammatory activities. Rhizophora apiculata Blume was screened for a wide array of pharmacological activities viz; antioxidant and antimicrobial properties. R. mucronata covered a broader spectrum of biological activities, namely antidiabetic (in vivo and in vitro), antioxidant, anti-inflammatory, antimicrobial, analgesic, anti-HIV, and anticholinesterase activities. Phytochemical screenings were also conducted on various species confirming the presence of tannins, alkaloids, and steroids among others.

In terms of distribution, Indonesia is the primary source of mangroves occupying the most significant area globally [8]. These plants form a rare and unique ecosystem but are threatened since they are destroyed five times faster than tropical forests [9]. For instance, North and Central America are recognized as the most threatened mangrove regions due to coastal development, hurricanes, and aquaculture. Aquaculturing of shrimps, mud crabs, or oysters is a critical staple job for many people in Southeast Asia. However, aquaculture is recognised as a leading threat to mangroves [10]. It is considered that $90 \%$ of the mangrove forests are found in developing countries which consequently build a thin line between livelihoods and mangroves [9]. People make a living on mangroves through fishing. Achim Steiner, head of the UN environment program, mentioned that mangroves contribute to the economy for a value of $\$ 57,000$ per hectare annually [9]. 
It is increasingly acknowledged that mangrove plants are rich in natural products and new chemical compounds. Mangroves have been given a considerable extent of scientific importance worldwide as they are known for their potent activity against many diseases namely cardiovascular disease, diabetes, hypertension, and cancer. Many studies have probed into the pharmacological aspects of different mangrove species, and a wealth of literature has already emerged and published. Attempts have been made previously to validate the traditional uses of several mangrove trees using in vitro and in vivo models. Nonetheless, reports are scanty on the ethnopharmacological uses of mangroves. Thus, this review aims to provide a comprehensive insight into the morphological characteristics, ethnobotany, global distribution, taxonomy, ethnopharmacology, phytochemical profiles, and pharmacological activities of traditionally used mangroves. In addition, primary data has been analyzed to (i) compare species that were medical lore, (ii) highlight the main countries using mangroves species as a traditional remedy, (iii) compare the types of extracts, plant parts, and assays used in pharmacological validation of species, and (iv) highlight the main compounds isolated from traditionally used mangroves.

\section{Review Methodology}

Relevant literature was collected by probing scientific electronic databases namely EBSCO, Google Scholar, PubMed, and ScienceDirect and web sources such as PROSEA, PlantNET, and The Plant List. Keywords such as the different mangrove species, traditional uses, ethnobotany, ethnopharmacology, pharmacological activities, morphological characteristics, and phytochemistry were used. The manual search of ethnobotanical textbooks and related compilations were also made. Two articles in non-English languages (Persian and Thai) were also included. Information was gathered and summarized in the forms of tables wherever appropriate. For instance, Table 1 shows the local names used in countries. Table 2 distinguishes between the three dominant types of mangroves. Table 3 shows the first 20 nations with mangrove plantation. Table 4 describes the morphological characteristics of the different mangroves species as well as giving information on which family and taxonomic rank they belong to. Table 5 describes the traditional uses concerning the different parts of the mangrove plants together with information on their corresponding country of origin. Table 6 shows which mangrove species are traditionally and pharmacologically validated. Table 7 summarizes the in vivo and in vitro assays including the different types of tests done, parts of the plants used, and biological activities on both extracts and controls. Table 8 summarizes the phytochemical compounds isolated from each mangrove species.

\section{Terms, Origin, and Definition}

The term 'mangrove' is of Guarani origin, the official language of Paraguay. In the early 1610s, the word was spelled as 'mangrow' coming from Portuguese mangue or Spanish 'mangle', but later in the 1690s the term 'mangrow' turned into an English word as 'mangrove' via folk etymology. Mangroves are associated with many terms, namely mangrove forest community or mangal and mangrove ecosystem. Other terms synonymous to mangrove forest are tidal forest, coastland woodlands, mangrove swamp, tidal swamp forest, and oceanic rainforests [6,11]. For instance, the mangrove forest community or mangal is linked with microbes and fungi while animals associated with the plants form the mangrove ecosystem [12]. It is suggested that the word 'mangrove' should be referred to specific mangrove species while the word 'mangal' to the forest community instead. Table 1 represents the local names used in different countries. 
Table 1. Local names of mangroves in different countries.

\begin{tabular}{ccc}
\hline Country & Local Names & Reference \\
\cline { 1 - 2 } Netherlands & $\begin{array}{c}\text { Vloedbosschen (mangrove community), } \\
\text { mangrove (individual species) }\end{array}$ & \\
\hline United Kingdom & Mangrove & \\
\hline France & Manglier, Paletuvier & \\
\hline Germany & Mangrove & \\
\hline Madagascar & Honkalahy, Voandrano & \\
\hline Malaysia & Manggi-manggi \\
Mauritius & Manglier, Paletuvier, Mangrove \\
Comorigues & Manglar \\
\hline Spain & Mangro \\
\hline Surinam &
\end{tabular}

The discovery of mangroves happened during the time of Alexander III of Macedon commonly known as Alexander the Great from 326-324 B.C. During Alexander's Indian expedition in 325 B.C., Nearchus (admiral of Alexander the Great's army) was ordered to sail along the shores of Indus River to the Euphrates passing through the Persian Gulf. It was during the expedition that Nearchus made the first discovery of the plant 'Mangrove'. Later in 305 B.C., a Greek philosopher Theophrastus also reported and documented the existence of the mangrove vegetation in his book entitled as "Historia Plantarum" $[6,14,15]$. As a result, it is recognized that the most ancient written shreds of evidence on mangroves were documented by Nearchus and Theophrastus. Both described the plants as 'held up by their roots like a polyp', and the leaves and flowers were Rhizophora [12,14]. Consequently, in 323 B.C., the ancient Greeks became aware of three mangrove areas namely the Red Sea, the Arabian Sea, and the Persian Gulf [15].

Many tribes and indigenous people have relied heavily on mangroves as a source of raw material and medicines. For instance, the tribal group of people living in the Orinoco Delta in Venezuela known as the Warao people has been dependent on mangrove forests for approximately 7000 years. The Warao people also known as the mangrove people used the roots of these plants to build houses and boats. Thousands of years later, mangroves attracted more people across the globe and till date has maintained its valuable importance for many people and also animals [14]. Recently, a study conducted by Gardner in Madagascar showed that lemurs use mangroves as their natural habitats for sleeping and foraging [16].

For many years, mangroves have formed remarkable and highly prolific ecosystems along the coastlines of many countries around the world that are both environmentally and medicinally important [14]. Within the scope of knowledge, mangroves originate from the Indo-Malayan regions which grow most of the mangrove species around the world with Indonesia being the first country covering the most extensive mangrove area globally (Table 2) [8,17]. The propagules and seeds produced by the plants have a unique feature which helped them to float in the water. Due to this characteristic, it was easy for the mangrove species to spread by water dispersal to Central and South America through India, East Africa about 23-66 million years ago [17]. 
Table 2. Distinguishing characteristics between three dominant types of mangroves *.

\begin{tabular}{|c|c|c|c|c|}
\hline & & Red & Black & White \\
\hline \multirow{4}{*}{ Characteristics } & Leaves & $\begin{array}{l}\text { Very shiny, very pointy } \\
\text { green on both sides, green } \\
\text { on both sides }\end{array}$ & $\begin{array}{l}\text { Less shiny, pointy, grey in } \\
\text { color in bottom surface }\end{array}$ & $\begin{array}{l}\text { Shiny on both } \\
\text { sides, round }\end{array}$ \\
\hline & Roots & $\begin{array}{l}\text { Rhizophores or arc-shaped } \\
\text { prop roots, roots come out of } \\
\text { the stem and grow } \\
\text { downwards to end in the soil }\end{array}$ & $\begin{array}{l}\text { Pneumatophores or } \\
\text { pencil-like roots, roots } \\
\text { grow against gravity from } \\
\text { the soil surface }\end{array}$ & - \\
\hline & Fruits & Cigar-shaped & Teardrop-shaped & $\begin{array}{l}\text { Smallest in } \\
\text { size }\end{array}$ \\
\hline & Examples & R. mucronata, R. mangle & A. germinans, B. gymnorhiza & L.racemosa \\
\hline
\end{tabular}

* Source: Restoring Guyanas mangrove ecosystem, 2014 (http://www.mangrovesgy.org/home/index.php/2014-04-2716-39-08/types-of-mangroves).

Morphologically, a mangrove is a shrub or small tree that grows in coastal brackish or saline waters in muddy or rocky soils. Mangroves are halophytes as they are salt tolerant and are easily adapted to harsh coastal conditions due to their buttress root system or rhizophores and their aerial roots or pneumatophores [6]. Different sources defined mangroves differently. For instance, Collins dictionary defined mangrove plants as a tree growing along the coastlines or on the bank of river in tropical countries [18] while Merriam-Webster [19] defined the plant as 'any of a genus (Rhizophora, especially R. mangle of the family Rhizophoraceae) of tropical maritime trees or shrubs that send out many prop roots and form dense masses important in coastal land building and as foundations of unique ecosystems' or 'any of numerous trees (of the genera Avicennia) with growth habits like those of the true mangroves' [19]. The Cambridge dictionary defined mangroves as tropical trees growing near water developing twisted roots growing partly above the ground [20].

On the other hand, Spalding [14] defined mangroves as 'trees or large shrubs including ferns and palms growing in or adjacent to intertidal regions which can easily adapt themselves in their environment'. However, these definitions sound paradoxical since many other plants can be mistaken for mangroves. For example, Anemopsis california (lizard tail) is a herb growing in wet or shallow waters [21], Atriplex (saltbush, genus of 250-300 species) is defined as a shrub growing in salty soils, and Limonium (sea lavender, genus of 120 species) is defined as a woody shrub growing along the coasts and in salt marshes [22]. These named plants are small in size and grow in saline conditions similarly to mangroves. Accordingly, it can be pointed out that mangroves do not have an appropriate and precise definition that demarcates the plants from any other halophytes.

\section{Botanical Classification and Types of Mangroves}

All mangroves belong to the Malpighiales order consisting of 16 families, with Rhizophoraceae being the dominant family, 24 genera and 84 species in all (Figure 1). Generally, mangroves are classified as true mangroves and mangrove associates. However, the classification does not meet the consensus of all scientists and therefore remains a debatable issue. For instance, Heritiera littoralis Aiton is classified as a true mangrove by many researchers [23-26] but is recognized as a mangrove associate by Mu et al. [27]and Mukherjee et al. [28], and Tansley and Fritsch [29] mentioned that the difference between the two groups might be based on the physiological adaptation to the environment, but this hypothesis still needs to be tested.

The Rhizophoraceae family comes from the major division of Angiosperms (flowering plants) and the well-known Malpighiales order. It is estimated that there are 350, 699 flowering plants or Angiosperms with 405 families, 14,559 genera, and 951,140 species [30]. As quoted by The Plant List (2013), the Rhizophoraceae family has 18 plant genera and 142 accepted species. However, not all species from the Rhizophoraceae family are considered as true mangroves; only 24 species from the four genera Bruguiera, 
Ceriops, Kandelia, and Rhizophora are called the 'Mangrove trees' [31]. Rhizophoraceae family is known as the richest family concerning mangrove species. However, although Rhizophoraceae family encompasses many mangrove species with aerial roots, all should not be taken as the 'Mangrove family' [32].

According to Bandaranayake [33], plant species that are considered to be true mangroves could also originate from at least 17 different families. Indeed, it is reported that a total of 69 species in 27 genera belonging to 20 families are considered as true mangroves $[6,34,35]$. On the other hand, Nebula et al. [31] updated the total number of species from 69 to 84, from 27 genera to 24, and from 20 families to 16. Recently, Thatoi et al. [7] opined that among the 84 mangroves species, only 70 of them are true mangroves and the remaining 14 are mangrove associates. Therefore, it can be understood that mangrove trees do not necessarily come from the Rhizophoraceae family but can also come from other families such as Acanthaceae, Avicenniaceae, and Meliaceae, among others.

Mabberley (2008) stated that the principal genera in Rhizophoraceae are Bruguiera, Carallia, Ceriops, Crossostylis, Pellacalyx, and Rhizophora. Based on the molecular phylogenetic and floral structures analyses, it is clear that Rhizophoraceae has a sister group which is the Erythroxylaceae, from which cocaine is derived [26]. Principally, this review is based on different mangrove species of therapeutic values such as Avicennia marina (Forssk.) Vierh, Avicennia officinalis L., Avicennia ilicifolius L., B. gymnorhiza (L.) Lam, Excoecaria agallocha L., Heritiera fomes Buch.-Ham., Kandelia candel (L.) Druce, R. mucronata Lam, and Xylocarpus granatum J. Koenig among others. In Mauritius, there are only two types of mangrove species that exist, namely B. gymnorhiza and R. mucronata. Both species originate from the Rhizophoraceae family [31]. Mangroves are spread in 16 families, 24 genera, and 84 species with three main types, namely red, black, and white (Figure 1).

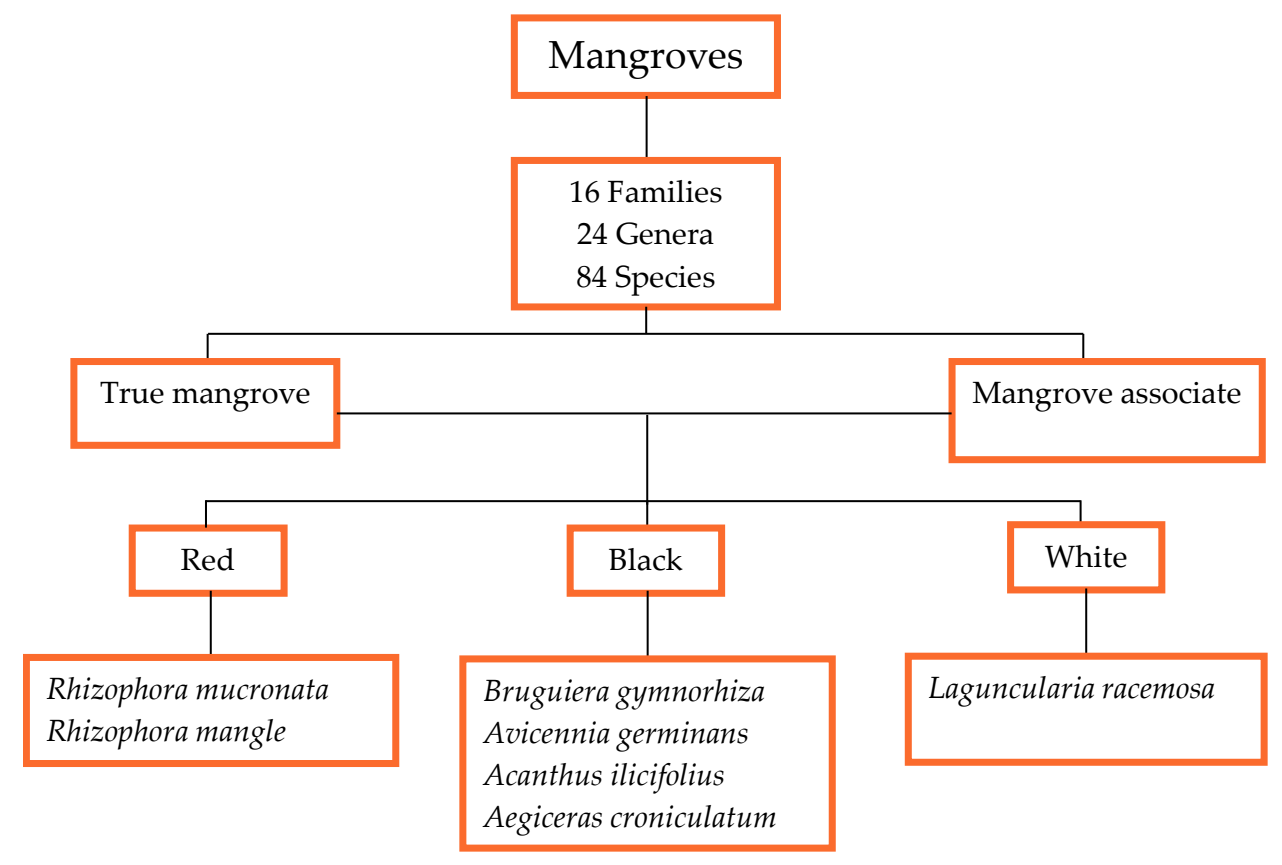

Figure 1. Classification of mangroves.

Seven types of mangrove trees exist, among which three are most dominant namely the red, black, and white mangroves. In Mauritius, two dominant types of mangrove are grown along the coastlines, namely the red (R. mucronata) and the black (B. gymnorhiza) types. Besides these two species, there is another mangrove species namely the Cassipourea gummiflua var. verticillata (N. E. Br.) J. Lewis which is scarcely cultivated in the Sir Seewoosagur Ramgoolam (SSR) Botanical Garden, Pamplemousses, Mauritius. The difference between the three most common types of mangrove (red, black, and white mangroves) is distinctive from each other based on their leaves, roots, and fruits (propagules) (http://www.mangrovesgy.org/home/index.php/2014-04-27-16-39-08/types-of-mangroves). Table 2 
describes the distinguishing characteristics between the three dominant mangrove types. However, most works of literatures have not specified or classified the mangrove species on which studies were conducted concerning their types which consequently results in only a few examples given in Table 2 and Figure 1.

Additionally, the difference is based on the tide level they survive. For instance, red mangroves grow in the low tide, black mangroves are mostly found growing in medium high tide, while white mangroves grow on a higher tide level experiencing lower tide flushing compared to the other two types (https://wetlandsandwildlife.wordpress.com/2017/03/06/featured-content-2/). Furthermore, there are other types of mangroves known as the Buttonwood, but they are not considered as true mangroves since they produce seeds instead of propagules and grow on a higher upland area compared to white mangroves (https://wetlandsandwildlife.wordpress.com/2017/03/06/featured-content-2/).

\section{Biogeographical Distribution of Mangroves}

Mangrove forests are known as the world's most productive ecosystems, and they occur mainly in the tropical or sub-tropical regions [36]. Mangroves are found in 123 countries across the globe [14]. The total area covered by mangrove trees in the world was estimated to be $137,760 \mathrm{~km}^{2}$ in 2000 [37] and currently mangroves covered about $152,000 \mathrm{~km}^{2}$ [9]. Approximately $75 \%$ of mangroves are found in 15 countries with only $6.9 \%$ of them are protected [38]. The top 20 mangrove nations in the world with Indonesia covering the largest area followed by Brazil, Malaysia, and lastly Cameroon [8] are shown in Table 3.

Table 3. Top 20 mangroves-holding nations in 2014 in $\mathrm{km}^{2}$ and percentage of global total.

\begin{tabular}{cclc}
\hline Rank & Country & $\mathbf{k m}^{\mathbf{2}}$ & $\mathbf{\%}$ Global Total \\
\hline 1 & Indonesia & 42,278 & 25.79 \\
2 & Brazil & 17,287 & 10.55 \\
3 & Malaysia & 7616 & 4.65 \\
4 & Venezuela & 7516 & 4.59 \\
5 & Nigeria & 6908 & 4.21 \\
6 & Papua New & 6236 & 3.80 \\
7 & Guinea & 6236 & 3.80 \\
8 & Colombia & 6036 & 3.68 \\
9 & Mexico & 3936 & 2.40 \\
10 & Thailand & 2.36 \\
11 & Gabon & 3864 & 2.31 \\
12 & Myanmar & 3783 & 2.02 \\
13 & Australia & 3314 & 1.63 \\
14 & Panama & 2673 & 1.62 \\
15 & Mozambique & 2658 & 1.47 \\
16 & Cuba & 2407 & 1.41 \\
17 & Bangladesh & 2314 & 1.27 \\
18 & Philippines & 2084 & 1.16 \\
19 & Ecuador & 1906 & 0.95 \\
20 & United States & 1554 & 0.81 \\
\hline
\end{tabular}

Overall, Asia consists of the largest amount of mangrove's forest (42\%) in the world followed by Africa (21\%), North/Central America (15\%), and lastly by South America (11\%).

In Mauritius, R. mucronata occupies an approximate area of $20 \mathrm{~km}^{2}$ of the coastline of Mauritius and are found mostly on the northeast, east, and southeast coastline of the island (Grand Gaube, Pointe des lascars, Poste la Fayette, Ile aux Cerfs, Trou D'eau Douce, Beau Champ, Grand Sable, Mahébourg) and is found scarcely in the south-southwest coasts (Maconde, Tamarin) [39]. There are only two predominant species of mangroves on the island namely, R. mucronata (Figure 2) and B. gymnorhiza (Figure 3) [40]. 


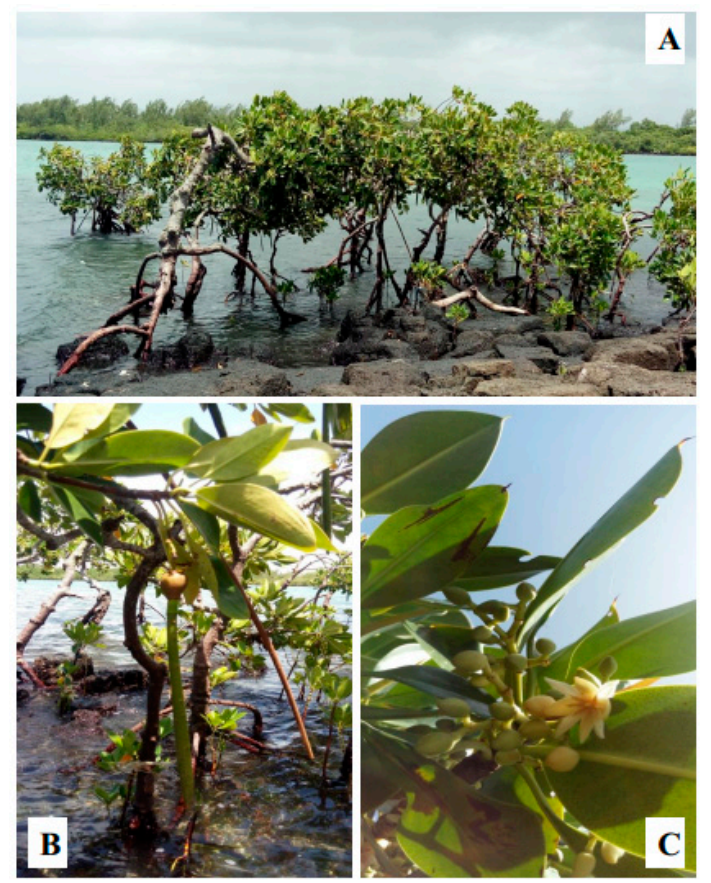

Figure 2. (A) R. mucronata growing along the coastline at Bras d'Eau public beach, Mauritius; (B) propagule; (C) flower.

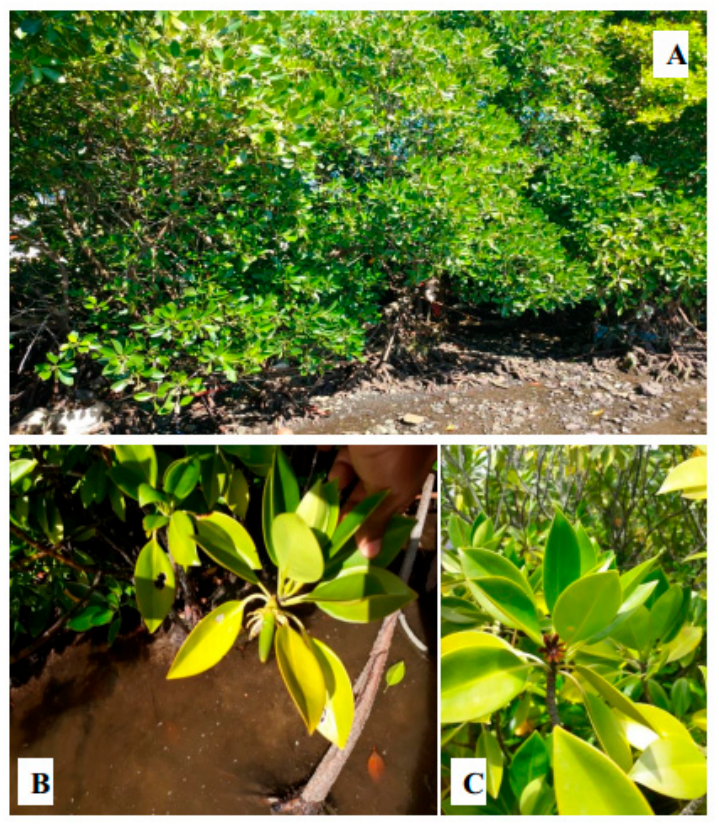

Figure 3. (A) B. gymnorhiza growing along the coastline at Bambous Virieux, Mauritius; (B) propagule; (C) flower.

Mauritius has lost 30\% of its mangrove population in seven years (1987-1994) from $20 \mathrm{~km}^{2}$ to $14 \mathrm{~km}^{2}$. Mangroves were abundantly used for firewood, construction purposes, and cut to provide a pathway for boats. In the mid-1990's, a restoration program was set up and is still active. As a result, over the past 15 years, 23 hectares of mangrove trees were restored along with approximately 230,000 seedlings [41]. 


\section{Morphological Characteristics}

The distinct morphological characteristic of mangrove plant is linked with its root systems. All mangroves have special roots known as rhizophores (buttress, stilt, or arc-shaped prop roots) or pneumatophores (pencil-like roots). These types of roots act as a respiratory system for the plants to facilitate gas exchange since mangroves grow in high saline conditions and anaerobic soils. Mangroves are called halophytes since they have good salt tolerance and filter sea water effectively for their usage. The height of the plants varies from $2 \mathrm{~m}$ to $50 \mathrm{~m}$. Their leaves are thick, elliptical in shape, and dark green in color, except for Nypa fruticans Wurmb (commonly known as Nypa palm) species which have thin long leaves resembling leaves of a palm tree. The fruits of most mangrove species have a cigar-shaped structure (long and cylindrical), green in color, and varying in length ranging from $2 \mathrm{~cm}$ to $25 \mathrm{~cm}$. Table 4 summarizes the morphological characteristics of various mangrove species. 
Table 4. Morphological characteristics of mangroves.

\begin{tabular}{|c|c|c|c|c|c|c|c|c|}
\hline Species & Family & Height & Aerial Roots & Bark & Leaves & Fruits & Flowers & Reference \\
\hline $\begin{array}{l}\text { Acanthus } \\
\text { ilicifolius L. }\end{array}$ & Acanthaceae & Up to $2 \mathrm{~m}$ & Stilt & - & Spiny edges & Kidney shaped & $\begin{array}{l}\text { Large light-violet } \\
\text { petals }\end{array}$ & [42] \\
\hline $\begin{array}{c}\text { Aegiceras } \\
\text { corniculatum (L.) } \\
\text { Blanco }\end{array}$ & Primulaceae & Up to $6 \mathrm{~m}$ & - & Smooth, greyish & $\begin{array}{l}\text { Alternate, obovate, } \\
3-10 \mathrm{~cm} \text { long, } 1.5-5 \mathrm{~cm} \\
\text { wide }\end{array}$ & $\begin{array}{l}\text { Light green to pink, } \\
\text { curved cylinder, } \\
2-7.5 \mathrm{~cm} \text { long }\end{array}$ & $\begin{array}{l}\text { Fragrant, white, } \\
\text { clusters of } 10-30\end{array}$ & [43] \\
\hline $\begin{array}{l}\text { Aegralitis } \\
\text { rotundifolia }\end{array}$ & Plumbaginaceae & $2-3 \mathrm{~m}$ & - & - & $\begin{array}{l}\text { Broad ovate, obtuse apex, } \\
5-8.8 \mathrm{~cm} \text { long, } 4.5-8.5 \mathrm{~cm} \\
\text { wide }\end{array}$ & - & - & {$[44,45]$} \\
\hline $\begin{array}{l}\text { Avicennia integra } \\
\text { N.C.Duke }\end{array}$ & Acanthaceae & $2-7 \mathrm{~m}$ & Pneumatophores & $\begin{array}{l}\text { Smooth, brown to } \\
\text { reddish }\end{array}$ & $\begin{array}{c}\text { Opposite, simple, } \\
\text { elliptical, shiny green on } \\
\text { upper surface, pale and } \\
\text { fine on bottom surface, } \\
5-14 \mathrm{~cm} \text { long }\end{array}$ & $\begin{array}{l}\text { Pale green, furry, } \\
\text { ovoid pods, } \\
21-23 \text { mm long, } \\
12-15 \mathrm{~mm} \text { wide }\end{array}$ & $\begin{array}{l}\text { Golden yellow or } \\
\text { orange, zygomorphic }\end{array}$ & [7] \\
\hline $\begin{array}{c}\text { Avicennia } \\
\text { bicolour Standl. }\end{array}$ & Acanthaceae & $8-20 \mathrm{~m}$ & - & - & - & - & $\begin{array}{l}\text { White corolla with } \\
\text { yellow throat, hairy } \\
\text { petals, zygomorphic, } \\
5-6 \mathrm{~mm} \text { in diameter }\end{array}$ & [7] \\
\hline $\begin{array}{l}\text { Avicennia marina } \\
\text { (Forssk.) Vierh. }\end{array}$ & Acanthaceae & Up to $14 \mathrm{~m}$ & Pneumatophores & $\begin{array}{l}\text { Smooth light grey } \\
\text { made up of thin, } \\
\text { stiff, brittle flakes }\end{array}$ & $\begin{array}{l}\text { Thick, bright, and glossy } \\
\text { on upper leaf, grey or } \\
\text { silvery-white on bottom } \\
\text { leaf, } 5-8 \mathrm{~cm} \text { long }\end{array}$ & $\begin{array}{l}\text { Green, oval, } \\
20-25 \mathrm{~mm} \text { in } \\
\text { diameter }\end{array}$ & $\begin{array}{l}\text { White or golden } \\
\text { yellow, clusters of 3-5 }\end{array}$ & {$[7,46]$} \\
\hline $\begin{array}{c}\text { Avicennia } \\
\text { officinalis L. }\end{array}$ & Acanthaceae & Up to $30 \mathrm{~m}$ & Pneumatophores & $\begin{array}{l}\text { Smooth, dirty green } \\
\text { to dark grey. } \\
\text { Slightly fissured } \\
\text { and does not flake }\end{array}$ & $\begin{array}{l}\text { Shiny green with round } \\
\text { apex, golden brown on } \\
\text { upper leaf, } 10 \mathrm{~cm} \text { long, } \\
5 \mathrm{~cm} \text { wide }\end{array}$ & $\begin{array}{l}\text { Green or brown, } \\
\text { heart-shaped }\end{array}$ & $\begin{array}{l}\text { Orange yellow to } \\
\text { lemon yellow, } \\
\text { 6-10 mm in diameter }\end{array}$ & [47] \\
\hline $\begin{array}{c}\text { Avicennia } \\
\text { schauerina Stapf } \\
\text { \& Leechm. ex } \\
\text { Moldenke }\end{array}$ & Acanthaceae & - & - & - & - & $\begin{array}{l}\text { Pale sap green with } \\
\text { purple tinge, flat }\end{array}$ & Slightly hairy corolla & [7] \\
\hline
\end{tabular}


Table 4. Cont

\begin{tabular}{|c|c|c|c|c|c|c|c|c|}
\hline Species & Family & Height & Aerial Roots & Bark & Leaves & Fruits & Flowers & Reference \\
\hline $\begin{array}{l}\text { Bruguiera } \\
\text { cylindrica (L.) } \\
\text { Blume }\end{array}$ & Rhizophoraceae & Up to $20 \mathrm{~m}$ & Pneumatophores & $\begin{array}{l}\text { Smooth and grey, } \\
\text { with corky raised } \\
\text { patches containing } \\
\text { lenticels (pores) }\end{array}$ & $\begin{array}{l}\text { Glossy, elliptical with } \\
\text { pointed apex }\end{array}$ & $\begin{array}{l}\text { Curved cylinder, } \\
15 \mathrm{~cm} \text { long }\end{array}$ & $\begin{array}{l}\text { Greenish white, } \\
\text { clusters of } 2-5\end{array}$ & [48] \\
\hline $\begin{array}{c}\text { Bruguiera } \\
\text { sexangula (Lour.) } \\
\text { Poir. }\end{array}$ & Rhizophoraceae & Up to $15 \mathrm{~m}$ & Pneumatophores & $\begin{array}{l}\text { Smooth, } \\
\text { grey-brown }\end{array}$ & $\begin{array}{l}\text { Smooth, glossy green with } \\
\text { pointed apex, } 9.5-20 \mathrm{~cm} \\
\text { long, } 3-7 \mathrm{~cm} \text { wide }\end{array}$ & $\begin{array}{l}\text { Green, cigar shaped, } \\
5-12 \mathrm{~cm} \text { long, } \\
1-2 \mathrm{~cm} \text { wide }\end{array}$ & $\begin{array}{l}\text { Pale yellow-green to } \\
\text { pinkish orange sepals }\end{array}$ & [43] \\
\hline $\begin{array}{c}\text { Bruguiera } \\
\text { gymnorhiza (L.) } \\
\text { Lam }\end{array}$ & Rhizophoraceae & $5-8 \mathrm{~m}$ & Pneumatophores & $\begin{array}{l}\text { Rough, } \\
\text { reddish-brown }\end{array}$ & $\begin{array}{l}\text { Large, dark green, shiny, } \\
\text { elliptical in shape with } \\
\text { reddish petiole, } \\
3-4.5 \mathrm{~cm} \text { long }\end{array}$ & $\begin{array}{l}\text { Green, cigar shaped, } \\
2 \mathrm{~cm} \text { long }\end{array}$ & $\begin{array}{c}\text { Creamy white to } \\
\text { brown }\end{array}$ & [13] \\
\hline $\begin{array}{l}\text { Ceriops tagal } \\
\text { (Perr.) C. B. } \\
\text { Robb. }\end{array}$ & Rhizophoraceae & $\mathrm{Up}$ to $25 \mathrm{~m}$ & Buttress & $\begin{array}{l}\text { Smooth, lenticels, } \\
\text { silvery-grey to } \\
\text { orangeish-brown }\end{array}$ & $\begin{array}{c}\text { Opposite in pairs, obovate, } \\
\text { yellowish-green on bottom } \\
\text { surface, } 6 \mathrm{~cm} \text { long, } \\
3 \mathrm{~cm} \text { wide }\end{array}$ & $\begin{array}{c}\text { Ovoid, } 3 \mathrm{~cm} \text { long, } \\
\text { brown }\end{array}$ & - & [49] \\
\hline $\begin{array}{l}\text { Excoecaria } \\
\text { agallocha } \mathrm{L} \text {. }\end{array}$ & Euphorbiaceae & Up to $15 \mathrm{~m}$ & $\begin{array}{c}\text { Elbow-shaped } \\
\text { pegs }\end{array}$ & - & $\begin{array}{l}\text { Alternate, elliptical, apex } \\
\text { shortly acuminate, narrow } \\
\text { base, } 3-8 \mathrm{~cm} \text { long, } \\
1.5-3 \mathrm{~cm} \text { wide }\end{array}$ & $\begin{array}{l}\text { 3-lobed, } 8 \mathrm{~mm} \text { in } \\
\text { diameter }\end{array}$ & Yellow, Unisexual & [50] \\
\hline $\begin{array}{l}\text { Heritiera fomes } \\
\text { Buch.-Ham }\end{array}$ & Sterculiaceae & $15-25 \mathrm{~m}$ & Pneumatophores & - & Elliptical & - & $\begin{array}{l}\text { Pink or orange, } \\
\text { bell-shaped, } \\
5 \mathrm{~mm} \text { across }\end{array}$ & [51] \\
\hline $\begin{array}{l}\text { Heritiera littoralis } \\
\text { Aiton }\end{array}$ & Sterculiaceae & Up to $25 \mathrm{~m}$ & Pneumatophores & - & $\begin{array}{l}\text { Dark green, short petioles } \\
\text { of } 1 \mathrm{~cm} \text {, elliptical, acute } \\
\text { apex, } 10-23 \mathrm{~cm} \text { long, } \\
4-10 \mathrm{~cm} \text { wide }\end{array}$ & $\begin{array}{l}\text { Light green to } \\
\text { brown }\end{array}$ & Unisexual & [51] \\
\hline $\begin{array}{l}\text { Kandelia candel } \\
\text { (L.) Druce }\end{array}$ & Rhizophoraceae & Up to $10 \mathrm{~m}$ & - & $\begin{array}{c}\text { Flaky, reddish } \\
\text { brown with lenticels }\end{array}$ & - & Oval, $25 \mathrm{~cm}$ long & White & [49] \\
\hline $\begin{array}{l}\text { Nypa fruticans } \\
\text { Wurmb }\end{array}$ & Arecaceae & Up to $9 \mathrm{~m}$ & - & - & Palm-like & Woody nut & $\begin{array}{c}\text { Catkin-like, red or } \\
\text { yellow }\end{array}$ & [52] \\
\hline $\begin{array}{l}\text { Pelliciera } \\
\text { rhizophorae } \\
\text { Planch. \& } \\
\text { Triana }\end{array}$ & Tetramerisataceae & Up to $20 \mathrm{~m}$ & Buttress & Brown & $\begin{array}{l}\text { Dark green, leather-like, } \\
\text { smooth on both upper and } \\
\text { bottom surface, small hairs } \\
\text { on edges, elongated, } \\
\text { pointed, } 20 \mathrm{~cm} \text { long, } \\
5 \mathrm{~cm} \text { wide }\end{array}$ & $\begin{array}{l}\text { Brown, spherical } \\
\text { with a pointed end }\end{array}$ & $\begin{array}{l}\text { 5-rayed symmetric } \\
\text { red or white petals }\end{array}$ & [53] \\
\hline
\end{tabular}


Table 4. Cont

\begin{tabular}{|c|c|c|c|c|c|c|c|c|}
\hline Species & Family & Height & Aerial Roots & Bark & Leaves & Fruits & Flowers & Reference \\
\hline $\begin{array}{c}\text { Rhizophora } \\
\text { apiculata Blume }\end{array}$ & Rhizophoraceae & Up to $30 \mathrm{~m}$ & Stilt & $\begin{array}{l}\text { Grey, almost } \\
\text { smooth, } 50 \mathrm{~cm} \\
\text { diameter }\end{array}$ & $\begin{array}{l}\text { Decussate, rosette-like at } \\
\text { end of twigs, acute apex, } \\
\text { reddish petiole, } \\
1.5-3 \mathrm{~cm} \text { long }\end{array}$ & $\begin{array}{l}\text { Brown, ovoid or } \\
\text { inversely } \\
\text { pear-shaped berry, } \\
\text { rough, } 2-3.5 \mathrm{~cm} \\
\text { long }\end{array}$ & $\begin{array}{l}\text { Yellow, bisexual, } \\
\text { 4-lobed calyx }\end{array}$ & {$[54,55]$} \\
\hline $\begin{array}{l}\text { Rhizophora } \\
\text { mangle L. }\end{array}$ & Rhizophoraceae & Up to $24 \mathrm{~m}$ & Stilt & $\begin{array}{l}\text { Grey or grey-brown, } \\
\text { smooth, thin }\end{array}$ & $\begin{array}{l}\text { Opposite, elliptical, acute } \\
\text { apex, thick, shiny green } \\
\text { on upper surface, } \\
\text { yellow-green, black dots } \\
\text { on bottom surface, } 6-12 \\
\mathrm{~cm} \text { long, } 2.5-6 \mathrm{~cm} \text { wide }\end{array}$ & - & Pale pink & {$[34,56]$} \\
\hline $\begin{array}{c}\text { Rhizophora } \\
\text { mucronata Lam. }\end{array}$ & Rhizophoraceae & $20-25 \mathrm{~m}$ & $\begin{array}{l}\text { Stilt roots } \\
\text { buttressing the } \\
\text { trunk }\end{array}$ & - & $\begin{array}{l}\text { Thick, dark green, } \\
\text { distinct mucronate tip, } \\
\text { covered with minute } \\
\text { black spots on } \\
\text { inferior surface } \\
\end{array}$ & Green, cigar-shaped & Creamy-white & [13] \\
\hline $\begin{array}{c}\text { Rhizophora } \\
\text { racemosa G. Mey }\end{array}$ & Rhizophoraceae & Up to $30 \mathrm{~m}$ & Stilt & - & $\begin{array}{l}\text { Opposite, elliptical, } \\
\text { hairless blades }\end{array}$ & - & - & $[26])$ \\
\hline $\begin{array}{l}\text { Rhizophora } \\
\text { stylosa Griff. }\end{array}$ & Rhizophoraceae & Up to $15 \mathrm{~m}$ & - & $\begin{array}{l}\text { Dark brown to } \\
\text { black }\end{array}$ & - & $\begin{array}{l}\text { Ovoid to } \\
\text { pear-shaped, } \\
4 \mathrm{~cm} \text { long }\end{array}$ & - & [57] \\
\hline $\begin{array}{l}\text { Xylocarpus } \\
\text { granatum J. } \\
\text { Koenig }\end{array}$ & Meliaceae & $3-8 \mathrm{~m}$ & Buttress long & $\begin{array}{l}\text { Light brown, } \\
\text { yellowish or } \\
\text { greenish, smooth, } \\
\text { flaky }\end{array}$ & $\begin{array}{l}\text { Bright light green to dark } \\
\text { green, round apex, } \\
\text { pinnate }\end{array}$ & - & White, $8 \mathrm{~mm}$ across & [24] \\
\hline
\end{tabular}




\section{Ethnopharmacological Uses}

Mangroves have shown potential and promising therapeutic applications to treat a variety of ailments as reported by many ethnomedicinal studies. Various parts of the plants such as the leaves, roots, barks, or stems have been used in folk medicines. They are mainly used medicinally to treat diabetes, hypertension, and gastrointestinal disorders such as constipation, diarrhea, dysentery, dyspepsia, hematuria, and stomach pain. The plants are mostly used in Asian countries, namely India (45.8\%), Bangladesh (5.1\%), Malaysia (5.1\%), China (5.1\%), Indonesia (3.4\%), Philippines (3.4\%), and other countries with $16.9 \%$. (Figure 4 ). No report is available for the traditional usage of mangroves in European countries.

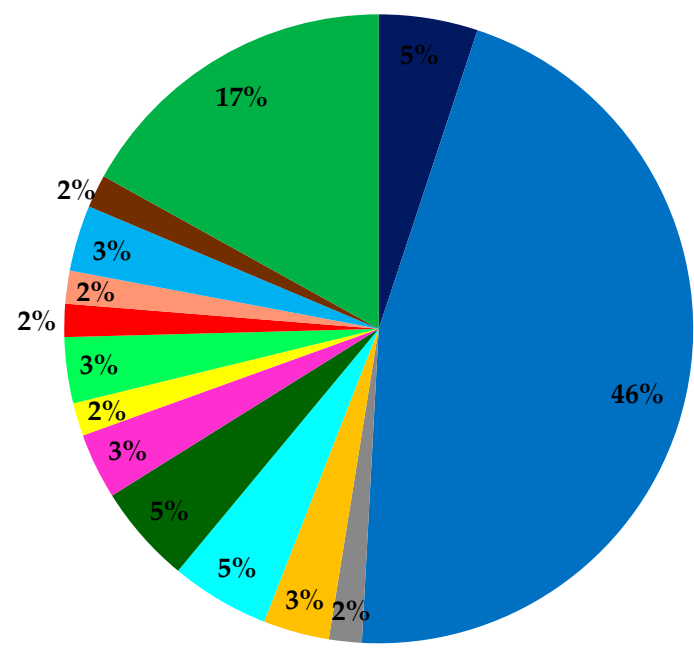

$$
\begin{aligned}
& \text { Bangladesh } \\
& \text { India } \\
& \text { South Thailand } \\
& \text { Pakistan } \\
& \text { Malaysia } \\
& \text { China } \\
& \text { Indonesia } \\
& \text { Mauritius } \\
& \text { Philippines } \\
& \text { Japan } \\
& \text { New Guinea } \\
& \square \text { Africa } \\
& \square \text { Bay of Bengal } \\
& \text { Not indicated }
\end{aligned}
$$

Figure 4. Countries using mangroves traditionally.

Species such as B. gymnorhiza (17\%), R. mucronata (14\%), A. ilicifolius (10\%), and H. fomes (9\%) are widely used traditionally and possess an array of potential medicinal values compared to the other species (Table 5 and Figure 5). For instance, A. ilicifolius is used to treat asthma, diabetes, hepatitis, leprosy, rheumatism, snake bites, among others. In India, the fruits are crushed and used as a dressing for snake bites. Additionally, the whole plant can be boiled in water, and the resulting decoction can be consumed to remove kidney stones [58]. In India, the bark decoction of X. granatum, although poorly exploited (1.85\%), is used for treating cholera and diarrhea [58].

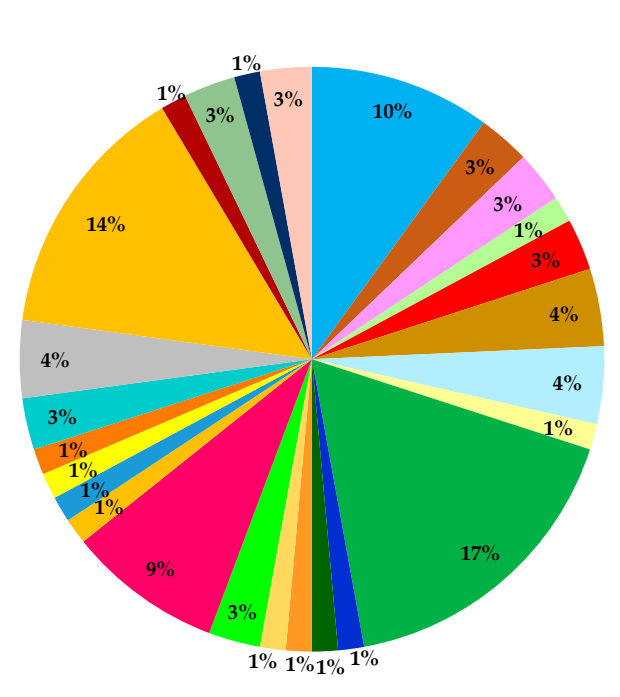

$$
\begin{aligned}
& \text { Acanthus ilicifolius } \\
& \text { Aegialitis rotundifolia } \\
& \text { Aegiceras corniculatum } \\
& \text { Acrostichum aureum } \\
& \text { Avicennia germinans } \\
& \text { Avicennia marina } \\
& \text { Avicennia officinalis } \\
& \text { Bruguiera cylindrica } \\
& \text { Bruguiera gymnorhiza } \\
& \text { Bruguiera parviflora } \\
& \text { - Ceriops decandra } \\
& \text { Ceriops roxburghiana } \\
& \text { Ceriops tagal } \\
& \text { Excoecaria agallocha } \\
& \text { - Heritiera fomes } \\
& \text { Heritiera littoralis } \\
& \text { Kandelia candel } \\
& \text { Kandelia rheedii } \\
& \text { Lumnitzera racemosa } \\
& \text { Nypa fruticans } \\
& \text { Rhizophora apiculata } \\
& \text { Rhizophora mucronata }
\end{aligned}
$$

Figure 5. Traditionally used mangrove species. 
B. gymnorhiza (Rhizophoraceae) is widely distributed in the Indian Ocean through Malaysia and Australia. The leaves and roots are mostly used in Bangladesh, China, India, and Indonesia to treat angina, diarrhea, eye disease, fever, hypertension, and intestinal worms, among others. In Comoros and Mauritius Islands, a decoction prepared from the root $(15 \mathrm{~cm}$ length) of B. gymnorhiza and five to seven leaves of Piper borbonense boiled in two cups of water, is taken twice in a day to treat haemorrhage [13]. The same decoction is also used for diabetes and hypertension.

R. mucronata (Rhizophoraceae) is commonly found in East Africa, Australia, and the Indian Ocean. R. mucronata is widely used in India. This mangrove species has tannins up to $70 \%$ of tannins which is responsible for the medicinal properties including astringent, anti-diabetic, anti-rheumatism, and hypotensive [13]. The plant is most traditionally used against diarrhea, constipation, nausea, hematuria, and diabetes. In New Guinea, it is used to cure fertility and menstruation disorders [59]. Interestingly, in Indonesia, the whole plant is used to treat elephantiasis, which is a condition caused by the enlargement of tissues due to filarial worms [60,61]. Both Bruguiera and Rhizophora genera are known to be useful for treating a wide array of diseases such as angina, haemorrhage, hematuria, and interestingly, mature leaves and roots can be used for childbirth [62], ulcers [63], diarrhea, fever, burns [64], and stings of poisonous fish [13]. Table 5 summarizes and gives a greater insight into the traditional uses of different mangrove species in different countries. 
Table 5. Traditional uses of mangrove species.

\begin{tabular}{|c|c|c|c|c|}
\hline Species & Region/Country & Plant Part(s) & Use(s) in Traditional Medicine & References \\
\hline \multirow{9}{*}{ Acanthus ilicifolius L. } & Bangladesh & WP & $\begin{array}{l}\text { Aphrodisiac, rheumatism, relief for asthma, diabetes, diuretic, dyspepsia, } \\
\text { leprosy, hepatitis, blood purifier, cure for cold, gangrenous wounds, skin } \\
\text { allergies, snake bites }\end{array}$ & {$[33,65]$} \\
\hline & West Bengal & NI & Analgesic, wound healing effect & {$[66]$} \\
\hline & NI & $\mathrm{L}$ & Pain reliever & [67] \\
\hline & Sundarbans, India & $\mathrm{L}$ & Rheumatism, neuralgia, snake bite, paralysis, asthma & [68] \\
\hline & NI & WP & Aphrodisiac, astringent, rheumatic pain, leucorrhea & {$[69]$} \\
\hline & \multirow{2}{*}{ Pichavaram, India } & $\mathrm{F}$ & Snake bites & [58] \\
\hline & & WP & Detoxification, kidney stone, small pox, skin diseases, ulcer & [70] \\
\hline & South Thailand & NI & Rheumatism, asthma, paralysis, psoriasis, leucorrhea & [71] \\
\hline & Thailand & $\mathrm{L}$ & Blood purifier, dressing against snake bites, rheumatism & \\
\hline \multirow{2}{*}{ Aegialitis rotundifolia Roxb. } & NI & $\mathrm{L}$ & Pain reliever, inflammation treatment, anti-ache agent & [72] \\
\hline & Bangladesh & $\mathrm{L}$ & Antidote for insect bites, pyrexia & [73] \\
\hline \multirow{2}{*}{$\begin{array}{c}\text { Aegiceras corniculatum (L.) } \\
\text { Blanco }\end{array}$} & Sindh, Pakistan & St & Rheumatism, painful arthritis, inflammation & [74] \\
\hline & Sindh, Pakistan & NI & Inflammatory diseases & [75] \\
\hline Acrostichum aureum $\mathrm{L}$. & Kerala, India & WP & Astringent in hemorrhage, worm remedy & [76] \\
\hline \multirow[t]{2}{*}{ Avicennia germinans (L.) L } & NI & $\mathrm{B}, \mathrm{L}, \mathrm{F}$ & $\begin{array}{c}\text { Astringent, malaria, hemorrhoids, treatment for hemorrhage, rheumatism, } \\
\text { swellings, throat ailments }\end{array}$ & [7] \\
\hline & NI & NI & Diarrhea, hemorrhage, rheumatism, hemorrhoids, tumors, swellings & [77] \\
\hline \multirow{2}{*}{ Avicennia marina (Forssk.) Vierh. } & $\mathrm{NI}$ & $\mathrm{B}, \mathrm{L}$ & Small pox, skin diseases, treatment for ulcers, throat pains & [65] \\
\hline & Iran & $\mathrm{L}$ & Ulcers, rheumatism, burns & [78] \\
\hline
\end{tabular}


Table 5. Cont.

\begin{tabular}{|c|c|c|c|c|}
\hline Species & Region/Country & Plant Part(s) & Use(s) in Traditional Medicine & References \\
\hline \multirow{7}{*}{ Avicennia officinalis L. } & \multirow{5}{*}{ Tamil Nadu, India } & $\mathrm{F}$ & Tumor, boil & \multirow{5}{*}{ [79] } \\
\hline & & $\mathrm{S}$ & Inflammation, ulcer & \\
\hline & & $\mathrm{R}$ & Aphrodisiac & \\
\hline & & $\mathrm{B}$ & Skin disease (scabies), contraceptive, astringent, hepatitis & \\
\hline & & $\operatorname{Re}$ & Snake bite, wound healing, contraceptive & \\
\hline & Tamil Nadu, India & $\mathrm{L}$ & $\begin{array}{l}\text { Asthma, paralysis, dyspepsia, rheumatism, ulcer, snake bite, skin disease, } \\
\text { small pox sores, tumor }\end{array}$ & {$[80]$} \\
\hline & Pichavaram, India & $\mathrm{L}$ & $\begin{array}{c}\text { Asthma, bronchial, detoxification, joints pain, stomach disorders, urinary } \\
\text { disorders }\end{array}$ & [58] \\
\hline Bruguiera cylindrica (L.) Blume & NI & $\mathrm{B}$ & Hemorrhage, ulcers & [63] \\
\hline \multirow{13}{*}{ Bruguiera gymnorhiza (L.) Lam } & Sundarbans, India & $\mathrm{B}, \mathrm{L}$ & Diarrhea, fever & [81] \\
\hline & India & $\mathrm{B}, \mathrm{R}$ & Diabetes, viral fever & [82] \\
\hline & Selangor, Malaysia & St & Burns, intestinal worms, liver disorders & [83] \\
\hline & $\begin{array}{l}\text { Guangxi Province, } \\
\text { China }\end{array}$ & $\mathrm{L}$ & Diarrhea & {$[54,65]$} \\
\hline & China & $\mathrm{F}$ & Shingles, eye disease, malaria & {$[84]$} \\
\hline & Indonesia & $\mathrm{F}$ & Angina, hemorrhage, hematuria & [85] \\
\hline & \multirow{3}{*}{$\begin{array}{l}\text { South Andaman } \\
\text { Island }\end{array}$} & $\mathrm{L}, \mathrm{R}$ & Eye diseases, shingles & \multirow{2}{*}{ [62] } \\
\hline & & $\mathrm{F}$ & Diarrhea, malaria, burns & \\
\hline & & $\mathrm{B}, \mathrm{R}, \mathrm{L}$ & Diabetes, hemorrhage, hypertension, stings of toxic lagoon fish & [13] \\
\hline & Comoros, Mauritius & $\mathrm{R}$ & Eye disease & [86] \\
\hline & $\begin{array}{l}\text { Pichavaram forest, } \\
\text { India }\end{array}$ & $\mathrm{L}$ & Constipation & [58] \\
\hline & Pichavaram, India & WP & \multirow{2}{*}{ Diarrhea, fever, burns, intestinal worms } & \multirow{2}{*}{ [64] } \\
\hline & NI & $\mathrm{B}, \mathrm{L}, \mathrm{F}$ & & \\
\hline
\end{tabular}


Table 5. Cont.

\begin{tabular}{|c|c|c|c|c|}
\hline Species & Region/Country & Plant Part(s) & Use(s) in Traditional Medicine & References \\
\hline $\begin{array}{l}\text { Bruguiera parviflora (Roxb.) } \\
\text { Wight \& Arn. ex Griff. }\end{array}$ & NI & B & Diabetes & [61] \\
\hline $\begin{array}{l}\text { Ceriops decandra (Griff.) W. } \\
\text { Theob. }\end{array}$ & Tamil Nadu, India & $\mathrm{B}, \mathrm{F}, \mathrm{L}$ & Hepatitis, ulcers & [87] \\
\hline Ceriops roxburghiana Arn. & NI & WP & Diabetes, ulcers & [88] \\
\hline Ceriops tagal (Perr.) C. B. Rob. & NI & B & Hemorrhage & [61] \\
\hline \multirow{2}{*}{ Excoecaria agallocha $\mathrm{L}$. } & $\mathrm{NI}$ & $\mathrm{NI}$ & Epilepsy, ulcers, leprosy, rheumatism, paralysis & [50] \\
\hline & Pichavaram, India & $\mathrm{La}$ & Toothache & [58] \\
\hline \multirow{4}{*}{ Heritiera fomes Buch.-Ham. } & Bhitarkanika, India & $\mathrm{L}, \mathrm{R}, \mathrm{S}$ & \multirow{2}{*}{$\begin{array}{c}\text { Cardiovascular diseases, gastrointestinal disorders, skin diseases, hepatic } \\
\text { disorders, gastrointestinal disorders (diarrhea, dysentery, constipation, } \\
\text { stomach ache, dyspepsia), skin diseases (rash, eczema, boils, itch, sores, } \\
\text { scabies), infections, jaundice, hepatitis, wound healing, diabetes, goiter } \\
\text { (hyperthyroidism) }\end{array}$} & {$[7,89,90]$} \\
\hline & Sundarbans, India & WP & & [51] \\
\hline & Sundarbans, India & WP & $\begin{array}{c}\text { Heart disease, bloating, stomach ache, diabetes, pain, diarrhea, skin } \\
\text { disease, hepatic disorders, goiter }\end{array}$ & [91] \\
\hline & NI & $\mathrm{T}$ & Toothache, oral infection & [69] \\
\hline \multirow{2}{*}{ Heritiera littoralis Aiton } & \multirow{2}{*}{ Philippines } & Sa & Fish, arrowhead, and spearhead poisoning & \multirow{2}{*}{ [92] } \\
\hline & & $\mathrm{S}$ & Diarrhea, dysentery, hematuria & \\
\hline Kandelia candel (L.) Druce. & NI & NI & Cardiovascular disease, cancer, neurodegenerative disorders & [93] \\
\hline Kandelia rheedii Wight \& Arn. & India & NI & Tuberculosis & [94] \\
\hline Lumnitzera racemosa Willd. & Orissa, India & $\mathrm{NI}$ & $\begin{array}{c}\text { Snake bites, rheumatism, skin allergies, blood purifier, asthma, diabetes, } \\
\text { anti-fertility }\end{array}$ & [95] \\
\hline \multirow{2}{*}{ Nypa fruticans Wurmb } & Malaysia & $\mathrm{NI}$ & Diabetes & [96] \\
\hline & Philippines & F, L & Diabetes, snake bite & {$[61]$} \\
\hline \multirow{2}{*}{ Rhizophora apiculata Blume } & Tamil Nadu, India & WP & Prevent colitis, inflammatory bowel disease (IBD) & {$[97,98]$} \\
\hline & Pichavaram, India & B & Amoebiasis, diarrhea, nausea, vomiting & {$[58]$} \\
\hline
\end{tabular}


Table 5. Cont.

\begin{tabular}{|c|c|c|c|c|}
\hline Species & Region/Country & Plant Part(s) & Use(s) in Traditional Medicine & References \\
\hline \multirow{13}{*}{ Rhizophora mucronata Lam. } & India & WP & Angina, dysentery, hematuria, hepatitis, ulcers, diabetes, hemorrhage & [98] \\
\hline & Tamil Nadu, India & B & Diarrhea, nausea, vomiting, amoebiasis, antiseptic, stop bleeding & [58] \\
\hline & Mauritius & $\mathrm{L}, \mathrm{R}$ & Astringent, antidote against toxic fish stings, diabetes, fever, hypertension & [13] \\
\hline & Porong, Indonesia & WP & Elephantiasis, hematoma, hepatitis, ulcer, febrifuge & {$[60,61]$} \\
\hline & India & $\mathrm{L}, \mathrm{R}$ & Angina, blood in urine, diabetes, diarrhea, dysentery, fever & [99] \\
\hline & Malaysia & $\mathrm{L}, \mathrm{R}$ & Childbirth, hemorrhage & \multirow{4}{*}{ [100] } \\
\hline & China & $\mathrm{B}$ & Diarrhea & \\
\hline & Japan & B & Diarrhea & \\
\hline & $\mathrm{NI}$ & $\mathrm{L}$ & Astringent, antiseptic & \\
\hline & NI & WP & Diarrhea, elephantiasis, hematuria & [33] \\
\hline & New Guinea & St & Constipation, cure fertility, menstruation disorders & [59] \\
\hline & Pichavaram, India & B & Diarrhea, nausea, vomiting & [58] \\
\hline & Thailand & B & Diarrhea, dysentery, leprosy & [71] \\
\hline Rhizophora conjugata $\mathrm{L}$. & India & $\mathrm{B}$ & Diabetes & [101] \\
\hline Rhizophora mangle L. & India & $\mathrm{B}, \mathrm{L}$ & Diabetes & {$[88,101]$} \\
\hline \multirow{2}{*}{ Rhizophora racemosa G. Mey. } & Nigeria & $\mathrm{L}$ & Toothache, dysmenorrhea & [102] \\
\hline & NI & NI & Malaria & [103] \\
\hline \multirow{6}{*}{ Xylocarpus granatum J.Koenig } & NI & NI & Cholera, diarrhea, elephantiasis, inflammation, pain, swelling of breasts & \multirow{4}{*}{ [104] } \\
\hline & East Africa & $\mathrm{B}$ & Cholera, diarrhea, fever, malaria & \\
\hline & South East Asia & $\mathrm{L}$ & Diarrhea & \\
\hline & Indian coastal region & F & Diarrhea, dyslipidemia, hyperglycemia & \\
\hline & Pichavaram, India & $\mathrm{B}$ & Cholera, diarrhea, dysentery & [58] \\
\hline & Thailand & B & Cholera & [71] \\
\hline
\end{tabular}

B = Bark, F = Fruit, La = Latex, L = Leaves, Re = Resin, R = Root, Sa = Sap, S = Seed, St = Stem, T = Twig, WP = Whole plant, NI = Not indicated. 


\section{Pharmacological Activities}

The importance of mangroves in the medical field for curing diseases cannot be undermined as the plants have much therapeutic potential. Mangroves were used in folklore medicines a long time ago, and different extracts from various parts of the plants (roots, leaves, fruits, bark, and resin) have shown exciting and significant inhibitory activities in many assays namely antidiabetic, anti-inflammatory, anti-cancer, anti-ulcer, anti-tumor, anti-viral, antioxidant, and antimicrobial among others. Since various parts of the plants were used for inhibitory assays and considering the fact that mangrove ecosystems are known to be threatened, it can be said that plant samples were being used sustainably. Although many mangrove species have been used traditionally by local inhabitants for an extended period following folk traditions in various countries as ailments, many among them have not been studied extensively yet, and thus their medicinal properties have not been reported. For example, in Mauritius, local people use the root decoction of $R$. mucronata against diabetes, but the plant has not been locally validated by researchers to confirm its pharmacological properties. Similarly, no scientific research has been carried out so far on Ceriops tagal and Kandelia rheedii to prove their efficacy against diseases that can be cured by folk medicine. Interestingly, although few studies have been conducted on the species Bruguiera sexangula, Rhizophora stylosa, and Pelliciera rhizophorae, these species are yet to be used in folk medicine (Table 6). Therefore, mangrove species require more attention from researchers to shed more light into the traditional and pharmacological uses of these unique plants as there is a dearth of knowledge on this particular area. Table 6 shows the number of species used in folklore medicines and those that are pharmacologically tested.

Table 6. Traditionally used and pharmacologically validated species of mangroves.

\begin{tabular}{|c|c|c|}
\hline Species & Traditionally Used & Pharmacologically Validated \\
\hline Acanthus ilicifolius & $\checkmark$ & $\checkmark$ \\
\hline Aegiceras rotundifolia & $\sqrt{ }$ & $\checkmark$ \\
\hline Aegiceras corniculatum & $\sqrt{ }$ & $\checkmark$ \\
\hline Acrostichum aureum & $\sqrt{ }$ & $\sqrt{ }$ \\
\hline Avicennia germinans & $\checkmark$ & $\checkmark$ \\
\hline Avicennia marina & $\checkmark$ & $\checkmark$ \\
\hline Avicennia officinalis & $\sqrt{ }$ & $\checkmark$ \\
\hline Bruguiera cylindrica & $\checkmark$ & $\sqrt{ }$ \\
\hline Bruguiera gymnorhiza & $\checkmark$ & $\checkmark$ \\
\hline Bruguiera parviflora & $\checkmark$ & $\checkmark$ \\
\hline Bruguiera sexangula & $\sqrt{ }$ & $\sqrt{ }$ \\
\hline Ceriops decandra & $\sqrt{ }$ & $\checkmark$ \\
\hline Ceriops roxburghiana & $\sqrt{ }$ & $\sqrt{ }$ \\
\hline Ceriops tagal & $\checkmark$ & $x$ \\
\hline Excoecaria agallocha & $\checkmark$ & $\checkmark$ \\
\hline Heritiera fomes & $\checkmark$ & $\checkmark$ \\
\hline Heritiera littoralis & $\sqrt{ }$ & $\sqrt{ }$ \\
\hline Kandelia candel & $\checkmark$ & $\checkmark$ \\
\hline Kandelia rheedii & $\sqrt{ }$ & $x$ \\
\hline Lumnitzera racemosa & $\checkmark$ & $\checkmark$ \\
\hline Nypa fruticans & $\sqrt{ }$ & $\sqrt{ }$ \\
\hline Pelliciera rhizophorae & $x$ & $\sqrt{ }$ \\
\hline Rhizophora apiculata & $\checkmark$ & $\sqrt{ }$ \\
\hline Rhizophora mucronata & $\checkmark$ & $\checkmark$ \\
\hline Rhizophora stylosa & $x$ & $\sqrt{ }$ \\
\hline Rhizophora conjugata & $\sqrt{ }$ & $\sqrt{ }$ \\
\hline Rhizophora mangle & $\sqrt{ }$ & $\checkmark$ \\
\hline Rhizophora racemosa & $\checkmark$ & $\checkmark$ \\
\hline Xylocarpus granatum & $\checkmark$ & $\checkmark$ \\
\hline Total number of species & 27 & 27 \\
\hline
\end{tabular}


It has been acknowledged that out of the 84 mangrove species that exist, only 26 species were mentioned in literature to possess folklore medicinal importance. However, it could be possible that the remaining 58 species have an equally influential role in the management of diseases, but due to a lack of interest, there is a dearth of knowledge of all the mangrove species. Table 7 represents the pharmacological activities of various mangrove species studied and gives a broader knowledge on the pharmacological importance on mangroves and on the different types of assays conducted. Figure 6 illustrates the types of extracts commonly used for these assays.

Methanolic extracts $(32.46 \%)$ were the most preferred extracts used in most studies followed by ethanolic $(12.28 \%)$, ethyl acetate $(10.53 \%)$, aqueous $(7.89 \%)$, and chloroform $(6.14 \%)$ (Figure 6$)$. The percentage was calculated as per report per species mentioned in Table 7.

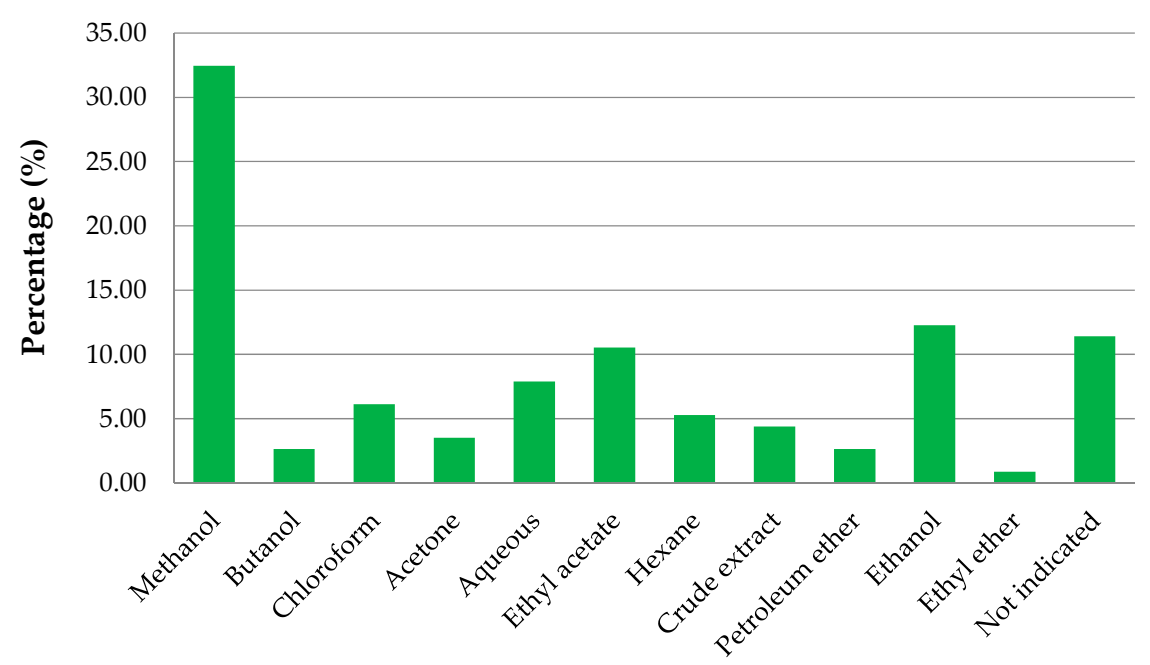

Figure 6. Types of mangrove extracts used in inhibition assays.

On the other hand, Figure 7 illustrates the types of plant parts most commonly used in the studies mentioned in Table 6. From the data shown, it can be suggested that the plant parts mostly studied are leaves $(64 \%)$, roots $(10 \%)$, stem bark (5\%), and stem $(5 \%)$. Only one work, published by Mondal et al. (2016), used latex and seed as plant samples to carry out anti-inflammatory, anticancer, analgesic, and anti-filarial activities and Wei et al. [93] conducted a test on the hypocotyl part to determine antioxidant property.

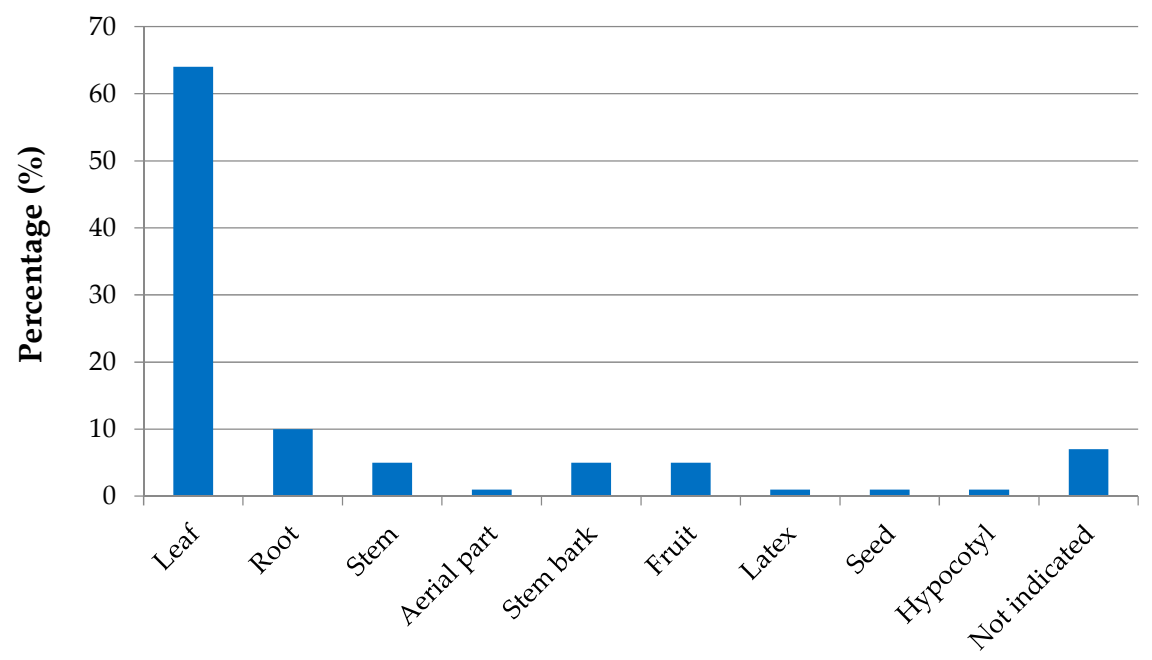

Figure 7. Types of plant parts of mangroves used in inhibition assays. 
Table 7. Pharmacological activities of different mangrove species.

\begin{tabular}{|c|c|c|c|c|c|}
\hline Species & Plant Part(s) & Extract & Study/Assays & Activity & Reference \\
\hline \multirow{14}{*}{ Acanthus ilicifolius L. } & $\mathrm{L}, \mathrm{R}$ & $\mathrm{Me}$ & $\begin{array}{l}\text { Antioxidant-DPPH } \\
\text { (In vitro) }\end{array}$ & $\mathrm{IC}_{50}(\mathrm{mg} / \mathrm{mL}): \mathrm{L}=2501.53 \pm 182.62, \mathrm{R}=1319.66 \pm 150.76$ & \multirow{2}{*}{ [105] } \\
\hline & $\mathrm{L}, \mathrm{R}$ & $\mathrm{Me}$ & $\begin{array}{l}\text { Antioxidant-FRAP } \\
\text { (In vitro) }\end{array}$ & $\operatorname{AAE}(\mathrm{mg} / \mathrm{g}): \mathrm{L}=1.10 \pm 0.03, \mathrm{R}=1.62 \pm 0.03$ & \\
\hline & $\mathrm{L}$ & $\mathrm{Me}$ & $\begin{array}{l}\text { Antinociceptive- Acetic } \\
\text { acid-induced writhing test } \\
\text { (In vivo) }\end{array}$ & $\begin{array}{l}\text { Control }(10 \mathrm{~mL} / \mathrm{kg}) \text { number of writhings }=51.5 \pm 4.1 \text {, at } 250 \text { and } \\
500 \mathrm{mg} / \mathrm{kg} \text { (extract), \%inhibition }=33.0 \% \text { and } 51.1 \% \text { respectively }\end{array}$ & \multirow[t]{2}{*}{ [68] } \\
\hline & $\mathrm{L}$ & $\mathrm{Me}$ & $\begin{array}{l}\text { Antinociceptive-Formalin test } \\
\text { (In vivo) }\end{array}$ & $\begin{array}{c}\text { At } 250 \text { and } 500 \mathrm{mg} / \mathrm{kg} \text {, \%inhibition }=37.54 \text { and } 50.18 \text { respectively for } \\
5 \mathrm{~min} \text { and } 45.5 \% \text { and } 67.24 \% \text { respectively for } 30 \mathrm{~min}\end{array}$ & \\
\hline & $\mathrm{L}$ & $\mathrm{Me}$ & $\begin{array}{l}\text { Anti-inflammatory- } \\
\text { Carrageenan-induced paw } \\
\text { edema (In vivo) }\end{array}$ & $\begin{array}{l}\mathrm{ED}_{50}(\mathrm{mg} / \mathrm{kg})=146.2,95 \% \mathrm{Cl}=69.38-286.2 \text { both at early and late } \\
\text { phases. After } 2 \mathrm{~h}, \mathrm{ED}_{50}(\mathrm{mg} / \mathrm{kg})=194,95 \% \mathrm{Cl}=135.8-301.4 \text {. With } \\
\text { BW755C (COX-LOX inhibitor) the paw edema decreased significantly. } \\
\text { No significant inhibitory activity was shown with indomethacin }\end{array}$ & \multirow{6}{*}{ [66] } \\
\hline & $\mathrm{L}$ & $\mathrm{Me}$ & $\begin{array}{l}\text { Anti-inflammatory- Acetic } \\
\text { acid-induced peritoneal } \\
\text { inflammation (In vivo) }\end{array}$ & At 200 and $400 \mathrm{mg} / \mathrm{kg}, \%$ inhibition $=48$ and 77 , respectively & \\
\hline & $\mathrm{L}$ & $\mathrm{Me}$ & Antioxidant-DPPH (In vitro) & $\begin{array}{c}\mathrm{IC}_{50}(\mathrm{~g} / \mathrm{mL}): \text { extract }=8.40 \pm 0.06, \text { Quercetin }=5.28 \pm 0.08 \\
\text { Vitamin } \mathrm{C}=6.62 \pm 0.05\end{array}$ & \\
\hline & $\mathrm{L}$ & $\mathrm{Me}$ & Antioxidant- ABTS (In vitro) & $\begin{array}{c}\mathrm{IC}_{50}(\mathrm{~g} / \mathrm{mL}): \text { extract }=10.34 \pm 0.02, \text { Quercetin }=3.60 \pm 0.03 \\
\text { Vitamin } \mathrm{C}=4.86 \pm 0.03\end{array}$ & \\
\hline & $\mathrm{L}$ & $\mathrm{Me}$ & Antioxidant- SO (In vitro) & $\begin{array}{c}\mathrm{IC}_{50}(\mathrm{~g} / \mathrm{mL}): \text { extract }=78.12 \pm 2.51, \text { Quercetin }=30.19 \pm 1.32 \\
\text { Vitamin } C=52.18 \pm 3.14\end{array}$ & \\
\hline & $\mathrm{L}$ & $\mathrm{Me}$ & Antioxidant- HO (In vitro) & $\begin{array}{c}\mathrm{IC}_{50}(\mathrm{~g} / \mathrm{mL}): \text { extract }=24.60 \pm 1.10, \text { Quercetin }=14.32 \pm 0.52 \\
\text { Vitamin } C=21.08 \pm 0.34\end{array}$ & \\
\hline & $\mathrm{L}$ & A & \multirow{3}{*}{ Antimicrobial (In vitro) } & Zone of inhibition $(\mathrm{mm})$ against $\mathrm{BS}=20, \mathrm{SA}=18, \mathrm{PA}=18, \mathrm{CA}=22$ & \multirow{3}{*}{ [67] } \\
\hline & $\mathrm{L}$ & $\mathrm{Bu}$ & & Zone of inhibition $(\mathrm{mm})$ against $\mathrm{BS}=16, \mathrm{SA}=8, \mathrm{PA}=10, \mathrm{CA}=15$ & \\
\hline & $\mathrm{L}$ & $\mathrm{C}$ & & Zone of inhibition $(\mathrm{mm})$ against $\mathrm{BS}=22, \mathrm{SA}=21, \mathrm{PA}=20, \mathrm{CA}=26$ & \\
\hline & $\mathrm{L}$ & A & $\begin{array}{l}\text { Antimicrobial-Disc diffusion } \\
\text { assay (In vitro) }\end{array}$ & $\begin{array}{c}\text { Active against EC, AGT, STM, SA, AF, and TR. Zone of inhibition } \\
(\mathrm{mm})=7.5 \pm 0.4,8 \pm 0.5,7 \pm 0.1,8.2 \pm 0.3,8.0 \pm 0.7 \text { and } 7.9 \pm 0.3 \\
\text { respectively. Me and EA extracts are inactive against TR }\end{array}$ & [106] \\
\hline
\end{tabular}


Table 7. Cont.

\begin{tabular}{|c|c|c|c|c|c|}
\hline Species & Plant Part(s) & Extract & Study/Assays & Activity & Reference \\
\hline \multirow{8}{*}{$\begin{array}{l}\text { Aegialitis rotundifolia } \\
\text { Roxb. }\end{array}$} & $\mathrm{L}$ & $\mathrm{Aq}$ & $\begin{array}{l}\text { Anti-inflammatory- Cotton } \\
\text { pellet-induced granuloma } \\
\text { (In vitro) }\end{array}$ & $\begin{array}{c}\text { At } 400 \mathrm{mg} / \mathrm{kg}, \% \text { inhibition }= \\
=63.22 \%\end{array}$ & \multirow{4}{*}{ [73] } \\
\hline & $\mathrm{L}$ & $\mathrm{Aq}$ & $\begin{array}{l}\text { Anti-inflammatory- } \\
\text { Carrageenan induced hind } \\
\text { paw edema (In vitro) }\end{array}$ & $\begin{array}{c}\text { At } 400 \mathrm{mg} / \mathrm{kg}, \% \text { inhibition }=26.75 \% \text {, while } \% \text { inhibition of } \\
\text { indomethacin }=40.13 \%\end{array}$ & \\
\hline & $\mathrm{L}$ & $\mathrm{Aq}$ & $\begin{array}{l}\text { Analgesic- Acetic acid } \\
\text { induced writhing test } \\
\text { (In vitro) }\end{array}$ & At 200 and $400 \mathrm{mg} / \mathrm{kg}$, \%inhibition $=47.86 \%$ and $57.1 \%$ respectively & \\
\hline & $\mathrm{L}$ & $\mathrm{Aq}$ & Antipyretic (In vitro) & $\begin{array}{c}\text { At } 400 \mathrm{mg} / \mathrm{kg} \text {, a moderate antipyretic activity is reported by decreasing } \\
\text { the temperature at } 36.61^{\circ} \mathrm{C}\end{array}$ & \\
\hline & $\mathrm{L}$ & $\mathrm{Aq}$ & $\begin{array}{l}\text { Cytotoxicity using micro } \\
\text { culture tetrazolium assay } \\
\text { (MTT assay) (In vitro) }\end{array}$ & Active; $\mathrm{IC}_{50}$ at $200 \mu \mathrm{g} / \mathrm{mL}=97.77$ & [107] \\
\hline & $\mathrm{L}$ & $\mathrm{Me}$ & $\begin{array}{l}\text { Thrombolytic activity } \\
\text { (In vitro) }\end{array}$ & $\begin{array}{l}\text { At dosage } 2,4,6,8 \text {, and } 10 \mathrm{mg} / \mathrm{mL}, \% \text { of clot lysis }=9.57 \pm 1.06 \%, 13.35 \\
\pm 1.67 \%, 19.35 \pm 1.84 \%, 28.23 \pm 1.97 \% \text {, and } 32.76 \pm 1.22 \% \text {, respectively }\end{array}$ & \multirow{3}{*}[45]{} \\
\hline & $\mathrm{L}$ & $\mathrm{Me}$ & $\begin{array}{l}\text { Membrane stabilizing } \\
\text { activity-Hypotonic } \\
\text { solution-induced hemolysis } \\
\text { (In vitro) }\end{array}$ & $\begin{array}{c}\text { At dosage } 2,4,6,8, \text { and } 10 \mathrm{mg} / \mathrm{mL}, \% \text { inhibition of hemolysis }=22.80 \pm \\
0.49 \%, 30.80 \pm 0.6 \%, 35.30 \pm 0.74 \%, 40.80 \pm 0.89 \% \text {, and } 45.80 \pm 0.77 \% \\
\text { respectively }\end{array}$ & \\
\hline & $\mathrm{L}$ & $\mathrm{Me}$ & $\begin{array}{l}\text { Antibacterial—Disc diffusion } \\
\text { (In vitro) }\end{array}$ & Active against $100 \mu \mathrm{L}$ of ST and EC. Inactive against SA and PA & \\
\hline \multirow{6}{*}{$\begin{array}{l}\text { Aegiceras corniculatum } \\
\text { (L.) Blanco }\end{array}$} & St & $\mathrm{H}$ & \multirow{3}{*}{ Toxicity (In vivo) } & Non-toxic at $1 \mathrm{~g} / \mathrm{kg}$ & \multirow{6}{*}[74]{} \\
\hline & St & EA & & $\mathrm{LD}_{50}(\mathrm{mg} / \mathrm{kg})=850$ & \\
\hline & St & $\mathrm{Me}$ & & Toxic above $200 \mathrm{mg} / \mathrm{kg}$ & \\
\hline & St & EA & \multirow{3}{*}{$\begin{array}{l}\text { Antinociceptive- Acetic } \\
\text { acid-induced writhings in } \\
\text { mice (In vivo) }\end{array}$} & $\begin{array}{l}\text { At } 10 \text { and } 50 \mathrm{mg} / \mathrm{kg}, \% \text { inhibition }=29 \pm 2.5 \% \text { and } 53 \pm 3.0 \% \text {, } \\
\text { respectively, } \mathrm{IC}_{50}(\mathrm{mg} / \mathrm{kg}) \text { at } 50 \mathrm{mg} / \mathrm{kg}=52 \pm 4.2\end{array}$ & \\
\hline & St & $\mathrm{H}$ & & $\begin{array}{c}\text { At } 25,50 \text {, and } 100 \mathrm{mg} / \mathrm{kg}, \% \text { inhibition }=12 \pm 0.7 \%, 28 \pm 2.5 \% \text {, } \\
\text { and } 37 \pm 3.5 \% \text {, respectively }\end{array}$ & \\
\hline & St & $\mathrm{Me}$ & & $\begin{array}{c}\text { At } 1,5 \text {, and } 10 \mathrm{mg} / \mathrm{kg}, \% \text { inhibition }=33.4 \pm 3.3 \%, 55.6 \pm 6.2 \% \text {, and } 82.4 \\
\pm 7.3 \% \text {, respectively. Me extract at } 5 \mathrm{mg} / \mathrm{kg} \text { is more potent with } \mathrm{IC}_{50} \\
\text { value of } 4.2 \pm 0.99\end{array}$ & \\
\hline
\end{tabular}


Table 7. Cont.

\begin{tabular}{|c|c|c|c|c|c|}
\hline Species & Plant Part(s) & Extract & Study/Assays & Activity & Reference \\
\hline \multirow{7}{*}{$\begin{array}{l}\text { Aegiceras corniculatum } \\
\text { (L.) Blanco }\end{array}$} & $\mathrm{AP}$ & $\mathrm{H}$ & \multirow{3}{*}{$\begin{array}{l}\text { Anti-inflammatory- } \\
\text { Carrageenan induced paw } \\
\text { edema in rats (In vivo) }\end{array}$} & $\begin{array}{l}\text { At } 10,25 \text {, and } 50 \mathrm{mg} / \mathrm{kg}, \% \text { inhibition }=15.8 \pm 2.0 \%, 39.2 \pm 3.9 \% \text {, and } \\
65.0 \pm 4.0 \% \text {, respectively }\end{array}$ & \multirow{3}{*}{ [75] } \\
\hline & $\mathrm{AP}$ & EA & & $\begin{array}{l}\text { At } 1,5 \text {, and } 10 \mathrm{mg} / \mathrm{kg}, \% \text { inhibition }=28.4 \pm 4.7 \%, 40.6 \pm 2.1 \% \text {, and } \\
51.4 \pm 2.7 \% \text {, respectively }\end{array}$ & \\
\hline & $\mathrm{AP}$ & $\mathrm{Me}$ & & At $100 \mathrm{mg} / \mathrm{kg}, \%$ inhibition $=10.8 \pm 3.4 \%$ & \\
\hline & $\mathrm{L}$ & CE & $\begin{array}{l}\text { Antibacterial using REMA } \\
\text { assay (In vitro) }\end{array}$ & $\begin{array}{l}\text { Active against BS (gram-positive) and EC (gram-negative) at } \\
\qquad 5 \mathrm{mg} / \mathrm{mL}\end{array}$ & [108] \\
\hline & $\mathrm{L}, \mathrm{Sb}, \mathrm{R}$ & $\mathrm{Me}$ & Antioxidant-FRAP (In vitro) & $\begin{array}{l}\text { AAE }(\mathrm{mg} / \mathrm{g}) \text { for the } 3 \text { methanolic extracts of each plant parts = } \\
5.31 \pm 0.11,8.18 \pm 0.14 \text {, and } 5.03 \pm 0.73 \text {, respectively }\end{array}$ & \multirow{2}{*}{ [105] } \\
\hline & $\mathrm{L}, \mathrm{Sb}, \mathrm{R}$ & $\mathrm{Me}$ & Antioxidant-DPPH (In vitro) & $\begin{array}{l}\mathrm{IC}_{50}(\mathrm{mg} / \mathrm{mL}) \text { for the } 3 \text { methanolic extracts of each plant parts }= \\
129.95 \pm 3.29,96.74 \pm 2.52, \text { and } 233.53 \pm 56.25 \text {, respectively }\end{array}$ & \\
\hline & $\mathrm{L}$ & EA & $\begin{array}{l}\text { Antimicrobial-Disc diffusion } \\
\text { assay (In vitro) }\end{array}$ & $\begin{array}{c}\text { Zone of inhibition }(\mathrm{mm}) \text { against EC, AGT, STM, and SA }=6.9 \pm 0.4 \\
8.25 \pm 0.3,6.5 \pm 0.5 \text {, and } 8.0 \pm 0.4 \text {, respectively, Inactive against } \\
\text { AF and TR }\end{array}$ & [106] \\
\hline \multirow{4}{*}{ Acrostichum aureum $\mathrm{L}$. } & $\mathrm{L}$ & $\mathrm{Me}$ & \multirow{4}{*}{$\begin{array}{l}\text { Antibacterial-Disc diffusion } \\
\text { (In vitro) }\end{array}$} & Zone of inhibition $(\mathrm{mm})$ against $\mathrm{EC}=10 \pm 0.12, \mathrm{SM}=7.6 \pm 0.58$ & \multirow{4}{*}{ [76] } \\
\hline & $\mathrm{L}$ & Ac & & $\begin{array}{l}\text { Zone of inhibition }(\mathrm{mm}) \text { against } \mathrm{PA}, \mathrm{SA}, \mathrm{EC} \text { and } \mathrm{SM}=12.3 \pm 0.23 \\
\quad 9.7 \pm 0.48,10.6 \pm 0.14 \text {, and } 7 \pm 0.32 \text {, respectively }\end{array}$ & \\
\hline & $\mathrm{L}$ & PE & & No activity observed & \\
\hline & $\mathrm{L}$ & W & & No activity observed & \\
\hline \multirow{3}{*}{$\begin{array}{l}\text { Avicennia marina } \\
\text { (Forssk.) Vierh }\end{array}$} & $\mathrm{L}$ & A & $\begin{array}{l}\text { Antimicrobial- Agar well } \\
\text { diffusion (In vitro) }\end{array}$ & Active against BC, EF, SA, SM, and AT & [7] \\
\hline & $\mathrm{L}$ & $\mathrm{E}$ & $\begin{array}{l}\text { Anti-inflammatory- Rat } \\
\text { model of rheumatoid arthritis } \\
\text { (In vivo) }\end{array}$ & $\begin{array}{l}\text { Inflammatory markers were observed to be reduced and joint lesions } \\
\text { were improved }\end{array}$ & {$[109,110]$} \\
\hline & $\mathrm{L}$ & $\mathrm{E}$ & Antiviral (In vitro) & Active against HIV, SFV, EMVC, and HBV & [98] \\
\hline
\end{tabular}


Table 7. Cont.

\begin{tabular}{|c|c|c|c|c|c|}
\hline Species & Plant Part(s) & Extract & Study/Assays & Activity & Reference \\
\hline \multirow{7}{*}{$\begin{array}{l}\text { Avicennia marina } \\
\text { (Forssk.) Vierh }\end{array}$} & $\mathrm{L}$ & $\mathrm{E}$ & $\begin{array}{l}\text { Antimutagenic- MTT assay } \\
\text { (In vitro) }\end{array}$ & $\begin{array}{l}\text { Strong effect with inhibition rates of } 68 \% \text { and } 71 \% \text { with and without } \\
\text { metabolic activation S9 }\end{array}$ & \multirow{2}{*}{ [111] } \\
\hline & $\mathrm{L}$ & $\mathrm{E}$ & $\begin{array}{l}\text { Anticancer- MTT assay } \\
\text { (In vitro) }\end{array}$ & $\begin{array}{l}\text { Significant cytotoxic effect on HL- } 60 \text { cells and induced apoptosis in } \\
\text { HL- } 60 \text { cell line }\end{array}$ & \\
\hline & NI & $\mathrm{Me}$ & Antioxidant- ABTS (In vitro) & Strong activity & [112] \\
\hline & $\mathrm{L}$ & NI & Antimicrobial (In vitro) & $\begin{array}{c}\text { Zone of inhibition (mm) against EC, SA, BS, CA, and AN }=12,6,7,9 \text {, } \\
\text { and 10, respectively for } 30 \mu \mathrm{l} \text { of extract }\end{array}$ & [113] \\
\hline & $\mathrm{L}$ & Ac & $\begin{array}{l}\text { Antimicrobial- Disc diffusion } \\
\text { assay (In vitro) }\end{array}$ & $\begin{array}{c}\text { Zone of inhibition }(\mathrm{mm}) \text { against } \mathrm{AGT}, \mathrm{STM}, \mathrm{SA} \text {, and TR are } 6.8 \pm 0.9 \\
7.5 \pm 0.5,9.1 \pm 0.3 \text {, and } 6.5 \pm 0.35, \text { respectively. Inactive against EC } \\
\text { and TR }\end{array}$ & [106] \\
\hline & $\mathrm{L}$ & $\mathrm{CE}$ & $\begin{array}{l}\text { Antimicrobial- Disc diffusion } \\
\text { assay (In vitro) }\end{array}$ & $\begin{array}{l}\text { Zone of inhibition (mm) against SA, KP, PA, BS, EC, ENA, PS, SP, and } \\
\qquad S=18,24,26,16,27,8,12,5 \text {, and 1, respectively }\end{array}$ & \multirow[t]{2}{*}{ [114] } \\
\hline & $\mathrm{L}$ & CE & Antioxidant- DPPH (In vitro) & $\%$ radical scavenging $=88.93 \%$ & \\
\hline $\begin{array}{l}\text { Avicennia germinans } \\
\text { (L.) L. }\end{array}$ & $\mathrm{L}$ & $\mathrm{Me}$ & $\begin{array}{l}\text { Antibacterial- Disc diffusion } \\
\text { assay (In vitro) }\end{array}$ & $\begin{array}{l}\text { At } 100 \mathrm{mg} \text {, zone of inhibition }(\mathrm{mm}) \text { against } \mathrm{EC}, \mathrm{KS}, \mathrm{PS} \text {, and } \mathrm{SA}=16 \text {, } \\
22,12 \text {, and } 18\end{array}$ & [77] \\
\hline \multirow{7}{*}{ Avicennia officinalis $\mathrm{L}$} & $\mathrm{L}$ & $\mathrm{E}$ & Antioxidant- DPPH (In vitro) & $\mathrm{IC}_{50}($ control $)=65.12 \pm 54, \mathrm{IC}_{50}$ (extract) at $0.1 \mathrm{mg} / \mathrm{mL}=40.77 \pm 3.43$ & \multirow{4}{*}{ [80] } \\
\hline & $\mathrm{L}$ & $\mathrm{E}$ & Antioxidant- $\mathrm{HO}$ (In vitro) & $\mathrm{IC}_{50}($ control $)=64.35 \pm 1.34, \mathrm{IC}_{50}($ extract $)=38.23 \pm 3.84$ & \\
\hline & $\mathrm{L}$ & $\mathrm{E}$ & Antioxidant- NO (In vitro) & At $0.1 \mathrm{mg} / \mathrm{mL}, \mathrm{IC}_{50}:$ control $=62.97 \pm 8.64$, extract $=39.87 \pm 4.78$ & \\
\hline & $\mathrm{L}$ & $\mathrm{E}$ & $\begin{array}{l}\text { Antioxidant- ABTS } \\
\text { (In vitro) }\end{array}$ & At $0.1 \mathrm{mg} / \mathrm{mL}, \mathrm{IC}_{50}:$ control $=61.84 \pm 1.33$, extract $=38.78 \pm 9.62$ & \\
\hline & $\mathrm{L}$ & EA & $\begin{array}{l}\text { Antimicrobial- Disc diffusion } \\
\text { assay (In vitro) }\end{array}$ & $\begin{array}{c}\text { Zone of inhibition }(\mathrm{mm}) \text { against EC, STM, and SA }=7.8 \pm 0.7,7 \pm 0.1, \\
\text { and } 7.7 \pm 0.5, \text { respectively, inactive against AF and TR }\end{array}$ & [106] \\
\hline & $\mathrm{R}$ & $\mathrm{A}, \mathrm{E}, \mathrm{Me}$ & $\begin{array}{l}\text { Antimicrobial- Agar well } \\
\text { diffusion (In vitro) }\end{array}$ & $\begin{array}{l}\text { For the three extracts activity observed with EC, SA, ENA, KP, PA, } \\
\qquad \mathrm{BS}, \mathrm{LD} \text {, and SP }\end{array}$ & [112] \\
\hline & NI & $\mathrm{E}$ & $\begin{array}{l}\text { Antiulcer- } \\
\text { Indomethacine-induced } \\
\text { gastric ulcer (In vitro) }\end{array}$ & $\begin{array}{l}\text { Gastric ulcers observed to decrease when glutathione is reduced in } \\
\text { the gastric mucosa }\end{array}$ & [115] \\
\hline
\end{tabular}


Table 7. Cont.

\begin{tabular}{|c|c|c|c|c|c|}
\hline Species & Plant Part(s) & Extract & Study/Assays & Activity & Reference \\
\hline \multirow{14}{*}{ Avicennia officinalis $\mathrm{L}$} & $\mathrm{L}$ & $\mathrm{Me}$ & $\begin{array}{l}\text { Anti-inflammatory- } \\
\text { Carrageenan induced paw } \\
\text { edema (In vivo) }\end{array}$ & $\begin{array}{l}\text { Inhibition of prostaglandin effect more potent in chronic model than } \\
\text { in acute model }\end{array}$ & [79] \\
\hline & $\mathrm{L}$ & $\mathrm{Me}$ & $\begin{array}{l}\text { Diuretic- Lipschitz dirutic } \\
\text { model (In vivo) }\end{array}$ & $\begin{array}{l}\text { At dosage } 200 \text { and } 400 \mathrm{mg} / \mathrm{kg} \text {, volume of urine }=3.06 \pm 0.18 \text { and } \\
\qquad 3.89 \pm 0.13 \mathrm{~mL} \text {, respectively }\end{array}$ & \multirow{6}{*}{ [116] } \\
\hline & $\mathrm{L}$ & $\mathrm{Me}$ & $\begin{array}{l}\text { Neuropharmacological- } \\
\text { Pentobarbital induced } \\
\text { hypnosis test (In vivo) }\end{array}$ & $\begin{array}{l}\text { At dosage } 250 \text { and } 500 \mathrm{mg} / \mathrm{kg} \text {, total sleeping time }=6.74 \pm 2.83 \text { and } \\
82.07 \pm 3.57 \mathrm{~min} \text {, respectively while with control }(0.1 \% \text { Tween } 80) \\
\text { time }=32.06 \pm 1.20 \mathrm{~min}\end{array}$ & \\
\hline & \multirow[t]{2}{*}{$\mathrm{L}$} & \multirow[t]{2}{*}{$\mathrm{Me}$} & \multirow[t]{2}{*}{$\begin{array}{l}\text { Neuropharmacological- Open } \\
\text { field test (In vivo) }\end{array}$} & $\begin{array}{l}\text { At dosage } 250 \mathrm{mg} / \mathrm{kg} \text {, number of movements before and after drug } \\
\text { administration after } 90 \mathrm{~min}=110.50 \pm 2.12 \text { and } 41.85 \pm 3.35 \\
\text { respectively }\end{array}$ & \\
\hline & & & & $\begin{array}{l}\text { At dosage } 500 \mathrm{mg} / \mathrm{kg}, \text { number of movements before and after drug } \\
\text { administration after } 90 \mathrm{~min}=107.99 \pm 2.70 \text { and } 30.06 \pm 2.64 \\
\text { respectively }\end{array}$ & \\
\hline & \multirow[t]{2}{*}{$\mathrm{L}$} & \multirow[t]{2}{*}{$\mathrm{Me}$} & \multirow{2}{*}{$\begin{array}{l}\text { Neuropharmacological- Hole } \\
\text { cross test (In vivo) }\end{array}$} & $\begin{array}{l}\text { At dosage } 250 \mathrm{mg} / \mathrm{kg} \text {, number of holes crossed before and after drug } \\
\text { administration after } 90 \mathrm{~min}=7.57 \pm 0.18 \text { and } 5.30 \pm 0.69 \text {, respectively }\end{array}$ & \\
\hline & & & & $\begin{array}{l}\text { At dosage } 500 \mathrm{mg} / \mathrm{kg} \text {, number of movements before and after drug } \\
\text { administration after } 90 \mathrm{~min}=6.61 \pm 0.72 \text { and } 4.90 \pm 0.67 \text {, respectively }\end{array}$ & \\
\hline & $\mathrm{L}$ & PE & \multirow{2}{*}{$\begin{array}{l}\text { Anti-HIV- Reverse } \\
\text { transcriptase (RT) inhibition } \\
\text { assay (In vitro) }\end{array}$} & \%inhibition: control = $71.04 \pm 1.94$, extract $=74.79 \pm 3.47$ & \multirow{2}{*}[117]{} \\
\hline & $\mathrm{L}$ & E & & \%inhibition: control $(\mathrm{AZT})=71.04 \pm 1.94$, extract $=82.00 \pm 0.26$ & \\
\hline & Fr & $\mathrm{E}$ & Antioxidant- ABTS (In vitro) & Activity highest with ABTS compared to DPPH and FRAP & [112] \\
\hline & $\mathrm{L}$ & $\mathrm{E}$ & Toxicity (In vivo) & $\begin{array}{l}\text { No significant change observed in the majority of the mice. Mortality } \\
\text { rate was zero }\end{array}$ & [115] \\
\hline & $\mathrm{L}$ & $\mathrm{E}$ & Antioxidant- DPPH (In vitro) & $\begin{array}{l}\text { At dosage } 10 \text { and } 100 \mu \mathrm{g} / \mathrm{mL}, \% \text { inhibition }=16.34 \% \text { and } 63.64 \% \text {, } \\
\text { respectively }\end{array}$ & \multirow{3}{*}{ [118] } \\
\hline & $\mathrm{L}$ & $\mathrm{E}$ & Cytotoxic (In vitro) & $\mathrm{LC}_{50}(\mu \mathrm{g} / \mathrm{mL})=131.2$ & \\
\hline & $\mathrm{L}$ & E & $\begin{array}{l}\text { Antibacterial- Disc diffusion } \\
\text { (In vitro) }\end{array}$ & Active against EC and ST, MIC $(\mu \mathrm{g} / \mathrm{mL})$ against $\mathrm{EC}=62.5, \mathrm{ST}=125$ & \\
\hline
\end{tabular}


Table 7. Cont

\begin{tabular}{|c|c|c|c|c|c|}
\hline Species & Plant Part(s) & Extract & Study/Assays & Activity & Reference \\
\hline \multirow[t]{2}{*}{$\begin{array}{l}\text { Bruguiera cylindrica }(\mathrm{L} .) \\
\text { Blume }\end{array}$} & St & $\begin{array}{c}\mathrm{Bu}, \mathrm{C}, \mathrm{E}, \\
\mathrm{H}, \mathrm{Aq}\end{array}$ & $\begin{array}{l}\text { Antioxidant- Oxygen free } \\
\text { radical generation (In vitro) }\end{array}$ & $\begin{array}{l}\text { \%inhibition for all extracts ranged from } 18-77 \text { for superoxide anions } \\
\left(\mathrm{O}^{2-}\right), 29-43 \text { for hydroxyl radical }\left(\mathrm{OH}^{\bullet}\right) \text { and } 20-39 \text { for } \\
\text { microsomallipid peroxidation }\end{array}$ & [119] \\
\hline & $\mathrm{L}, \mathrm{St}$ & $\mathrm{Me}$ & Antioxidant- DPPH (In vitro) & $\mathrm{IC}_{50}(\mu \mathrm{g} / \mathrm{mL})$ for $\mathrm{L}=175, \mathrm{St}=162.5$ & [120] \\
\hline \multirow{13}{*}{$\begin{array}{l}\text { Brugueira gymnorhiza } \\
\text { (L.) Lam }\end{array}$} & $\mathrm{L}$ & $\mathrm{Me}$ & $\begin{array}{l}\text { Antinociceptive- Acetic } \\
\text { acid-induced writhing in mice } \\
\text { (In vivo) }\end{array}$ & $\begin{array}{c}\text { At dosage } 250 \text { and } 500 \mathrm{mg} / \mathrm{kg}, \% \text { writhing inhibition }=46 \% \text { and } 59 \% \text {, } \\
\text { respectively. Control }(25 \mathrm{mg} / \mathrm{kg})=63 \%\end{array}$ & \multirow{2}{*}[81]{} \\
\hline & $\mathrm{L}$ & $\mathrm{Me}$ & Anti-diarrheal (In vivo) & $\begin{array}{l}\text { Latent period (h) for control (loperamide) and at dosage } 500 \mathrm{mg}= \\
1.71 \pm 0.145 \text { and } 1.67 \pm 0.163 \text {, respectively }\end{array}$ & \\
\hline & $\mathrm{L}$ & $\mathrm{CE}$ & $\begin{array}{l}\text { Anti-inflammatory- COX } \\
\text { inhibition assay (In vitro) }\end{array}$ & $\begin{array}{c}\% \text { inhibition at } 10 \text { and } 100 \mu \mathrm{g} / \mathrm{mL}=9.7 \pm 7.2 \text { and } 65.1 \pm 5.8 \\
\text { respectively }\end{array}$ & \multirow[t]{5}{*}{ [121] } \\
\hline & $\mathrm{L}$ & $\mathrm{CE}$ & Antioxidant- DPPH (In vitro) & $\%$ inhibition at 2 and $1 \mathrm{mg} / \mathrm{mL}=68 \%$ and $59 \%$, respectively & \\
\hline & $\mathrm{B}$ & $\mathrm{C}, \mathrm{E}, \mathrm{Me}$ & Antioxidant- DPPH (In vitro) & $\mathrm{IC}_{50}: \mathrm{C}=0.27 \pm 0.017, \mathrm{E}=0.029 \pm 0.004, \mathrm{Me}=0.038 \pm 0.003$ & \\
\hline & $\mathrm{L}$ & $\mathrm{Me}$ & Antimicrobial (In vitro) & $\begin{array}{c}\text { Zone of inhibition }(\mathrm{mm}) \text { against } \mathrm{BC}, \mathrm{SA}, \mathrm{EC} \text {, and PA are } 12.67,14.34 \text {, } \\
8.87 \text {, and } 7.85 \text {, respectively }\end{array}$ & \\
\hline & B & $\mathrm{Me}$ & Toxicity (In vivo) & $\begin{array}{c}\text { Zone of inhibition (mm) against BC, SA, EC, and PA are 15.86, 17.85, } \\
9.25 \text {, and } 8.38 \text {, respectively }\end{array}$ & \\
\hline & $\mathrm{R}$ & $\mathrm{E}$ & & $\begin{array}{l}\text { Non-toxic, no significant change in behavior or neurological } \\
\text { response up to } 400 \mathrm{mg} / \mathrm{kg} \text { body weight }\end{array}$ & \multirow{4}{*}{ [82] } \\
\hline & \multirow{3}{*}{$\mathrm{R}$} & \multirow{3}{*}{$\mathrm{E}$} & \multirow{3}{*}{$\begin{array}{l}\text { Antihyperglycemic- STZ } \\
\text { induced diabetic rats (In vivo) }\end{array}$} & $\begin{array}{l}\text { Serum glucose levels of control and extract }(400 \mathrm{mg} / \mathrm{kg}) \text { at day } 0= \\
224.70 \pm 15.52 \text { and } 237.0 \pm 15.0 \mathrm{mg} / \mathrm{mL} \text {, respectively }\end{array}$ & \\
\hline & & & & $\begin{array}{l}\text { Serum glucose levels of control and extract }(400 \mathrm{mg} / \mathrm{kg}) \text { at day } 7= \\
214.5 \pm 2.60 \text { and } 188.10 \pm 3.14 \mathrm{mg} / \mathrm{mL} \text {, respectively }\end{array}$ & \\
\hline & & & & $\begin{array}{c}\text { Serum glucose levels of control and extract }(400 \mathrm{mg} / \mathrm{kg}) \text { at day } 28= \\
201 \pm 16.32 \text { and } 89.04 \pm 10.23 \mathrm{mg} / \mathrm{mL} \text {, respectively. A significant } \\
\text { decrease is observed in the blood glucose level compared to diabetic } \\
\text { control rats }\end{array}$ & \\
\hline & $\mathrm{L}$ & $\mathrm{Me}$ & Antimicrobial (In vitro) & Zone of inhibition $(\mathrm{mm})$ against $\mathrm{EC}=22$ & \multirow[b]{2}{*}{ [62] } \\
\hline & $\mathrm{B}$ & $\mathrm{H}$ & & $\begin{array}{l}\text { Zone of inhibition (mm) against KP, ST, SA and SF are 23, 22, } 19 \text { and } \\
22 \text { respectively }\end{array}$ & \\
\hline
\end{tabular}


Table 7. Cont.

\begin{tabular}{|c|c|c|c|c|c|}
\hline Species & Plant Part(s) & Extract & Study/Assays & Activity & Reference \\
\hline & $\mathrm{L}$ & $\mathrm{Me}$ & Antioxidant (In vitro) & $\begin{array}{c}\mathrm{IC}_{50}(\mu \mathrm{g} / \mathrm{mL}) \text { for FRAP, DPPH, NO, SO, HO and ABTS radical } \\
\text { scavenging }=17.93 \pm 0.161,0.355 \pm 0.005,0.305 \pm 0.004,0.356 \pm 0.007 \\
0.311 \pm 0.004 \text { and } 0.056 \pm 0.0003 \text { respectively }\end{array}$ & \\
\hline & $\mathrm{L}$ & $\mathrm{Me}$ & $\begin{array}{l}\text { Hepatoprotective- GaIN } \\
\text { induced hepatic toxicity in } \\
\text { rats (In vivo) }\end{array}$ & $\begin{array}{l}\text { With sample GaIN }+ \text { extract }(125 \mathrm{mg} / \mathrm{kg}), \mathrm{ALT}, \mathrm{AST}, \mathrm{AKP}, \text { and total } \\
\text { protein were exhibited to be } 76.6 \pm 2.75,79.3 \pm 2.49,121 \pm 3.19, \text { and } 4.46 \\
\pm 0.12 . \text { With sample GaIN }+ \text { extract }(250 \mathrm{mg} / \mathrm{kg}), \mathrm{ALT}, \mathrm{AST}, \mathrm{AKP} \text {, and } \\
\text { total protein were exhibited to be } 68.8 \pm 2.27,69.1 \pm 1.66,108.8 \pm 3.43 \\
\text { and } 5.01 \pm 0.11\end{array}$ & {$[64]$} \\
\hline & $\mathrm{L}, \mathrm{Sb}, \mathrm{R}$ & $\mathrm{Me}$ & Antioxidant- FRAP (In vitro) & $\begin{array}{c}\text { AAE }(\mathrm{mg} / \mathrm{g}) \text { for the } 3 \text { methanolic extracts of each plant parts }=1.25 \pm \\
0.03,2.85 \pm 0.09 \text {, and } 1.55 \pm 0.16 \text {, respectively }\end{array}$ & \\
\hline & $\mathrm{L}, \mathrm{Sb}, \mathrm{R}$ & $\mathrm{Me}$ & Antioxidant- DPPH (In vitro) & $\begin{array}{c}\mathrm{IC}_{50}(\mathrm{mg} / \mathrm{g}) \text { for the } 3 \text { methanolic extracts of each plant parts }=2052.20 \pm \\
172.01,254.69 \pm 21.26 \text {, and } 1532.71 \pm 46.32, \text { respectively }\end{array}$ & {$[105,122]$} \\
\hline & $\mathrm{NI}$ & $\mathrm{Me}$ & Cytotoxicity (In vivo) & $\mathrm{IC}_{50}>2.5 \mathrm{mg} / \mathrm{mL}$ & \\
\hline \multirow{3}{*}{$\begin{array}{l}\text { Bruguiera parviflora } \\
\text { (Roxb.) Wight \& Arn. } \\
\text { ex Griff }\end{array}$} & $\mathrm{L}$ & EA & Antioxidant- DPPH (In vitro) & $\mathrm{EC}_{50}(\mu \mathrm{g} / \mathrm{mL})=105.00$ & \multirow{3}{*}{ [123] } \\
\hline & $\mathrm{L}$ & EA & $\begin{array}{l}\text { Antioxidant- Lipid } \\
\text { peroxidation inhibition } \\
\text { (In vitro) }\end{array}$ & $\mathrm{IC}_{50}(\mu \mathrm{g} / \mathrm{mL})=42.60$ & \\
\hline & $\mathrm{L}$ & EA & $\begin{array}{l}\text { Antioxidant- Quinone } \\
\text { reductase induction activity } \\
\text { (In vitro) }\end{array}$ & $\mathrm{CD}(\mu \mathrm{g} / \mathrm{mL})>10, \mathrm{IC}_{50}(\mu \mathrm{g} / \mathrm{mL})>20$ & \\
\hline $\begin{array}{l}\text { Bruguiera sexangula } \\
\text { (Lour.) Poir. }\end{array}$ & $\mathrm{L}$ & EA & $\begin{array}{l}\text { Antibacterial- Agar diffusion } \\
\text { (In vitro) }\end{array}$ & Inhibition against SA and PS & [124] \\
\hline \multirow{5}{*}{$\begin{array}{l}\text { Ceriops decandra (Griff.) } \\
\text { W. Theob. }\end{array}$} & $\mathrm{L}, \mathrm{Sb}, \mathrm{R}$ & $\mathrm{Me}$ & Antioxidant- FRAP (In vitro) & $\begin{array}{l}\text { AAE }(\mathrm{mg} / \mathrm{g}) \text { for the } 3 \text { methanolic extracts of each plant parts }=0.90 \pm \\
0.66,13.04 \pm 0.75 \text { and } 9.81 \pm 0.87 \text { respectively }\end{array}$ & \multirow{2}{*}{ [105] } \\
\hline & $\mathrm{L}, \mathrm{Sb}, \mathrm{R}$ & $\mathrm{Me}$ & Antioxidant- DPPH (In vitro) & $\begin{array}{l}\mathrm{IC}_{50}(\mathrm{mg} / \mathrm{g}) \text { for the } 3 \text { methanolic extracts of each plant parts }=5666.86 \pm \\
324.46,65.55 \pm 1.35 \text {, and } 93.65 \pm 3.52 \text {, respectively }\end{array}$ & \\
\hline & B & $\mathrm{E}$ & $\begin{array}{l}\text { Anti-inflammatory- } \\
\text { Carrageenan-induced paw } \\
\text { edema test (In vivo) }\end{array}$ & $\begin{array}{c}\text { \%inhibition of extract }(400 \mathrm{mg} / \mathrm{kg})=67.72 \text { while that of standard drug, } \\
\text { indomethacin is } 69.29 \%\end{array}$ & \multirow[t]{2}{*}{ [125] } \\
\hline & B & $\mathrm{E}$ & Antioxidant- DPPH (In vitro) & $\mathrm{IC}_{50}(\mu \mathrm{g} / \mathrm{mL})=12.90$ & \\
\hline & $\mathrm{L}$ & EA & $\begin{array}{l}\text { Antimicrobial- Disc diffusion } \\
\text { assay (In vitro) }\end{array}$ & $\begin{array}{l}\text { Zone of inhibition }(\mathrm{mm}) \text { against EC, AGT, STM, and SA are } 8.3 \pm 0.5,9.0 \\
\pm 0.8,7.8 \pm 0.2 \text {, and } 8.5 \pm 0.45 \text {, respectively, Inactive against AF and TR }\end{array}$ & [106] \\
\hline
\end{tabular}


Table 7. Cont.

\begin{tabular}{|c|c|c|c|c|c|}
\hline Species & Plant Part(s) & Extract & Study/Assays & Activity & Reference \\
\hline $\begin{array}{l}\text { Ceriops roxburghiana } \\
\text { Arn. }\end{array}$ & $\mathrm{L}$ & NI & $\begin{array}{l}\text { Anti-HIV- MTT assay } \\
\text { (In vitro) }\end{array}$ & $\begin{array}{c}\mathrm{CC}_{50}(\mu \mathrm{g} / \mathrm{mL})=216.54 \pm 14.21, \mathrm{EC}_{50}(\mu \mathrm{g} / \mathrm{mL})=13.38 \pm 3.15 \\
\mathrm{SI}=16.18\end{array}$ & [87] \\
\hline \multirow{7}{*}{ Excoecariaa gallocha $\mathrm{L}$. } & $\mathrm{L}$ & $\mathrm{Me}$ & Antioxidant (In vitro) & $\begin{array}{c}\mathrm{IC}_{50}(\mu \mathrm{g} / \mu \mathrm{l}): \mathrm{DPPH}=67.50, \mathrm{NO} \text { inhibition }=4.8, \text { lipid peroxidation } \\
\text { inhibition }=100, \text { metal chelating } \operatorname{effect}(\mu \mathrm{g})=2.47\end{array}$ & \multirow{6}{*}[50]{} \\
\hline & La, L, S & $\mathrm{E}$ & $\begin{array}{l}\text { Anti-inflammatory- } \\
\text { Carrageenan-induced paw } \\
\text { edema test (In vivo) }\end{array}$ & $\begin{array}{l}\% \text { inhibition at } 500 \mathrm{mg} / \mathrm{kg} \text { for all } 3 \text { extracts are } 63.15 \%, 62.15 \% \text {, and } \\
69.29 \% \text {, respectively }\end{array}$ & \\
\hline & $\mathrm{S}$ & NI & $\begin{array}{l}\text { Anti-inflammatory- } \\
\text { Pellet-induced granuloma test } \\
\text { (In vivo) }\end{array}$ & $\begin{array}{l}\text { At dosage } 500 \mathrm{mg} / \mathrm{kg} \text {, activity was highest with a \%reduction of } \\
57.03 \% \text {. }\end{array}$ & \\
\hline & B & $\mathrm{E}$ & $\begin{array}{l}\text { Analgesic- Acetic } \\
\text { acid-induced writhing test in } \\
\text { mice (In vivo) }\end{array}$ & $\begin{array}{l}\text { At dosage } 500 \mathrm{mg} / \mathrm{kg} \text {, activity was highest with a \%reduction of } \\
53.87 \%\end{array}$ & \\
\hline & St & $\mathrm{E}$ & $\begin{array}{l}\text { Anticancer- MTS assay } \\
\text { (In vitro) }\end{array}$ & $\begin{array}{c}\mathrm{IC}_{50}(\mu \mathrm{g} / \mathrm{mL})=4 \text { and } 7 \text {, strong activity against pancreatic cancer cell } \\
\text { lines Capan- } 1 \text { and Miapaca- } 2\end{array}$ & \\
\hline & $\mathrm{L}$ & $\mathrm{Me}$ & Antifilarial (In vitro) & $\begin{array}{l}\text { Significant activity against metazoan filarial parasite Setariadigitata. } \\
\text { After } 24 \mathrm{~h} \text { treatment with extracts at a concentration of } 10,50 \text {, and } 100 \\
\mu \mathrm{g} / \mathrm{mL} \text {, developmental stages of parasite were found dead with } 30 \% \text {, } \\
\qquad 75 \%, 100 \% \text {, respectively }\end{array}$ & \\
\hline & $\mathrm{L}$ & EA & $\begin{array}{l}\text { Antimicrobial- Disc diffusion } \\
\text { assay (In vitro) }\end{array}$ & $\begin{array}{c}\text { Zone of inhibition (mm) against EC, AGT, STM, and SA }=10.3 \pm 2.7 \\
6.2 \pm 0.8,8.3 \pm 1.2, \text { and } 8.5 \pm 0.7 \text {, respectively. Inactive against AF } \\
\text { and TR }\end{array}$ & [106] \\
\hline \multirow[t]{2}{*}{$\begin{array}{l}\text { Heritiera fomes } \\
\text { Buch.-Ham }\end{array}$} & B & $\mathrm{Me}$ & $\begin{array}{l}\text { Antihyperglycemic- Oral } \\
\text { glucose tolerance test in } \\
\text { glucose-induced Swiss albino } \\
\text { mice (In vivo) }\end{array}$ & $\begin{array}{l}\text { After } 60 \mathrm{~min} \text { of glucose loading, serum glucose level with standard } \\
\text { drug (glibenclamide- } 10 \mathrm{mg} / \mathrm{kg} \text { ) and extract }(250 \mathrm{mg} / \mathrm{kg} \text { ) were } 43.5 \\
\text { and } 49.2 \text {, respectively. After } 120 \mathrm{~min} \text { of glucose loading, serum } \\
\text { glucose level with standard drug, extracts at } 250 \text { and } 500 \mathrm{mg} / \mathrm{kg} \text { were } \\
\text { 30.1,35.6, and } 44.7 \text { respectively }\end{array}$ & \multirow[t]{2}{*}{ [91] } \\
\hline & B & $\mathrm{Me}$ & $\begin{array}{l}\text { Antinociceptive- Acetic } \\
\text { acid-induced writhing in mice } \\
\text { (In vivo) }\end{array}$ & $\begin{array}{c}\text { At dosage } 100,250 \text {, and } 500 \mathrm{mg} / \mathrm{kg}, \% \text { inhibition }=8.5,26.4 \text {, and } 43.4 \text {, } \\
\text { respectively }\end{array}$ & \\
\hline
\end{tabular}


Table 7. Cont.

\begin{tabular}{|c|c|c|c|c|c|}
\hline Species & Plant Part(s) & Extract & Study/Assays & Activity & Reference \\
\hline \multirow{11}{*}{$\begin{array}{l}\text { Heritiera fomes } \\
\text { Buch.-Ham }\end{array}$} & $\mathrm{L}$ & $\mathrm{E}$ & Antioxidant- DPPH (In vitro) & $\mathrm{IC}_{50}(\mu \mathrm{g} / \mathrm{mL})=26.30$ & \multirow{3}{*}{ [89] } \\
\hline & $\mathrm{L}$ & $\mathrm{E}$ & $\begin{array}{l}\text { Antinociceptive- Acetic } \\
\text { acid-induced writhing test } \\
\text { (In vivo) }\end{array}$ & $\begin{array}{l}\text { At dosage } 250 \text { and } 500 \mathrm{mg} / \mathrm{kg} \text {, \% writhing inhibition }=34.83 \text { and } \\
59.20 \text {, respectively }\end{array}$ & \\
\hline & $\mathrm{L}$ & $\mathrm{E}$ & $\begin{array}{l}\text { Antimicrobial- Disc diffusion } \\
\text { assay (In vitro) }\end{array}$ & $\begin{array}{l}\text { Zone of inhibition }(\mathrm{mm}) \text { against } \mathrm{EC}, \mathrm{ST}, \mathrm{SP}, \mathrm{SD} \text {, and } \mathrm{SA}=3.92,7.63 \text {, } \\
5.21,7.54 \text {, and } 6.41 \text { respectively }\end{array}$ & \\
\hline & B & NI & Antidiabetic (In vitro) & $\begin{array}{l}\text { After } 60 \mathrm{~min} \text { of glucose loading at dosage } 250 \mathrm{mg} / \mathrm{kg} \text {, serum glucose } \\
\text { level was } 49.2 \text {. After } 120 \mathrm{~min} \text {, serum glucose level of extracts ( } 250 \\
\text { and } 500 \mathrm{mg} / \mathrm{kg} \text { ) and standard drug (glibenclamide) were reduced by } \\
\text { 35.6, 44.7, and 30.1, respectively }\end{array}$ & \multirow{5}{*}{ [51] } \\
\hline & $\mathrm{L}$ & NI & Antioxidant- DPPH (In vitro) & $\mathrm{IC}_{50}(\mu \mathrm{g} / \mathrm{mL})=26.30$ & \\
\hline & B & NI & Antioxidant- DPPH (In vitro) & $\mathrm{IC}_{50}(\mu \mathrm{g} / \mathrm{mL})=22, \mathrm{EC}_{50}(\mu \mathrm{g} / \mathrm{mL})=19.4$ & \\
\hline & $\mathrm{B}$ & NI & $\begin{array}{l}\text { Antinociceptive- Acetic } \\
\text { acid-induced writhing in mice } \\
\text { (In vivo) }\end{array}$ & $\begin{array}{l}\text { At dosage } 100,250 \text {, and } 500 \mathrm{mg} / \mathrm{kg} \text {, \%writhing inhibitions }=8.5 \% \text {, } \\
26.4 \% \text {, and } 43.3 \% \text {, respectively }\end{array}$ & \\
\hline & $\mathrm{L}$ & NI & & $\begin{array}{l}\text { At dosage } 250 \text { and } 500 \mathrm{mg} / \mathrm{kg} \text {, \%writhing inhibitions }=34.83 \% \text { and } \\
59.20 \% \text {, respectively }\end{array}$ & \\
\hline & $\mathrm{L}$ & $\mathrm{C}$ & Toxicity (In vitro) & $\mathrm{LC}_{50}(\mathrm{mg} / \mathrm{mL})=234.77 \pm 0.144$ & \multirow{2}{*}[69]{} \\
\hline & B & $\mathrm{Me}$ & & $\mathrm{LC}_{50}(\mathrm{mg} / \mathrm{mL})=47.081 \pm 0.056$ & \\
\hline & $\mathrm{L}$ & NI & Antioxidant-DPPH (In vitro) & $\mathrm{IC}_{50}(\mu \mathrm{g} / \mathrm{mL})=13$ & [109] \\
\hline \multirow{3}{*}{ Heritiera littoralis Aiton } & $\mathrm{L}, \mathrm{R}$ & NI & Antioxidant- DPPH (In vitro) & $\mathrm{IC}_{50}(\mathrm{mg} / \mathrm{mL}): \mathrm{L}=0.028, \mathrm{R}=0.023$ & \multirow{3}{*}{ [126] } \\
\hline & $\mathrm{L}, \mathrm{R}$ & NI & Antioxidant- HO (In vitro) & $\mathrm{IC}_{50}(\mathrm{mg} / \mathrm{mL}): \mathrm{L}=0.600, \mathrm{R}=0.536$ & \\
\hline & $\mathrm{L}, \mathrm{R}$ & NI & Antioxidant- SO (In vitro) & $\mathrm{IC}_{50}(\mathrm{mg} / \mathrm{mL}): \mathrm{L}=0.606, \mathrm{R}=0.802$ & \\
\hline \multirow{2}{*}{$\begin{array}{l}\text { Kandelia candel (L.) } \\
\text { Druce }\end{array}$} & Hy & $\begin{array}{l}\text { EA, PE, } \\
\mathrm{Aq}\end{array}$ & Antioxidant- DPPH (In vitro) & $\begin{array}{c}\mathrm{IC}_{50}(\mu \mathrm{g} / \mathrm{mL}): \mathrm{EA}=124.19 \pm 3.02, \mathrm{PE}=153.48 \pm 3.22 \\
\mathrm{~W}=132.04 \pm 2.16\end{array}$ & \multirow[b]{2}{*}[93]{} \\
\hline & Hy & $\begin{array}{l}\text { EA, PE, } \\
\text { Aq }\end{array}$ & Antioxidant- FRAP (In vitro) & $\mathrm{AAE}(\mathrm{mmol} / \mathrm{g}): \mathrm{EA}=4.39 \pm 3.17, \mathrm{PE}=2.99 \pm 0.27, \mathrm{~W}=3.69 \pm 0.04$ & \\
\hline
\end{tabular}


Table 7. Cont.

\begin{tabular}{|c|c|c|c|c|c|}
\hline Species & Plant Part(s) & Extract & Study/Assays & Activity & Reference \\
\hline \multirow{4}{*}{$\begin{array}{l}\text { Lumnitzera racemosa } \\
\text { Willd. }\end{array}$} & $\mathrm{L}$ & $\mathrm{Aq}$ & Antioxidant- DPPH (In vitro) & $\mathrm{IC}_{50}(\mu \mathrm{g} / \mathrm{mL})=38.89$ & \multirow{4}{*}{ [127] } \\
\hline & $\mathrm{L}$ & $\mathrm{Aq}$ & Antioxidant- ABTS (In vitro) & $\mathrm{IC}_{50}(\mu \mathrm{g} / \mathrm{mL})=44.38$ & \\
\hline & $\mathrm{L}$ & $\mathrm{Aq}$ & $\begin{array}{l}\text { Cytotoxicity against Hep G2 } \\
\text { cancer cell line using MTT } \\
\text { assay (In vitro) }\end{array}$ & $\begin{array}{c}\mathrm{IC}_{50}(\mu \mathrm{g} / \mathrm{mL})=26.05 ; \text { exhibited potent cytotoxicity activity on Hep } \\
\text { G2 cell lines at different concentrations }\end{array}$ & \\
\hline & $\mathrm{L}$ & $\mathrm{Aq}$ & $\begin{array}{l}\text { Anticoagulant- APTT and PT } \\
\text { assays (In vitro) }\end{array}$ & $\begin{array}{c}\text { Clotting time ratio at concentration } 100,500 \text {, and } 1000 \mu \mathrm{g} / \mathrm{mL} \text { for } \\
\text { APTT assay are } 1.2,1.4 \text {, and } 1.6, \text { respectively. Clotting time ratio at } \\
\text { concentration } 100,500 \text {, and } 1000 \mu \mathrm{g} / \mathrm{mL} \text { for PT assay are } 1.25,1.31, \\
\text { and } 1.34 \text {, respectively. Prolongation of APTT is slightly higher than } \\
\text { that of the PT assay }\end{array}$ & \\
\hline \multirow{3}{*}{ Nypa fruticans Wurmb } & NI & EA & Antioxidant- DPPH (In vitro) & $\mathrm{IC}_{50}(\mathrm{mg} / \mathrm{mL})=2.770 \pm 0.012$ & \multirow[b]{2}{*}{ [96] } \\
\hline & NI & $\mathrm{Aq}$ & $\begin{array}{l}\text { Antidiabetic- Intraperitoneal } \\
\text { glusoce tolerance test } \\
\text { (In vivo) }\end{array}$ & Blood glucose lowering effect $=56.6 \%$, serum insulin level $=79.8 \%$ & \\
\hline & $\mathrm{L}$ & $\mathrm{Me}$ & $\begin{array}{l}\text { Antimicrobial- Disc diffusion } \\
\text { assay (In vitro) }\end{array}$ & $\begin{array}{l}\text { Zone of inhibition }(\mathrm{mm}) \text { against } \mathrm{EC}, \mathrm{AGT}, \mathrm{STM} \text {, and } \mathrm{SA}=6.5 \pm 0.4 \\
7.3 \pm 0.5,6.25 \pm 0.3, \text { and } 6.8 \pm 0.3, \text { respectively. Inactive against } \mathrm{AF} \\
\text { and TR }\end{array}$ & [106] \\
\hline \multirow{2}{*}{$\begin{array}{l}\text { Pelliciera rhizophorae } \\
\text { Planch. \& Triana }\end{array}$} & $\mathrm{L}$ & NI & Antiparasitic (In vitro) & $\begin{array}{l}\text { At } 10 \mu \mathrm{g} / \mathrm{mL}, \mathrm{IC}_{50}(\mu \mathrm{M}) \text { for } \mathrm{LD}, \mathrm{PF} \text {, and } \mathrm{TC}=12.6 \pm 0.2,9.7 \pm 0.3 \text {, and } \\
13.0 \pm 0.4 \text {, respectively. Inactive against VC }\end{array}$ & \multirow{2}{*}[44]{} \\
\hline & $\mathrm{L}$ & NI & $\begin{array}{l}\text { Antidiabetic- } \alpha \text {-glucosidase } \\
\text { inhibition (In vitro) }\end{array}$ & $\begin{array}{l}\text { More potent against - } \alpha \text {-glucosidase than acarbose (positive control) } \\
\text { with } \mathrm{IC}_{50}(\mu \mathrm{M})=217.7\end{array}$ & \\
\hline Rhizophora conjugata L. & NI & $\mathrm{CE}$ & $\begin{array}{l}\text { Antimicrobial- Agar well } \\
\text { diffusion (In vitro) }\end{array}$ & $\begin{array}{c}\text { Zone of inhibition (mm) against AS, AF, CA, STM, STS, SA, and LA = } \\
7,8,11,15,19,11 \text {, and 22, respectively. Activity against LA was } \\
\text { highest }\end{array}$ & [128] \\
\hline
\end{tabular}


Table 7. Cont

\begin{tabular}{|c|c|c|c|c|c|}
\hline Species & Plant Part(s) & Extract & Study/Assays & Activity & Reference \\
\hline \multirow{6}{*}{ Rhizophora mangle L. } & B & $\mathrm{Aq}$ & $\begin{array}{l}\text { Anti ulcer- } \\
\text { Indomethacine-induced } \\
\text { gastric ulcer (In vivo) }\end{array}$ & $\begin{array}{c}\text { At dosage } 50,125,250,500, \text { and } 750 \mathrm{mg} / \mathrm{kg} \text {, the lesion indices }=5.2 \pm \\
0.84,4.5 \pm 0.58,3.25 \pm 1.71,1.6 \pm 1.95, \text { and } 4.6 \pm 0.55, \text { respectively. } \\
\text { Lesion index (control-distilled water) }=4.8 \pm 0.45\end{array}$ & [129] \\
\hline & B & $\mathrm{Aq}$ & Antioxidant- DPPH (In vitro) & $\begin{array}{l}\text { Significant decrease at } 250 \text { and } 500 \mathrm{mg} / \mathrm{kg} \text { compared to the control in } \\
\text { gastric volume }\end{array}$ & \multirow{3}{*}{ [130] } \\
\hline & \multirow{2}{*}{$\mathrm{L}$} & \multirow{2}{*}{ NI } & \multirow{2}{*}{ Antioxidant- SO (In vitro) } & $\begin{array}{c}\mathrm{IC}_{50}(\mu \mathrm{g} / \mathrm{mL}) \text { of extract and polyphenolic fraction }=6.7 \text { and } 7.6, \\
\text { respectively }\end{array}$ & \\
\hline & & & & $\begin{array}{l}\mathrm{IC}_{50}(\mu \mathrm{g} / \mathrm{mL}) \text { of extract and polyphenolic fraction }=31.9 \text { and } 21.6 \text {, } \\
\text { respectively. Activity increased as tannins concentration increased }\end{array}$ & \\
\hline & \multirow{2}{*}{$\mathrm{L}$} & \multirow{2}{*}{ NI } & Antioxidant- DPPH (In vitro) & $\mathrm{IC}_{50}(\mu \mathrm{g} / \mathrm{mL})=89.83 \pm 4.91$ & \multirow{2}{*}{ [131] } \\
\hline & & & Antioxidant- FRAP (In vitro) & $\operatorname{AAE}(\mathrm{mmol} / \mathrm{g})=12.98 \pm 1.20$ & \\
\hline \multirow{8}{*}{$\begin{array}{l}\text { Rhizophora apiculata } \\
\text { Blume }\end{array}$} & $\mathrm{R}$ & NI & Antioxidant- DPPH (In vitro) & $\mathrm{IC}_{50}(\mu \mathrm{g} / \mathrm{mL})=17$ & [109] \\
\hline & St & $\begin{array}{l}\mathrm{Bu}, \mathrm{E}, \mathrm{EE} \\
\mathrm{Aq}\end{array}$ & Antioxidant- DPPH (In vitro) & $\begin{array}{c}\mathrm{IC}_{50}(\mu \mathrm{g} / \mathrm{mL}): \mathrm{Bu}=9.68 \pm 1.86, \mathrm{E}=19.31 \pm 1.56, \mathrm{EE}=13.56 \pm 1.79 \\
\mathrm{~W}=23.72 \pm 1.94, \text { control }(\mathrm{BHT})=52.20 \pm 1.57\end{array}$ & \multirow{3}{*}{ [132] } \\
\hline & St & $\begin{array}{l}\mathrm{Bu}, \mathrm{E}, \mathrm{EE} \\
\mathrm{Aq}\end{array}$ & Antioxidant- ABTS (In vitro) & $\begin{array}{c}\mathrm{IC}_{50}(\mu \mathrm{g} / \mathrm{mL}): \mathrm{Bu}=1.26 \pm 0.05, \mathrm{E}=3.01 \pm 0.75, \mathrm{EE}=1.71 \pm 0.39 \\
\mathrm{~W}=4.32 \pm 0.96, \text { control }(\mathrm{BHT})=9.63 \pm 0.15\end{array}$ & \\
\hline & St & $\begin{array}{l}\mathrm{Bu}, \mathrm{E}, \mathrm{EE}, \\
\mathrm{Aq}\end{array}$ & Antioxidant- HO (In vitro) & $\begin{array}{c}\mathrm{IC}_{50}(\mu \mathrm{g} / \mathrm{mL}): \mathrm{Bu}=9.07 \pm 0.99, \mathrm{E}=17.93 \pm 1.51, \mathrm{EE}=13.57 \pm 1.59 \\
\mathrm{~W}=33.59 \pm 1.66, \text { control }(\mathrm{BHT})=45.58 \pm 2.14\end{array}$ & \\
\hline & B & CE & \multirow{4}{*}{$\begin{array}{l}\text { Antimicrobial- Disc diffusion } \\
\text { (In vitro) }\end{array}$} & $\begin{array}{c}\text { Activity tested with MT. Complete inhibition with PM, AC, SE, YE, } \\
\text { SA, PA, and BC. Partial inhibition with EC, BS, CA, and CN. No } \\
\text { fungal activity reported }\end{array}$ & \multirow{4}{*}{ [133] } \\
\hline & \multirow{3}{*}{ B } & \multirow{3}{*}{$\mathrm{Me}$} & & $\begin{array}{l}\text { Activity tested with CT. Complete inhibition with SS, SA, PA, and SC. } \\
\text { Partial inhibition with PM, SM, SP, BL, SE, BC, ETA, CA, and CN. No } \\
\text { fungal activity reported }\end{array}$ & \\
\hline & & & & $\begin{array}{l}\text { Activity tested with HT. Complete inhibition with PM, AC, SS, AA, } \\
\text { BL, SE, ST, SA, and CA. Partial inhibition with PA, BC, ETA, RR, and } \\
\text { CN. No fungal activity reported }\end{array}$ & \\
\hline & & & & $\begin{array}{c}\text { MIC (mg/mL): } 1.56 \text { against } \mathrm{AC}, 3.12 \text { against } \mathrm{BC}, 6.25 \text { against } \mathrm{PA}, 6.25 \\
\text { against SA, } 3.13 \text { against } \mathrm{SS}\end{array}$ & \\
\hline
\end{tabular}


Table 7. Cont.

\begin{tabular}{|c|c|c|c|c|c|}
\hline Species & Plant Part(s) & Extract & Study/Assays & Activity & Reference \\
\hline & \multirow{4}{*}{ NI } & \multirow{4}{*}{ NI } & Antioxidant- DPPH (In vitro) & $\begin{array}{l}\text { Most potent radical scavengers: catechol, methoxycatechol, syringol. } \\
\text { Their respective } \mathrm{EC}_{50}(\mathrm{mg} / \mathrm{mL}): 0.1239 \pm 0.0004,0.2001 \pm 0.0005,0.2218 \\
\pm 0.0009 . \mathrm{EC}_{50}(\mathrm{mg} / \mathrm{mL}) \text { Ascorbic acid (control) }=0.2562 \pm 0.0023\end{array}$ & \multirow{4}{*}{ [134] } \\
\hline & & & Antioxidant- FRAP (In vitro) & $\begin{array}{c}\mathrm{AEAC}(\mathrm{mgAA} / \mathrm{g}): \text { syringol }=635 \pm 35, \text { catechol }=2283 \pm 168 \\
\text { methoxycatehol }=1560 \pm 155\end{array}$ & \\
\hline & & & $\begin{array}{l}\text { Antioxidant- } \\
\text { Phosphomolybdenum } \\
\text { (In vitro) }\end{array}$ & $\begin{array}{c}\mathrm{AEAC}(\mathrm{mgAA} / \mathrm{g}): \text { syringol }=1556 \pm 86, \text { catechol }=1861 \pm 95 \\
\text { methoxycatehol }=2396 \pm 194\end{array}$ & \\
\hline & & & Antioxidant- ABTS (In vitro) & $\begin{array}{c}\text { TEAC }(\mathrm{mgTR} / \mathrm{g}): \text { syringol }=956 \pm 40, \text { catechol }=1022 \pm 53 \\
\text { methoxycatechol }=1039 \pm 51\end{array}$ & \\
\hline & $\mathrm{L}$ & NI & $\begin{array}{l}\text { Anti-HIV- MTT assay } \\
\text { (In vitro) }\end{array}$ & $\begin{array}{c}\mathrm{CC}_{50}(\mu \mathrm{g} / \mathrm{mL})=998.21 \pm 81.57, \mathrm{EC}_{50}(\mu \mathrm{g} / \mathrm{mL})=108.55 \pm 16.24 \\
\mathrm{SI}=9.19\end{array}$ & [87] \\
\hline & \multirow{2}{*}{ B } & \multirow{2}{*}{ NI } & Antioxidant- FRAP (In vitro) & $\begin{array}{l}\text { Reducing power increased as concentration of mangrove tannins } \\
\text { increased from } 20 \text { to } 60 \mu \mathrm{g} / \mathrm{mL}\end{array}$ & \multirow{3}{*}[135,136]{} \\
\hline & & & Antioxidant- DPPH (In vitro) & $\begin{array}{l}\text { Scavenging activity increased as concentration of tannins increased. } \\
\text { Maximum scavenging activity }(>90 \%) \text { exhibited at } 30 \mu \mathrm{g} / \mathrm{mL}\end{array}$ & \\
\hline & NI & NI & $\begin{array}{l}\text { Antimicrobial- Disc diffusion } \\
\text { (In vitro) }\end{array}$ & $\begin{array}{l}\text { Zone of inhibition }(\mathrm{mm}) \text { against } \mathrm{BC}=14, \mathrm{SS}=9 . \text { For bacteria, } \mathrm{AC}, \mathrm{KP}, \\
\mathrm{BS}, \mathrm{SA}, \mathrm{BL}, \mathrm{SE}, \mathrm{BC}, \mathrm{SM}, \mathrm{PA}, \mathrm{MIC}(\mathrm{mg} / \mathrm{mL}) \text { ranged from } 3.13 \text { to } 386.25\end{array}$ & \\
\hline \multirow{7}{*}{$\begin{array}{l}\text { Rhizophora mucronata } \\
\text { Lam. }\end{array}$} & \multirow{2}{*}{$\mathrm{L}, \mathrm{Sb}, \mathrm{R}$} & \multirow{2}{*}{$\mathrm{Me}$} & Antioxidant- FRAP (In vitro) & $\begin{array}{c}\text { AAE }(\mathrm{mg} / \mathrm{g}) \text { for the } 3 \text { methanolic extracts of each plant parts }=2.89 \pm \\
0.23,3.62 \pm 0.16 \text {, and } 1.40 \pm 0.00 \text {, respectively }\end{array}$ & \multirow{2}{*}{ [105] } \\
\hline & & & Antioxidant- DPPH (In vitro) & $\begin{array}{c}\mathrm{IC}_{50}(\mathrm{mg} / \mathrm{g}) \text { for the } 3 \text { methanolic extracts of each plant parts }=365.37 \pm \\
23.95,193.82 \pm 11.14, \text { and } 1377.45 \pm 50.62 \text {, respectively }\end{array}$ & \\
\hline & \multirow{5}{*}{$\mathrm{L}$} & \multirow{5}{*}{$\mathrm{C}$} & Antioxidant- DPPH (In vitro) & $\mathrm{IC}_{50}(\mathrm{mg} / \mathrm{mL})=1.38 \pm 0.03$ & \multirow{5}{*}{ [137] } \\
\hline & & & Antioxidant- ABTS (In vitro) & $\mathrm{IC}_{50}(\mathrm{mg} / \mathrm{mL})=1.25 \pm 0.01$ & \\
\hline & & & $\begin{array}{l}\text { Anti-inflammatory- COX-1 } \\
\text { inhibition (In vitro) }\end{array}$ & $\mathrm{IC}_{50}(\mathrm{mg} / \mathrm{mL})=1.42 \pm 0.01$ & \\
\hline & & & $\begin{array}{l}\text { Anti-inflammatory- COX-2 } \\
\text { inhibition (In vitro) }\end{array}$ & $\mathrm{IC}_{50}(\mathrm{mg} / \mathrm{mL})=1.38 \pm 0.00$ & \\
\hline & & & $\begin{array}{l}\text { Anti-inflammatory- 5-LOX } \\
\text { inhibition (In vitro) }\end{array}$ & $\mathrm{IC}_{50}(\mathrm{mg} / \mathrm{mL})=1.16 \pm 0.02$, least active with COX-1 & \\
\hline
\end{tabular}


Table 7. Cont.

\begin{tabular}{|c|c|c|c|c|c|}
\hline Species & Plant Part(s) & Extract & Study/Assays & Activity & Reference \\
\hline \multirow{12}{*}{$\begin{array}{l}\text { Rhizophora mucronata } \\
\text { Lam. }\end{array}$} & \multirow{2}{*}{$\mathrm{L}$} & \multirow{2}{*}{ EA } & \multirow{2}{*}{$\begin{array}{l}\text { Antibacterial- Agar well } \\
\text { diffusion (In vitro) }\end{array}$} & $\begin{array}{l}\text { With } 50 \mu \mathrm{l} \text { of extract, zone of inhibition (mm) against EC, SA, KP, PV, } \\
\text { PA, PSF, ST, and BS }=15,18,9,11,13,9,13 \text {, and } 6 \text {, respectively }\end{array}$ & \multirow{2}{*}{ [138] } \\
\hline & & & & $\begin{array}{c}\text { MIC for EC, SA, KP, PV, PA, PSF, ST, and BS }=8,9,8,15,8,13,11 \text {, and } \\
\qquad 13 \text {, respectively }\end{array}$ & \\
\hline & $\mathrm{R}$ & $\mathrm{H}$ & \multirow{2}{*}{$\begin{array}{l}\text { Antimicrobial- Disc diffusion } \\
\text { (In vitro) }\end{array}$} & $\begin{array}{l}\text { Zone of inhibition (mm) against BS, SA, PA, PV, CA, AFM, and } \\
\text { AN }=20,16,19,17,16,17 \text {, and 18, respectively }\end{array}$ & \multirow{2}{*}{ [99] } \\
\hline & $\mathrm{R}$ & $\mathrm{Me}$ & & $\begin{array}{l}\text { Zone of inhibition (mm) against BS, SA, PA, PV, CA, AFM, and } \\
\qquad \mathrm{AN}=16,14,16,16,14,12 \text {, and 14, respectively }\end{array}$ & \\
\hline & $\mathrm{L}$ & $\mathrm{Me}$ & $\begin{array}{l}\text { Antidiabetic- STZ induced } \\
\text { diabetic rats (In vivo) }\end{array}$ & $\begin{array}{l}\text { Week 3: FBG(mg/100 mL blood) level at } 50 \text { and } 100 \mathrm{mg} / \mathrm{kg}=90.8 \pm \\
6.03 \text { and } 99.3 \pm 4.15 \text {, respectively } \\
\text { Week 10: FBG (mg/100 mL blood) level at } 50 \text { and } 100 \mathrm{mg} / \mathrm{kg}=151 \pm \\
3.26 \text { and } 136 \pm 5.11 \text {, respectively }\end{array}$ & \multirow[t]{2}{*}[64]{} \\
\hline & $\mathrm{L}$ & $\mathrm{Me}$ & Antioxidant - DPPH (In vitro) & $\mathrm{IC}_{50}(\mu \mathrm{g} / \mathrm{mg})=5.25 \pm 0.039$ & \\
\hline & \multirow{3}{*}{$\mathrm{L}$} & \multirow{3}{*}{$\mathrm{Me}$} & $\begin{array}{l}\text { Antibacterial-Disc diffusion } \\
\text { (In vitro) }\end{array}$ & $\begin{array}{c}\text { Zone of inhibition }(\mathrm{mm}) \text { against } \mathrm{BS}, \mathrm{SA}, \mathrm{STF}, \mathrm{STP}, \mathrm{EC} \text {, and PA }=9.97 \\
\pm 0.17,19.56 \pm 0.19,15.74 \pm 0.06,11.31 \pm 0.25,5.63 \pm 0.06 \text {, and } \\
16.57 \pm 0.22 \text {, respectively }\end{array}$ & \multirow{3}{*}{ [139] } \\
\hline & & & Antioxidant- DPPH (In vitro) & $\begin{array}{c}\text { \%radical scavenging at } 4,8,16,32 \text {, and } 64 \mu \mathrm{g} / \mathrm{mL}=15.1 \pm 0.2,19.82 \pm \\
0.61,25.98 \pm 0.46,36.98 \pm 0.04 \text {, and } 42.98 \pm 0.28 \text {, respectively }\end{array}$ & \\
\hline & & & Antioxidant- HO (In vitro) & $\begin{array}{l}\text { \%radical scavenging at } 4,8,16,32 \text {, and } 64 \mu \mathrm{g} / \mathrm{mL}=19.08 \pm 0.14,22.62 \\
\pm 0.35,25.43 \pm 0.18,28.36 \pm 0.22 \text {, and } 32.77 \pm 0.44 \text {, respectively }\end{array}$ & \\
\hline & $\mathrm{L}$ & $\mathrm{C}$ & Analgesic (In vivo) & $\begin{array}{l}\text { Basal reaction time }(\mathrm{s}) \text { after } 15 \mathrm{~min} \text { of administration }=7.40 \pm 0.30 \text {, } \\
\text { after } 30 \mathrm{~min}=11.34 \pm 0.05 \text {, after } 45 \mathrm{~min}=13.13 \pm 0.03 \text {, after } 90 \mathrm{~min}= \\
9.01 \pm 0.28\end{array}$ & [140] \\
\hline & \multirow[b]{2}{*}{ B, F, Fr, L, R } & \multirow[b]{2}{*}{$\mathrm{Me}$} & Antibacterial- Disc diffusion & $\begin{array}{l}\text { Zone of inhibition }(\mathrm{mm}) \text { against SA for the respective plant parts } \\
\text { extracts }=8.8,7.5,7.1,6.1 \text {, and } 7.6\end{array}$ & \multirow[b]{2}{*}[60]{} \\
\hline & & & & $\begin{array}{c}\text { Zone of inhibition }(\mathrm{mm}) \text { against EC for the respective plant parts } \\
\text { extracts }=6.4, \mathrm{NR}, 8.6,6.2 \text {, and } 7.1 . \text { Highest activity with bark extract } \\
\text { for both bacteria }\end{array}$ & \\
\hline
\end{tabular}


Table 7. Cont.

\begin{tabular}{|c|c|c|c|c|c|}
\hline Species & Plant Part(s) & Extract & Study/Assays & Activity & Reference \\
\hline \multirow{18}{*}{$\begin{array}{l}\text { Rhizophora mucronata } \\
\text { Lam. }\end{array}$} & $\mathrm{L}$ & NI & $\begin{array}{c}\text { Anti HIV- MTT assay } \\
\text { (In vitro)Antioxidant- DPPH } \\
\text { (In vitro) }\end{array}$ & $\begin{array}{c}\mathrm{CC}_{50}(\mu \mathrm{g} / \mathrm{mL})=798.39 \pm 72.02, \mathrm{EC}_{50}(\mu \mathrm{g} / \mathrm{mL})=492.29 \pm 48.99 \\
\mathrm{SI}=1.62\end{array}$ & [87] \\
\hline & \multirow{5}{*}{$\mathrm{L}$} & \multirow{5}{*}{$\mathrm{Me}$} & Antioxidant- DPPH (In vitro) & $\mathrm{IC}_{50}(\mu \mathrm{g} / \mathrm{mL})=47.39 \pm 0.43$ & \multirow{5}{*}{ [100] } \\
\hline & & & Antioxidant- HO (In vitro) & $\mathrm{IC}_{50}(\mu \mathrm{g} / \mathrm{mL})=401.45 \pm 18.52$ & \\
\hline & & & Antioxidant- NO (In vitro) & $\mathrm{IC}_{50}(\mu \mathrm{g} / \mathrm{mL})=80.23 \pm 0.70$ & \\
\hline & & & $\begin{array}{l}\text { Antioxidant- Hydrogen } \\
\text { peroxide (In vitro) }\end{array}$ & $\mathrm{IC}_{50}(\mu \mathrm{g} / \mathrm{mL})=316.47 \pm 3.56$ & \\
\hline & & & Anti-cholinesterase (In vitro) & $\begin{array}{l}\text { \%inhibition against } \mathrm{AChE}=92.73 \pm 0.54, \mathrm{BuChE}=98.98 \pm 0.17 \\
\mathrm{IC}_{50}(\mu \mathrm{g} / \mathrm{mL}): \mathrm{AChE}=59.31 \pm 0.35, \mathrm{BuChE}=51.72 \pm 0.35\end{array}$ & \\
\hline & \multirow{5}{*}{$\mathrm{L}$} & \multirow{5}{*}{$\mathrm{Me}$} & Antioxidant- DPPH (In vitro) & $\mathrm{IC}_{50}(\mu \mathrm{g} / \mathrm{mg})=5.25 \pm 0.039$ & \multirow{5}{*}[64]{} \\
\hline & & & Antioxidant- NO (In vitro) & $\mathrm{IC}_{50}(\mu \mathrm{g} / \mathrm{mg})=3.44 \pm 0.038$ & \\
\hline & & & Antioxidant- SO (In vitro) & $\mathrm{IC}_{50}(\mu \mathrm{g} / \mathrm{mg})=6.04 \pm 0.012$ & \\
\hline & & & Antioxidant- HO (In vitro) & $\mathrm{IC}_{50}(\mu \mathrm{g} / \mathrm{mg})=5.01 \pm 0.072$ & \\
\hline & & & Antioxidant- ABTS (In vitro) & $\mathrm{IC}_{50}(\mu \mathrm{g} / \mathrm{mg})=1.42 \pm 0.009$ & \\
\hline & \multirow{2}{*}{ St } & \multirow{2}{*}{$\mathrm{E}$} & \multirow{2}{*}{ Antimicrobial (In vitro) } & $\begin{array}{l}\text { Zone of inhibition (mm) against EC, SA, ST, STP, and PA }=16,15,20 \text {, } \\
12 \text {, and } 15 \text {, respectively. No inhibition against } \mathrm{KP}, \mathrm{PV} \text {, and CA }\end{array}$ & \multirow{2}{*}{ [141] } \\
\hline & & & & 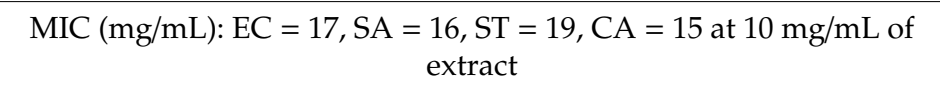 & \\
\hline & $\mathrm{L}$ & \multirow{2}{*}{$\begin{array}{l}\mathrm{H}, \mathrm{EA}, \\
\mathrm{Me}\end{array}$} & \multirow{2}{*}{ Anti-cholinesterase (In vitro) } & $\begin{array}{c}\mathrm{IC}_{50}(\mu \mathrm{g} / \mathrm{mL}): \mathrm{H}=\mathrm{NR}, \mathrm{EA}=\mathrm{NR}, \mathrm{Me}=222.48, \text { Physostigmine } \\
(\text { (control) }=0.06\end{array}$ & \multirow{2}{*}[142]{} \\
\hline & $\mathrm{Fr}$ & & & $\begin{array}{c}\mathrm{IC}_{50}(\mu \mathrm{g} / \mathrm{mL}): \mathrm{H}=3.68 \times 10^{-6}, \mathrm{EA}=322.27, \mathrm{Me}=1.01, \text { Physostigmine } \\
\text { (control) }=0.06\end{array}$ & \\
\hline & $\mathrm{AP}$ & EA & \multirow{3}{*}{$\begin{array}{l}\text { Antimicrobial- Agar disc } \\
\text { diffusion (In vitro) }\end{array}$} & Overall activity (\%) against SA, SM, KP, SF, ML, VM = 66.6 & \multirow{3}{*}{ [143] } \\
\hline & $\mathrm{AP}$ & $\mathrm{Me}$ & & Overall activity (\%) against SA, SM, KP, SF, ML, VM = 100.0 & \\
\hline & $\mathrm{AP}$ & $\mathrm{C}$ & & Overall activity (\%) against $\mathrm{SA}, \mathrm{SM}, \mathrm{KP}, \mathrm{SF}, \mathrm{ML}, \mathrm{VM}=14.28$ & \\
\hline
\end{tabular}


Table 7. Cont

\begin{tabular}{|c|c|c|c|c|c|}
\hline Species & Plant Part(s) & Extract & Study/Assays & Activity & Reference \\
\hline \multirow{3}{*}{$\begin{array}{l}\text { Rhizophora mucronata } \\
\text { Lam. }\end{array}$} & Fr & NI & $\begin{array}{c}\text { Antidiabetic- Alloxan- } \\
\text { induced diabetic rats (In vivo) }\end{array}$ & $\begin{array}{c}\text { Dosage 500, 1000, } 1500,2000 \mathrm{mg} / \text { day/head for } 18 \text { days were } \\
\text { administered into diabetic rats } \\
\text { Positive control (glibenclamide: } 0.09 \mathrm{mg} / \text { day } / 200 \mathrm{~g} \text { body weight) } \\
\text { Blood glucose level of both groups (control and experimental rat } \\
\text { group) decreased }\end{array}$ & [144] \\
\hline & $\mathrm{L}$ & NI & $\begin{array}{l}\text { Antidiabetic- Alloxan- } \\
\text { induced diabetic rats (In vivo) }\end{array}$ & $\begin{array}{c}\text { Dosage } 60 \mathrm{mg} / \mathrm{kg} \text { was administered to rats for } 30 \text { days. A decrease in } \\
\text { blood glucose level was observed }\end{array}$ & [145] \\
\hline & $\mathrm{L}$ & $\mathrm{E}$ & $\begin{array}{l}\text { Hypoglycemic effect- } \\
\text { Streptozotocin-induced } \\
\text { diabetic rats (In vivo) }\end{array}$ & $\begin{array}{l}\text { Dosage } 100 \text { and } 200 \mathrm{mg} / \mathrm{kg} \text { were administered for } 6 \mathrm{~h} \text {. } \\
\text { Positive control (glibenclamide) }=0.5 \mathrm{mg} / \mathrm{kg} \text {. Higher percentage } \\
\text { decrease observed with control }(27.2 \%) \text { compared to } 100 \mathrm{mg} / \mathrm{kg} \\
\text { extract }(19.7 \%), 200 \mathrm{mg} / \mathrm{kg} \text { extract }(21.0 \%)\end{array}$ & [146] \\
\hline $\begin{array}{l}\text { Rhizophora racemosa G. } \\
\text { Mey }\end{array}$ & $\mathrm{L}$ & $\mathrm{Me}$ & $\begin{array}{l}\text { Lethal dose evaluation- } \\
\text { Karber's method (In vitro) }\end{array}$ & $\begin{array}{c}\mathrm{LD}_{50}=1583.33 \mathrm{mg} / \mathrm{kg} \text {, the lethal dose is safe to use as a traditional } \\
\text { medicine }\end{array}$ & [102] \\
\hline \multirow{2}{*}{ Rhizophora stylosa Griff. } & $\mathrm{L}$ & $\begin{array}{l}\text { H, EA, } \\
\mathrm{Me}\end{array}$ & \multirow{2}{*}{ Anti-cholinesterase (In vitro) } & $\begin{array}{c}\mathrm{IC}_{50}(\mu \mathrm{g} / \mathrm{mL}): \mathrm{H}=715.52, \mathrm{EA}=\mathrm{NR}, \mathrm{Me}=268.39, \text { Physostigmine } \\
\text { (control) }=0.06\end{array}$ & \multirow{2}{*}{ [142] } \\
\hline & $\mathrm{Fr}$ & $\begin{array}{l}\mathrm{H}, \mathrm{EA} \\
\mathrm{Me}\end{array}$ & & $\begin{array}{c}\mathrm{IC}_{50}(\mu \mathrm{g} / \mathrm{mL}): \mathrm{H}=\mathrm{NR}, \mathrm{EA}=2.92, \mathrm{Me}=9.56, \text { Physostigmine (control) } \\
=0.06\end{array}$ & \\
\hline \multirow{6}{*}{$\begin{array}{c}\text { Xylocarpus granatum J. } \\
\text { Koenig }\end{array}$} & \multirow{3}{*}{ NI } & \multirow{3}{*}{ NI } & Antioxidant- DPPH (In vitro) & $\mathrm{IC}_{50}(\mu \mathrm{M})=3.3 \pm 0.3$ & \multirow{5}{*}[147]{} \\
\hline & & & Antioxidant- 15LOX (In vitro) & $\mathrm{IC}_{50}(\mu \mathrm{M})=9 \pm 1$ & \\
\hline & & & Anticancer (In vitro) & $\mathrm{IC}_{50}(\mu \mathrm{M})$ of 16.93 against $\mathrm{CaCo}-2$ colon cancer cell line & \\
\hline & $\mathrm{B}$ & $\mathrm{Me}$ & Antidiarrheal (In vivo) & $\begin{array}{c}\text { Significant activity at doses } 250 \text { and } 500 \mathrm{mg} / \mathrm{kg} \text { against castor oil and } \\
\text { magnesium sulfate induced murine models }\end{array}$ & \\
\hline & $\mathrm{B}, \mathrm{L}, \mathrm{Fr}$ & $\mathrm{E}$ & $\begin{array}{l}\text { Antidiarrheal- Castor oil } \\
\text { induced diarrheal model } \\
\text { (In vivo) }\end{array}$ & Active & \\
\hline & $\mathrm{Sb}$ & $\mathrm{E}$ & $\begin{array}{l}\text { Antimicrobial- Agar disc } \\
\text { diffusion (In vitro) }\end{array}$ & Active against EC, ETA, PA, ST, SA VC, and KP & {$[104]$} \\
\hline
\end{tabular}


$\mathrm{CN}=$ Cryptococcus neoformans, $\mathrm{COX}=$ Cyclooxigenase, $\mathrm{CT}=$ Condensed tannin, $\mathrm{CS}=$ Citrobacter sp, $\mathrm{DPPH}=1$-diphenyl-2-picryhydrazyl $, \mathrm{E}=\mathrm{Ethanol}, \mathrm{EA}=\mathrm{Ethyl}$ acetateextract, $\mathrm{EE}$

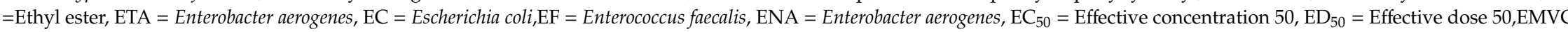
= Encephalmyocarditis virus, ETA = Enterobacter aerogenes, $\mathrm{FBG}=$ Fast blood glucose, $\mathrm{Fr}=$ Fruit, FRAP $=$ Ferric reducing antioxidant power, GaIN $=\mathrm{D}$-galactosamine, $\mathrm{H}=\mathrm{Hexane}$ extract, $\mathrm{HBV}=$ Hepatitis B virus, HIV = Human immunodeficiency virus, HL-60 = Human leukaemic 60, HO = Hydroxyl, HT = Hydrolysable tannin, Hy = Hypocotyl, IC $50=$ Inhibitory concentration 50, KP = Klebsiella pneumonia, $\mathrm{KS}=$ Klebsiella sp, L = Leaf, LA = Lactobacillus acidophilus, LD = Lactobacillus delbrueckii, LDV = Leishmania donovani, LOX = lipoxygenase, MDA = Malondialdehyde, $\mathrm{Me}=$ Methanol, MIC = Minimun inhibitory concentration, ML = Micrococcus luteus, MTT = 3-(4, 5-dimethylthiazol-2-yl)-2, 5-diphenyltetrazolium bromide, MT = Mixed tannin, MTS $=$ Cell proliferation assay, $\mathrm{NI}=$ Not indicated, $\mathrm{NO}=$ Nitric oxide, $\mathrm{NR}=$ No result, $\mathrm{PA}=$ Pseudomonas aeruginosa, $\mathrm{PE}=\mathrm{Petroleum}$ ether extract, $\mathrm{PF}=$ Plasmodium falciparum, $\mathrm{PM}=$ Proteus mirabilis, $\mathrm{PT}=$ Prothrombin time, $\mathrm{PSF}=$ Pseudomanas fluorescens, $\mathrm{PV}=$ Proteus vulgaris, $\mathrm{PS}=$ Proteus $\mathrm{sp}, \mathrm{REMA}=\mathrm{Resazurin}$ microtitreassay, $\mathrm{R}=\mathrm{Root}, \mathrm{RPA}=\mathrm{Raw}$ pyroligeneous acid, $\mathrm{RR}=$ Rhodotorula rubra, $\mathrm{S}=\mathrm{Seed}, \mathrm{SA}=$ Staphylococcus aureus, $\mathrm{Sb}=\mathrm{Stem}$ bark, $\mathrm{SE}=$ Staphylococcus epidermidis, $\mathrm{SF}=\mathrm{Shigella}$ flexneri, $\mathrm{SFV}=\mathrm{Semliki}$ forest virus, $\mathrm{SI}=$ Selective index $\left(\mathrm{CC}_{50} / \mathrm{EC}_{50}\right), \mathrm{SC}=$ Staphylococcus cerevisiae, $\mathrm{SD}=$ Shigella dysenteriae, $\mathrm{SM}=$ Serratia marcesens, $\mathrm{SP}=$ Salmonella paratyphi, $\mathrm{SS}=$ Staphylococcus saprophyticus, $\mathrm{ST}=$ Salmonella typhi $\mathrm{STF}=$ Streptococcus faecalis, $\mathrm{STM}=$ Streptococcus mutans, $\mathrm{STP}=$ Streptococcus pyogenes, $\mathrm{STZ}=$ Streptozotocin, $\mathrm{STS}=$ Streptococcus salivarius, $\mathrm{St}=\mathrm{Stem}, \mathrm{SO}=\mathrm{Superoxide}, \mathrm{TC}=$ Trypanosoma cruzi, $\mathrm{TEAC}=\mathrm{mg}$ of Trolox equivalents per gram sample, $\mathrm{TR}=$ Tricophyton rubrum, $\mathrm{VC}=$ Vibrio cholera, $\mathrm{VC}=$ Vibrio mimicus, $\mathrm{YE}=$ Yersinia enterocolitica. 
Figure 8 illustrates the types of assays usually conducted on mangroves. It is evident that antioxidant $(28.8 \%)$ and antimicrobial $(24.0 \%)$ assays were the two most common in vitro studies performed. Interestingly, most in vivo studies were done for antidiabetic assays compared to in vitro. It is found that antipyretic, antiviral, thrombolytic activity, anticoagulant, antiparasitic, antiulcer, and anti-filarial tests were less seldom conducted. However, it is important to highlight that many mangrove species are used as a remedy for the ulcer in folklore medicine. For instance, the leaf of A. marina, the leaf of A. officinalis, the bark of B. cylindrica, bark, fruit, and leaf of $C$. decandra, whole plant of C. roxburghiana, and whole plant of R. mucronata (Table 5) are traditionally believed to cure ulcers. Nonetheless, the antiulcer potential of these named plants has not been extensively validated either in vivo or in vitro studies to confirm this belief in medical lore.

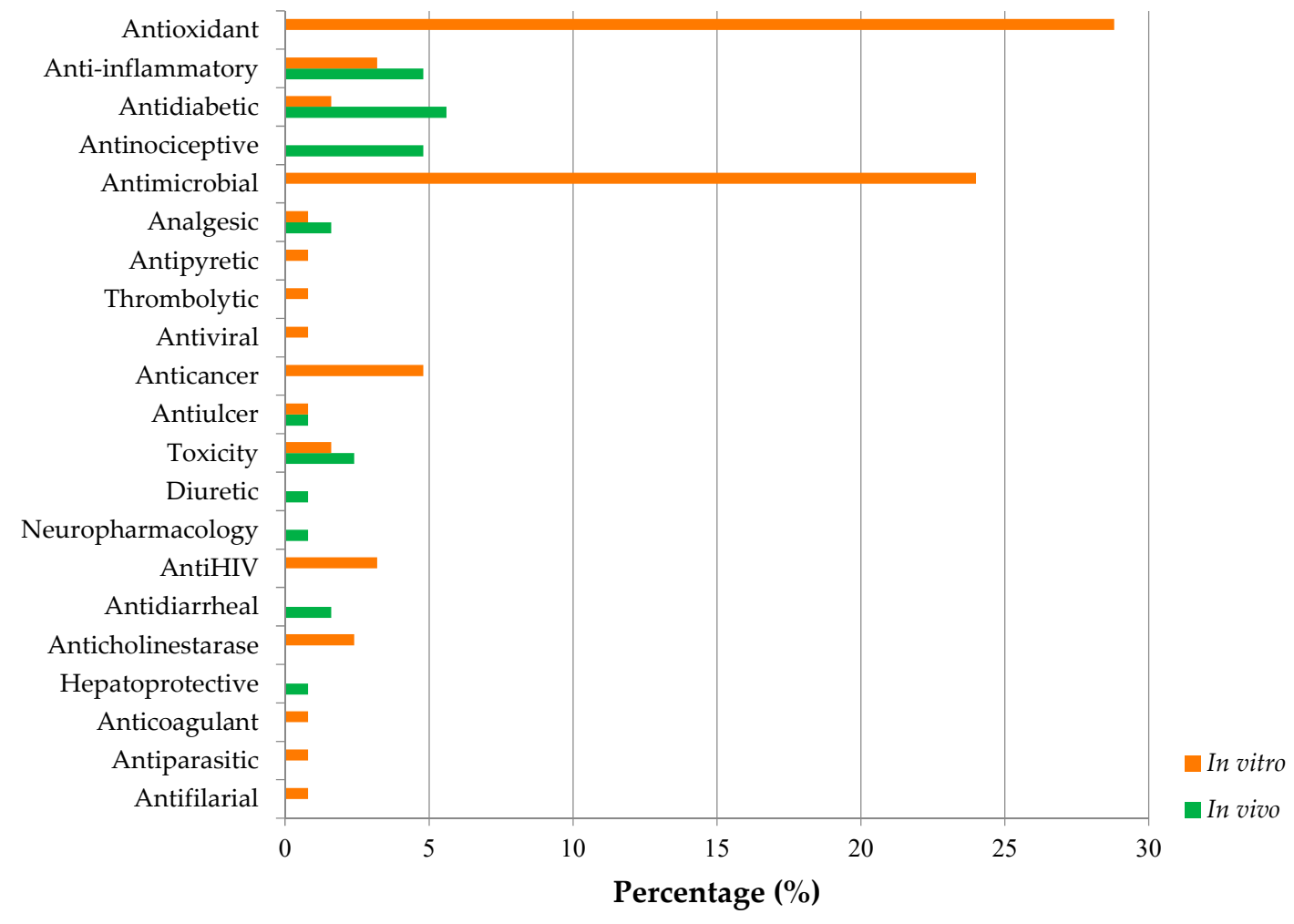

Figure 8. Types of assays.

A pie chart in Figure 9 represents mangroves that have been pharmacologically validated. The five most reportedly investigated species are R. mucronata (19\%), A. officinalis $(11 \%)$, A. marina (9\%), B. gymnorhiza (8\%), and R. apiculata (7\%). It is important to highlight that $B$. gymnorhiza is the most traditionally used species (Figure 9), but it is found in the fourth place to be pharmacologically validated. This warrants an in-depth study on that particular species since its importance in folklore medicine. 

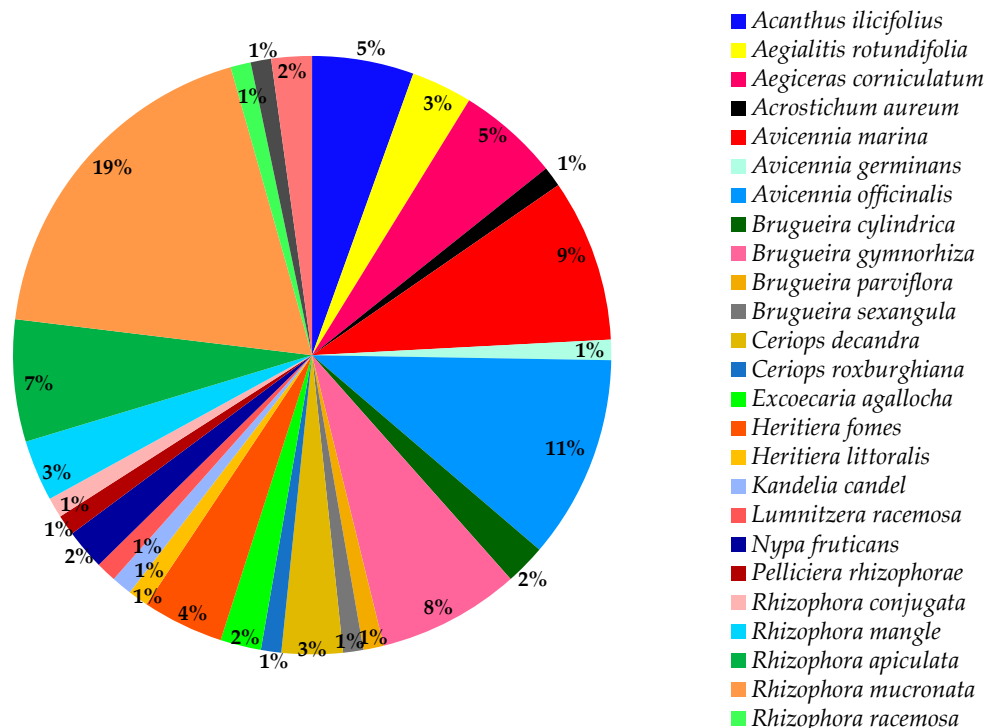

Figure 9. Pharmacologically validated species of mangroves.

\section{Phytochemistry of Mangroves}

Plants possess a plethora of novel and biologically active secondary metabolites and thus serves a reservoir for the production of novel drug compounds. In this era in which most researchers are screening thousands of plants for the discovery of novel compounds, it is thus of high importance to scrutinize mangrove species with that very aim to isolate new phytochemicals which can be potential candidates for the development of pharmaceutical drugs. Saying so, about 200 bioactive metabolites have already been identified from mangroves $[36,148]$. Therefore, this prompts more studies for phytochemical screening of new metabolites. Phytochemical studies conducted on various mangrove species are summarized in Table 8. Histogram in Figure 10 illustrates the 16 most common types of phytochemicals isolated from mangrove species. Generally, the seven most common chemical constituents present are terpenoids $(16.25 \%)$, tannins $(12.5 \%)$, steroids $(10.0 \%)$, alkaloids $(9.38 \%)$, flavonoids $(8.75 \%)$, saponins $(8.75 \%)$, and glycosides $(8.13 \%)$. Furthermore, mangroves also yielded other compounds namely fatty acid derivative, anthraquinone, amino acid, coumarin, quinine, ester, gum, phenol, terpene quercetin, and anthranoid. However, these compounds are found at low levels and are present in only certain mangrove plants. For example, the presence of fatty acids has been reported only in A. ilicifolius and A. marina but not in any other species (Table 8).

There is an undeviating link between phytochemicals and pharmacological activities. Kathiresan et al. [149] have shown that the bioactive compounds such as galactose, galactosamine, glucose, and arabinose possess significant anti-HIV activity. The different types of constituents present in medicinal plants are responsible for the wide range of pharmacological activities that the plants possess. It is reported that plants grown along the coastal regions are known to be potential resources of anticancer drugs [149]. For instance, the constituent tannin isolated from the species B. sexangula showed anticancer activity against Lewis lung carcinoma and Sarcoma 180 [149,150]. Additionally, the bark extracts of this mangrove species have shown antitumor activity which was due to the tannin-free aqueous residue containing the alkaloid, brugine, tropine, and its acetic ester acid [149]. Compounds produced by the species R. mangle also showed potent activity against carcinomas, melanomas, and lymphomas [149]. 


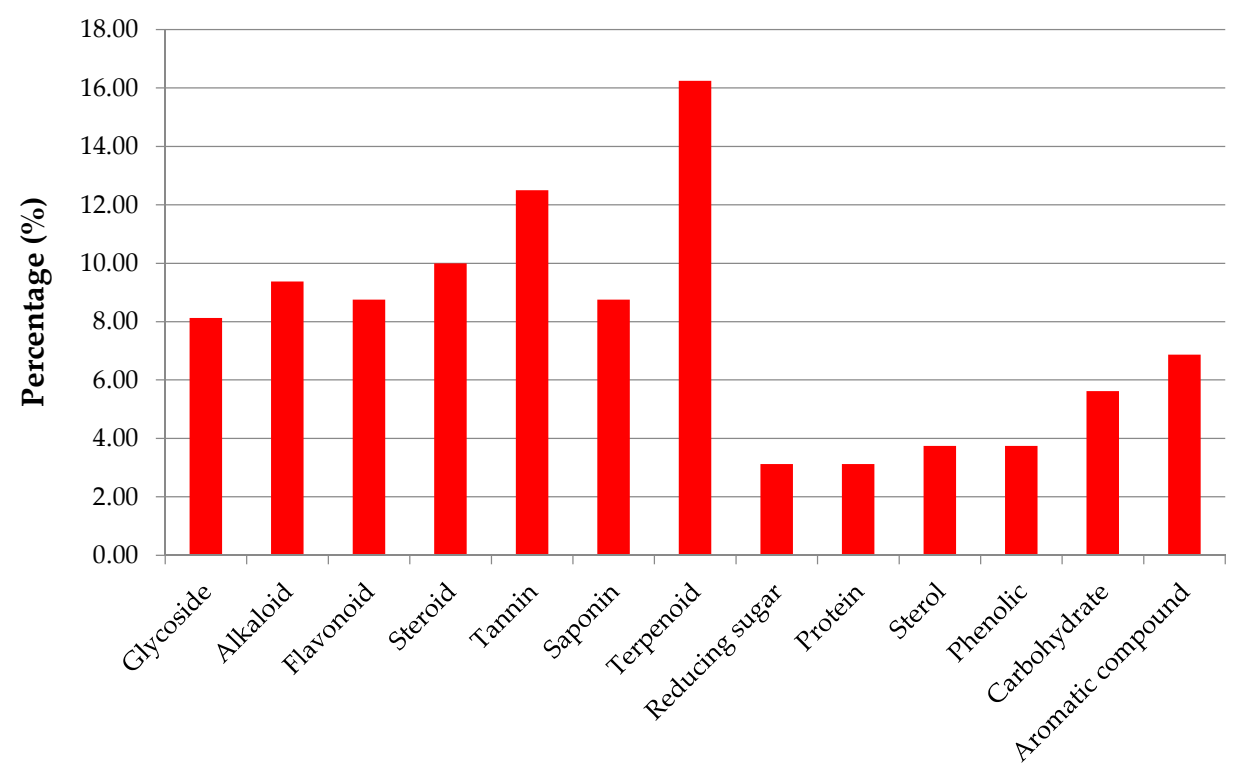

Figure 10. Classes of compounds isolated from mangroves.

Barik et al. [121] were the first to isolate a flavone known as 5,7-dihydroxy-2-(3-hydroxy-4, 5-dimethoxy-phenyl)-chromen-4-one-a, from the leaves of B. gymnorhiza. It has been reported that the compound was responsible for anti-inflammatory activity with a percentage inhibition of $80 \%$ against COX-2 mediated prostaglandin E2 production. Phenol group is a bioactive chemical compound that shows good antioxidant activity. High antioxidant activity was exhibited by the methanolic fruit extract of B. gymnorhiza with an $\mathrm{IC}_{50}$ value of $13.47 \mathrm{ppm}$. The fruit is rich in carbohydrate $(29.28 \%)$ and thus can become a potential food source [64,151]. Sur et al. [64] reported that polyphenols such as gallic acid, quercetin and coumarin isolated from the methanolic leaf extract showed significant antioxidant activities. These constituents help in nursing the injury of hepatic tissue through its antioxidant effects. Moreover, constituents mainly flavonoids, reducing sugars, gums, saponins, and tannins isolated from the roots of B. gymnorhiza are responsible for antinociceptive and antidiarrhea properties [152].

Rhizophora mucronata, another popular mangrove species consists of a broad spectrum of chemical constituents such as sugar, tannins, saponins, alkaloids, flavonoids, steroids, terpenoids, glycosides, phenolics $[60,88,153]$, gibberellins, lipids, inositols, anthocyanidins, polysaccharides, proteins, minerals, hydrolysable tannins, and polyphenols (Balasubramanian et al. 2015). From the phytochemical screening test of R. mucronata the alkaloid, rhizophorine is considered as a major component in the leaf of the plant [65]. The methanolic leaf extract exhibited a strong anti-cholinesterase activity (AChE assay) with an $\mathrm{IC}_{50}$ value of $59.31 \pm 0.35 \mu \mathrm{g} / \mathrm{mL}$ and potent antioxidant activity (DPPH) with an $\mathrm{IC}_{50}$ value of $47.39 \pm 0.43 \mu \mathrm{g} / \mathrm{mL}$. These significant results could be attributed to the presence of a high number of flavonoids, particularly catechin (128) [100]. With regards to antidiabetic activity, R. mucronata is considered as an excellent natural antidiabetic agent due to the presence of phenolics, flavonoids, gallic acid (130), quercetin (12), and coumarin [64]. Furthermore, a study by Rohini and Das [154] revealed the excellent anti-inflammatory activity of the bark extract of $R$. mucronata with the presence of the phytoconstituents lupeol (48), quercetin (12), $\beta$-sitosterol (54), and caffeic acid. Manilal et al. [143] are of the view that the main constituent, ethanone (1-(2-hydroxy-5-methylphenyl), isolated from the crude extract could play a pivotal role in the antibiotic activity of the plant. The chemical structures of isolated compounds from mangroves are illustrated in Figures 11-24. 
Table 8. Phytochemical constituents of different mangrove species.

\begin{tabular}{|c|c|c|c|c|c|}
\hline Species & Plant Part & Extract & Phytochemical Class & Constituent & Reference \\
\hline \multirow{10}{*}{ Acanthus ilicifolius $\mathrm{L}$. } & \multirow{10}{*}{ NI } & \multirow{10}{*}{ NI } & Aliphatic glycosides & Ilicifolioside B (1), ilicifolioside C (2) & \multirow{10}{*}{ [42] } \\
\hline & & & Alkaloids & $\begin{array}{l}\text { Acanthicifoline (3), trigonelline (4), 2-benzoxazolinone (5), benzoxazin-3-one (6), } \\
\text { 5,5'-bis-benzoxazoline-2,2'-dione (7), 4-O- } \beta \text {-D-glucopyranosyl-benzoxazolin-2(3H)-one } \\
\text { (8), }(2 R)-2-\beta \text {-D-glucopyranosyloxy-2H-1,4-benzoxazine-3(4H)-one }(9), \\
(2 R)-2-\beta \text {-glucopyranosyloxy-4-hydroxy-1,4-benzoxazine-3-one (10), } \\
\text { 2-hydroxy-2H-1,4-benzoxazin } 3(4 \mathrm{H}) \text { one (11) }\end{array}$ & \\
\hline & & & Flavonoids & $\begin{array}{l}\text { Quercetin (12), quercetin 3-O- } \beta \text {-D-glucopyranoside (13), apigenin 7-O- } \beta \text {-D-glucuronide } \\
\text { (14), methylapigenin 7-O- } \beta \text {-D-glucopyranuronate (15), acacetin } \\
\left.\text { 7-O- } \beta \text {-L-rhamnopyranosyl-(1" } \rightarrow 6^{\prime \prime}\right)-O-\beta \text {-D-glucopyranoside (16), vitexin (17) }\end{array}$ & \\
\hline & & & Lignan glycosides & $\begin{array}{c}\text { (+)-lyoniresinol 3a-(2-(3,5-dimethoxy-4-hydroxy)-benzoyl)-O- } \beta \text {-glucopyranoside (18), } \\
\text { dihydroxymethyl-bis(3,5-dimethoxy-4-hydroxyphenyl)tetrahydrofuran-9(or } \\
\text { 9')-O- } \beta \text {-D-glucopyranosid (19), } \\
\left(8 R, 7^{\prime} S, 8^{\prime} R\right)-5,5^{\prime} \text {-dimethoxylariciresinol-4-O- } \beta \text {-D-glucopyranoside (20), alangilignoside C } \\
(21),(+) \text {-syringaresinol-O- } \beta \text {-D-glucopyranoside }(22),(+) \text { lyoniresinol } \\
\text { 3-O- } \beta \text {-D-glucopyranoside (23), (+)-lyoniresinol } \\
\text { 2a-O- } \beta \text {-D-galactopyranosyl-3a-O- } \beta \text {-D-glucopyranoside (24), (+)-lyoniresinol } \\
\text { 3a-O- } \beta \text {-D-galactopyranosyl-(1-6)- } \beta \text {-D-glucopyranoside (25), (-)-lyoniresinol } \\
\text { 3-O- } \beta \text {-D-glucopyranoside (26) }\end{array}$ & \\
\hline & & & Megastigmane and phenolic glycosides & $\begin{array}{c}\text { (Z)-4-coumaric acid 4-O- } \beta \text {-D-glucopyranoside }(27),(Z)-4 \text {-coumaric acid } \\
\text { 4-O- } \beta \text {-D-apiofuranosyl-( }\left(1^{\prime \prime} / 2^{\prime}\right) \text {-O-D-glucopyranoside (28), } \\
\text { (6R,7E,9R)-9-hydroxy-megastigman-4,7-dien-3-one-9-O- } \beta \text {-D-glucopyranoside (29), } \\
\text { (6S,7E,9S)-6,9-dihydroxymegastigman-4,7-dien-3-one-9-O- } \beta \text {-D-glucopyranoside (30), } \\
\text { plucheoside B (31), 2,6-dimethoxy-p-hydroquinone 1-O- } \beta \text {-D-glucopyranoside (32), } \\
\text { syringic acid-O- } \beta \text {-D-glucopyranosyl ester (33), 5,11-epoxymegastigmane glucoside (34) }\end{array}$ & \\
\hline & & & Phenylethanol glycosides & $\begin{array}{c}\text { Phenylethyl-O- } \beta \text {-D-glucopyranosyl-(1/2)-O- } \beta \text {-D-glucopyranoside (35), } \\
\text { phenylethyl-O- } \beta \text {-D-glucopyranoside (36), cistanoside F (37), isocistanoside F (38), } \\
\text { cistanoside E (39), campneoside I (40), ilicifolioside A (41), ilicifolioside D (42), acteoside } \\
\text { (43), isoacteoside (44) }\end{array}$ & \\
\hline & & & Triterpenoids & $\begin{array}{l}\alpha \text {-L-Arabinofuranosyl-(1/4)- } \beta \text {-D-glucuronopyranosyl-(1_3)-3-hydroxylup-20(29)-ene } \\
(45), \alpha \text {-amyrin (46), } \beta \text {-amyrin (47), lupeol (48), oleanolic acid (49), ursolic acid (50) }\end{array}$ & \\
\hline & & & Steroids & $\begin{array}{l}\text { Cholesterol (51), campesterol (52), stigmasterol (53), } \beta \text {-sitosterol (54), stigmast-7-en-3-ol } \\
\text { (55), 28-isofucosterol (56), octacosyl alcohol (57), sitosterol-3-O- } \beta \text {-D-glucopyranoside ( } 58 \text { ) }\end{array}$ & \\
\hline & & & Fatty acid derivatives & $\begin{array}{l}\text { Palmitic acid (59), octadecanoic acid (60), stigmasterol octadecenoate (61), } \beta \text { - tetracosanol } \\
\text { (62), octacosanol (63) }\end{array}$ & \\
\hline & & & Miscellaneous & $\begin{array}{l}\text { (2R)-2-O- } \beta \text {-D-glucopyranosyl-4-hydroxy-2H-1,4-benzoxazin-3(4H)-one (64), betaine (65), } \\
\text { vanillic acid (66), luteolin-7-O- } \beta \text {-D-glucuronide (67), uridine (68), uracil (69) }\end{array}$ & \\
\hline
\end{tabular}


Table 8. Cont

\begin{tabular}{|c|c|c|c|c|c|}
\hline Species & Plant Part & Extract & Phytochemical Class & Constituent & Reference \\
\hline \multirow{12}{*}{ Acanthus ilicifolius L. } & NI & $\mathrm{H}, \mathrm{Me}$ & $\begin{array}{l}\text { Anthraquinone, alkaloids, flavonoids, } \\
\text { glycosides, saponins, tannins, } \\
\text { terpenoids }\end{array}$ & NI & [155] \\
\hline & $\mathrm{B}, \mathrm{Fr}, \mathrm{L}, \mathrm{R}$ & NI & $\begin{array}{l}\text { Alkaloids, long chair alcohols, steroids, } \\
\text { sulphur, triterpenes, saponins }\end{array}$ & NI & [88] \\
\hline & $\mathrm{L}$ & $\mathrm{Me}$ & $\begin{array}{l}\text { Protein, resin, steroids, tannins, } \\
\text { glycosides, reducing sugar, } \\
\text { carbohydrates, saponins, sterols, } \\
\text { terpenoids, acidic compounds, phenol, } \\
\text { cardio glycosides, catechol }\end{array}$ & NI & [156] \\
\hline & $\mathrm{L}$ & $\mathrm{Me}$ & $\begin{array}{l}\text { Flavonoids, tannins, steroids, saponins, } \\
\text { glycosides }\end{array}$ & NI & [68] \\
\hline & $\mathrm{R}$ & E & NI & Erigeside C (70) & [157] \\
\hline & $\mathrm{R}$ & E & Triterpenoid saponin & NI & \multirow{6}{*}{ [158] } \\
\hline & $\mathrm{L}$ & $\mathrm{Aq}$ & 2-benzoxazolinone & $\mathrm{NI}$ & \\
\hline & $\mathrm{L}$ & $\mathrm{C}$ & Pentacyclic triterpenoids, sterols & $\mathrm{NI}$ & \\
\hline & $\mathrm{L}$ & $\mathrm{E}$ & $\begin{array}{c}\text { Methylapigenin } \\
\text { 7-O- } \beta \text {-glucoronate-flavone glycosides }\end{array}$ & NI & \\
\hline & $\mathrm{L}$ & $\mathrm{Me}$ & Bisoxazolinone & NI & \\
\hline & AP & $\mathrm{Me}$ & Lignan, cyclolignan glycosides & $\mathrm{NI}$ & \\
\hline & $\mathrm{L}$ & E & NI & 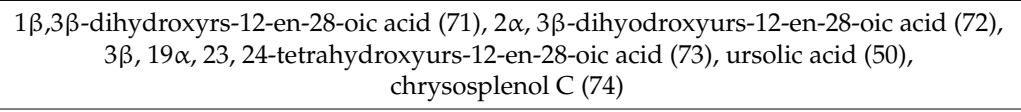 & [159] \\
\hline $\begin{array}{l}\text { Aegialitis rotundifolia } \\
\text { Roxb. }\end{array}$ & $\mathrm{L}$ & E & $\begin{array}{l}\text { Alkaloids, carbohydrates, tannins and } \\
\text { phenolic compounds, steroids, sterols, } \\
\text { triterpenoids, saponins, flavonoids }\end{array}$ & e & [160] \\
\hline \multirow[t]{2}{*}{$\begin{array}{l}\text { Aegiceras corniculatum } \\
\text { (L.) Blanco }\end{array}$} & B, L, St & NI & $\begin{array}{c}\text { Amino acids, benzoquinones, tannins, } \\
\text { coumarins, flavonoids, saponins, } \\
\text { polyphenols, triterpenoids, steroids, } \\
\text { quinines }\end{array}$ & NI & [88] \\
\hline & $\mathrm{L}$ & $\mathrm{Me}$ & $\begin{array}{l}\text { Tannins, saponins, glycosides, } \\
\text { phenolics, flavonoids }\end{array}$ & NI & [108] \\
\hline \multirow[t]{2}{*}{ Acrostichum aureum L. } & $\mathrm{L}$ & $\mathrm{Pe}$ & $\begin{array}{l}\text { Flavonoids, phenols, sterols, phenol, } \\
\text { and polyphenol }\end{array}$ & NI & \multirow[t]{2}{*}{ [76] } \\
\hline & $\mathrm{L}$ & $\mathrm{Me}, \mathrm{W}$ & Flavonoids, phenols & NI & \\
\hline
\end{tabular}


Table 8. Cont.

\begin{tabular}{|c|c|c|c|c|c|}
\hline Species & Plant Part & Extract & Phytochemical Class & Constituent & Reference \\
\hline \multirow{11}{*}{$\begin{array}{l}\text { Avicennia marina } \\
\text { (Forssk.) Vierh. }\end{array}$} & St & NI & $\begin{array}{l}\text { Phytoalexins, tannins, triterpenes, } \\
\text { steroids }\end{array}$ & NI & [88] \\
\hline & $\mathrm{L}$ & $\begin{array}{l}\text { M, E, EE, } \\
\text { EA, W }\end{array}$ & $\begin{array}{l}\text { Alkaloids, glycosides, phenols, } \\
\text { steroids, tannins, terpenoids }\end{array}$ & NI & \multirow{9}{*}{ [7] } \\
\hline & $\mathrm{L}$ & M, EA & Saponins & $\mathrm{NI}$ & \\
\hline & $\mathrm{L}$ & $\begin{array}{l}\text { M, E, EE, } \\
\text { EA, W }\end{array}$ & Flavonoids & $\begin{array}{c}\text { Luteolin 7-O-methylether (75), chrysoeriol 7-oglucoside (76), isorhamnetin 3-O-rutinoside } \\
\text { (77), 5-hydroxy-4; 7-dimethoxyflavone (78), quercetin (12), laempferol (79), } \\
4^{\prime} 5 \text {-dihydroxy-3'-5,7-diimethoxyflavone (80), } 4^{\prime}, 5 \text {-dihydroxy-3',7-trimethoxyflavone (81), } \\
4^{\prime}, 5,7 \text {-trihydroxyflavone }(82), 3^{\prime}, 4^{\prime}, 5 \text {-trihydroxy-7-methoxyflavone (83), } \\
\text { 2-(3'-3'-hydroxymethyloxiran-2'-yl-2' methoxy-4'-Methoxymethylphenyl)-4H } \\
\text { chromen-4-one (84) }\end{array}$ & \\
\hline & $\mathrm{L}$ & $\begin{array}{l}\text { M, E, EE, } \\
\text { EA, W }\end{array}$ & Naphthalene Derivatives & $\begin{array}{c}\text { Naphtha[1,2-b]furan-4,5-dione (85), 3-hydroxy-naphtha[1C-b]furan- 4,5-dione (86), } \\
\text { 2-[2'-2'-hydroxypropyl]-naphtha[1,2-b]furan-4,5-dione (87), avicennone A (88), avicenol } \\
\text { A (89), stenocarpoquinone B }(90), 7^{\prime} S, 8^{\prime} R-4,4^{\prime}, 9^{\prime} \text {-trihydroxy-3,3',5,5'- } \\
\text { tetramethoxy-7,8-dehydro-9-al-2, } 7^{\prime} \text {-cycloligan (91), lyoniresinol (92) }\end{array}$ & \\
\hline & $\mathrm{L}$ & $\begin{array}{l}\text { M, E, EE, } \\
\text { EA, W }\end{array}$ & Tannins & Lapachol (93) & \\
\hline & $\mathrm{L}$ & $\begin{array}{l}\text { M, E, EE, } \\
\text { EA, W }\end{array}$ & Steroids & $\begin{array}{l}\beta \text {-sitosterol (54), ergost-6,22-diene-5,8-epidioxy-3 } 3 \text {-ol (94), } \\
\text { stigmasterol-3-O- } \beta \text {-D-galactopyranoside (95) }\end{array}$ & \\
\hline & $\mathrm{B}, \mathrm{L}, \mathrm{R}$ & $\begin{array}{l}\text { M, E, EE, } \\
\text { EA, W }\end{array}$ & Terpenoids & $\begin{array}{l}\text { Lupeol (48), taraxerol (96), taraxerone (97), betulinic acid (98), betulin (99), ursolic acid } \\
\text { (50), } 6 \mathrm{H} \alpha-11,12,16 \text {-trihydroxy-6,7-secoabieta-8,11,13-triene-6,7-dial11,6-hemiacetal (100), } \\
6 \mathrm{H} \beta-11,12,16 \text {-trihydroxy-6,7-secoabieta-8,11,13-triene-6,7-dial11,6-hemiacetal (101) }\end{array}$ & \\
\hline & $\mathrm{L}, \mathrm{R}$ & $\begin{array}{l}\text { M, E, EE, } \\
\text { EA, W }\end{array}$ & Fatty Acids & $\begin{array}{l}\text { Oleic acid (102), linolenic acid (103), palmitic acid (59), stearic acid (104), lauric acid (105), } \\
\text { myristic acid (106) }\end{array}$ & \\
\hline & $\mathrm{B}, \mathrm{L}$ & $\begin{array}{l}\text { M, E, EE, } \\
\text { EA, W }\end{array}$ & Glycosides & $\begin{array}{c}\text { Geniposidic acid (107), 2'-cinnamoyl-mussaenosidic acid (108), mussaenoside (109), } \\
\text { 2'-cinnamoyl-mussaenoside (110), 10-O-5-phenyl-2,4-pentadienoyl-geniposide (111), } \\
\text { 7-O-5-phenyl-2,4-pentadienoyl-8-epiloganin (112), 10-O-(E-cinnamoyl)-geniposidic acid } \\
\text { (113), 2'-O-(2E,4E-5-phenylpenta-2,4-dienoyl)mussaenosidic acid (114), marinoids A-E } \\
\text { (115-119), verbascoside (120), isoverbascoside (121), derhamnosylverbascosid (122), } \\
\text { 11-hydroxy- 8,11,13-abietatriene 12-O- } \beta \text {-xylopyranoside (123), lyoniresinol } \\
\text { 9'-O- } \beta \text {-D-glucopyranoside (124) }\end{array}$ & \\
\hline & $\mathrm{L}$ & $\mathrm{CE}$ & $\begin{array}{l}\text { Alkaloids, flavonoids, terpenoids, } \\
\text { phenolics, saponins, amino acid }\end{array}$ & NI & [114] \\
\hline $\begin{array}{l}\text { icennia germinans (L.) } \\
\text { L. }\end{array}$ & $\mathrm{L}$ & NI & Glycosides & NI & {$[7,161,162]$} \\
\hline
\end{tabular}


Table 8. Cont.

\begin{tabular}{|c|c|c|c|c|c|}
\hline Species & Plant Part & Extract & Phytochemical Class & Constituent & Reference \\
\hline \multirow{11}{*}{ Avicennia officinalis $\mathrm{L}$. } & $\mathrm{L}$ & Me & $\begin{array}{l}\text { Alkaloid, reducing sugar, tannins, } \\
\text { gums, flavonoids, steroid }\end{array}$ & NI & [116] \\
\hline & $\mathrm{L}$ & CE & $\begin{array}{l}\text { Alkaloid, flavonoid, terpenoids, } \\
\text { phenolics, tannins, sterols, glycosides }\end{array}$ & NI & [114] \\
\hline & $\mathrm{L}$ & $\mathrm{Me}$ & Pentacyclic triterpenoids & Lupeol (48), betulin (99), betulinaldehyde (125), betulinic acid (98), $\beta$-sitosterol (54) & \multirow[b]{2}{*}{ [79] } \\
\hline & $\mathrm{L}$ & $\mathrm{Me}$ & $\begin{array}{l}\text { Glycosides, flavonoids, alkaloids, } \\
\text { steroids, tannins, wax esters }\end{array}$ & NI & \\
\hline & $\mathrm{L}$ & NI & Flavonoid & Velutin (126) & \multirow{6}{*}{ [7] } \\
\hline & $\mathrm{L}$ & NI & Naphthalene derivatives & Avicenol C (127) & \\
\hline & $\mathrm{L}$ & NI & Tannins & Catechin (128), chlorogenic acid (129), gallic acid (130), elagic acid (131) & \\
\hline & $\mathrm{L}$ & NI & Steroids & $\begin{array}{l}\beta \text {-sitosterol (54), stigmasterol (53), cholesterol (51), campesterol (52), stigmast-7-en-3 } \beta \text {-ol } \\
\text { (132) }\end{array}$ & \\
\hline & $\mathrm{L}$ & NI & Terpenoids & $\begin{array}{c}\text { Taraxerol (96), saraxerone (97), setulinic acid (98), setulin (99), betulinaldehyde (125), } \\
\text { 3-amyrin (47), rhizophorins A-B (133-134) ent-13S-2,3-seco-14-labden-2,8-olide-3-oic acid } \\
\text { (135), ribenone (136), ent-16-hydroxy-3-oxo-13-epi-manoyl oxide (137), ent-15- } \\
\text { hydroxy-labda-8, 13E-dien-3-one (138), ent-3a,15-dihydroxylabda-8,13E-diene (139), } \\
\text { excoecarin A(140), ent-beyerane (141) }\end{array}$ & \\
\hline & $\mathrm{L}$ & NI & Glycosides & $\begin{array}{c}\text { 7-O-trans-cinnamoyl-4-epilogenin (142), geniposidic acid (107), } \\
\text { 2'-cinnamoyl-mussaenosidic acid (108), 10-O-5-phenyl-2,4-pentadienoyl-geniposide (111), } \\
\text { 7-O-cinnamoyl-8-epiloganic acid sodium salt (143), 8-O-cinnamoylmussaenosidic acid } \\
\text { (144), officinosidic acid (145), loganin C (146), }\end{array}$ & \\
\hline & $\mathrm{L}$ & $\mathrm{E}$ & $\begin{array}{l}\text { Carbohydrate, reducing sugar, } \\
\text { combined reducing sugar, } \\
\text { glycosides, tannins, alkaloids, proteins, } \\
\text { terpenoids and flavonoids }\end{array}$ & NI & [118] \\
\hline \multirow[t]{2}{*}{$\begin{array}{l}\text { Bruguiera cylindrica (L.) } \\
\quad \text { Blume }\end{array}$} & Fr & NI & Pentacyclic triterpenoids esters & 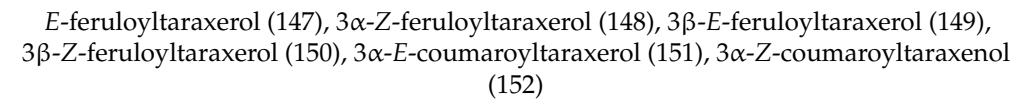 & [163] \\
\hline & $\mathrm{L}$ & NI & $\begin{array}{l}\text { Tannins, saponins, alkaloids, } \\
\text { triterpenoids, anthraquinone, } \\
\text { flavonoids }\end{array}$ & NI & [120] \\
\hline $\begin{array}{l}\text { Bruguiera parviflora } \\
\text { (Roxb.) Wight \& Arn. ex } \\
\text { Griff. }\end{array}$ & B & NI & Phenolic compounds & NI & [101] \\
\hline $\begin{array}{l}\text { Bruguiera conjugata }(\mathrm{L} .) \\
\text { Merr. }\end{array}$ & St, B & NI & Sulfur containing alkaloids & NI & [88] \\
\hline
\end{tabular}


Table 8. Cont.

\begin{tabular}{|c|c|c|c|c|c|}
\hline Species & Plant Part & Extract & Phytochemical Class & Constituent & Reference \\
\hline Bruguiera rumphii Blume & $\mathrm{B}, \mathrm{L}$ & NI & Tannins, triterpenes & $\mathrm{NI}$ & [88] \\
\hline $\begin{array}{l}\text { Bruguiera sexangula } \\
\text { (Lour.) Poir. }\end{array}$ & B & NI & Phenolics, steroids, alkaloids, tannins & NI & [88] \\
\hline \multirow{10}{*}{$\begin{array}{l}\text { Bruguiera gymnorhiza }(\mathrm{L} .) \\
\text { Lam }\end{array}$} & $\mathrm{L}$ & $\mathrm{Me}$ & $\begin{array}{l}\text { Flavonoids, saponins, reducing sugars, } \\
\text { tannins, gums }\end{array}$ & NI & [81] \\
\hline & F & NI & Dammarane triterpenes & Bruguierol A-C (153-155), bruguiesulfurol (156), brugierol (157), isobrugierol (158) & \multirow{6}{*}{ [164] } \\
\hline & St & NI & Pimaren diterpenes & $\begin{array}{l}\text { ent-8(14)-pimarene-15R, 16-diol (159), ent-8(14)-pimarene-1alpha, 15R,16-triol (160), } \\
\text { isopimar-7-ene-15S,16-diol (161), (-)-1 } \beta, 15(R) \text {-ent-pimar-8(14)-en-1,15,16-triol (162) }\end{array}$ & \\
\hline & & & Aromatic compounds & 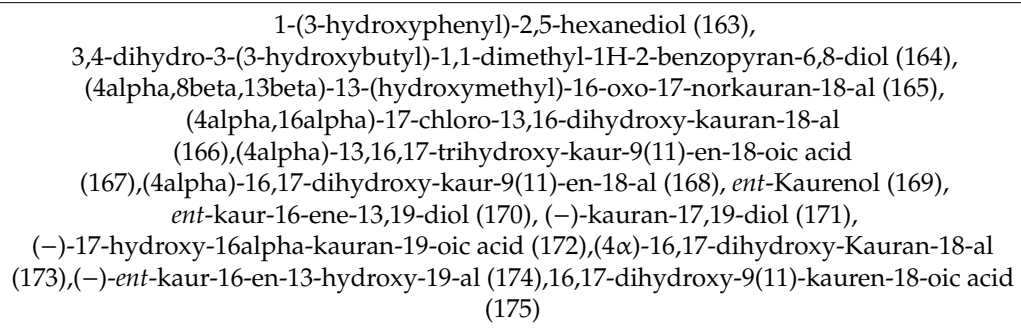 & \\
\hline & WP & NI & Gibberellin & Gymnorrhizol (176), gibberellin A3 (177), A4 (178), A7 (179) & \\
\hline & $\mathrm{L}$ & NI & Sterols & Cholesterol (51), campesterol (52), stigmasterol (53), 28-isofucosterol (56) & \\
\hline & $\mathrm{R}$ & NI & Diterpenoids & $\begin{array}{c}\text { Steviol (180), (-)-ent-kaur-16-en-13-hydroxy-19-al (174), 15(S)-isopimar-7-en-15,16-diol } \\
\text { (181), ent-kaur-16-en-13,19-diol (177), } \\
\text { methyl-ent-kaur-9(11)-en-13,17-epoxy-16-hydroxy-19-oate (182), apiculol } \\
\text { (1-hydroxy-epimanoyl oxide) (183) }\end{array}$ & \\
\hline & NI & NI & NI & Gymnorrhizol (176) & [165] \\
\hline & $\mathrm{R}$ & $\mathrm{Me}$ & $\begin{array}{l}\text { Gums, flavonoids, saponins, reducing } \\
\text { sugar, tannins }\end{array}$ & NI & [152] \\
\hline & $\mathrm{Fr}$ & NI & Anthocyanins, catechins, diterpenes & NI & [88] \\
\hline $\begin{array}{l}\text { Ceriops roxburghiana } \\
\text { Arn. }\end{array}$ & WP & NI & Gibberellins, procyanidins & NI & [88] \\
\hline
\end{tabular}


Table 8. Cont.

\begin{tabular}{|c|c|c|c|c|c|}
\hline Species & Plant Part & Extract & Phytochemical Class & Constituent & Reference \\
\hline \multirow{9}{*}{$\begin{array}{l}\text { Ceriops decandra (Griff.) } \\
\text { W. Theob. }\end{array}$} & B, Fr, L & NI & Polyphenols, tannins, triterpenes & NI & [88] \\
\hline & $\mathrm{L}$ & $\mathrm{B}, \mathrm{E}$ & $\begin{array}{l}\text { Protein, coumarin, phenols, flavonoids, } \\
\text { saponins, glycosides, alkaloids, } \\
\text { terpenoids, tannins }\end{array}$ & NI & {$[80,166]$} \\
\hline & & & Diterpenoids & Ceriopsin F, G $(184,185)$ & \\
\hline & $\mathrm{R}$ & EA & NI & $\begin{array}{c}\text { ent-13-hydroxy-16-kauren-19-oic acid (186), methyl } \\
\text { ent-16 } \beta, 17 \text {-dihydroxy-9(11)-kauren-19-oat (187), } \\
\text { ent-16 } \beta, 17 \text {-dihydroxy-9(11)-kauren-19-oic acid (188), ent-16-oxobeyeran-19-oic acid (189), } \\
\text { 8,15R-epoxypimaran-16-ol (190) }\end{array}$ & [167] \\
\hline & NI & NI & $\begin{array}{l}\text { Alkaloids, flavonoids, phenols, } \\
\text { saponins, steroids, tannins, terpenoids }\end{array}$ & NI & [168] \\
\hline & $\mathrm{L}$ & $\mathrm{H}$ & $\begin{array}{l}\text { Carbohydrates, free reducing sugars, } \\
\text { tannins, steroids, cardiac glycosides, } \\
\text { terpenoids, flavonoids }\end{array}$ & NI & \multirow{4}{*}{ [169] } \\
\hline & $\mathrm{L}$ & $\mathrm{C}$ & $\begin{array}{c}\text { Carbohydrates, combined reducing } \\
\text { sugars, steroids, cardiac glycosides, } \\
\text { terpenoids }\end{array}$ & NI & \\
\hline & $\mathrm{L}$ & Ac & $\begin{array}{l}\text { Carbohydrates, monosaccharides, } \\
\text { combined reducing sugars, tannins, } \\
\text { free anthraquinones, flavonoids, } \\
\text { soluble starch, alkaloids }\end{array}$ & NI & \\
\hline & $\mathrm{L}$ & $\mathrm{Me}$ & $\begin{array}{l}\text { Carbohydrates, monosaccharides, } \\
\text { combined reducing sugars, tannins, } \\
\text { free anthraquinones, flavonoids, } \\
\text { soluble starch }\end{array}$ & NI & \\
\hline \multirow{6}{*}{$\begin{array}{l}\text { Ceriops tagal (Perr.) C. B. } \\
\text { Rob. }\end{array}$} & B & NI & $\begin{array}{l}\text { Inositols, steroids, polyphenols, } \\
\text { tannins }\end{array}$ & NI & [88] \\
\hline & $\mathrm{R}$ & NI & Dimeric diterpenoids & 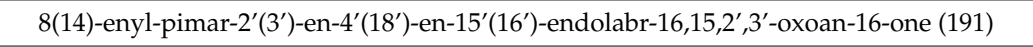 & \multirow{2}{*}{ [170] } \\
\hline & $\mathrm{R}$ & NI & Terpenoids & $\begin{array}{l}\text { Tagalsin C (192), Tagalsin I (193), lup-20(29)-ene-3ß,28-diol (194), } \\
\text { 3-oxolup-20(29)-en-28-oic acid (195), 28-hydroxylup-20(29)-en-3-one (196) }\end{array}$ & \\
\hline & $\mathrm{AP}$ & E & Dolabranes & Tagalsin V (197), Tagalsin W (198) & \multirow[b]{2}{*}{ [171] } \\
\hline & $\mathrm{AP}$ & $\mathrm{E}$ & Terpenes & $\begin{array}{c}\text { ent-5 } \alpha, 3,15 \text {-dioxodolabr-1,4(18)-diene-2,16-diol (199), tagalsin S (200), tagalsin P (201), } \\
\text { ent-5 } \alpha, 2,15 \text {-dioxodolabr-3-ene-3,16-diol (202), ent-8(14)-pimarene-15R,16-diol (165), } \\
\text { 3a-lup-20(29)-ene-3,28-diol (203) }\end{array}$ & \\
\hline & St, Tw & E & NI & Tagalsin H (204) & [172] \\
\hline
\end{tabular}


Table 8. Cont.

\begin{tabular}{|c|c|c|c|c|c|}
\hline Species & Plant Part & Extract & Phytochemical Class & Constituent & Reference \\
\hline \multirow{5}{*}{$\begin{array}{l}\text { Ceriops tagal (Perr.) C. B. } \\
\text { Rob. }\end{array}$} & $\mathrm{AP}$ & $\mathrm{E}$ & Lupane-type triterpenes & 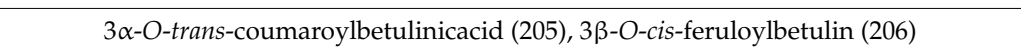 & \\
\hline & $\mathrm{AP}$ & $\mathrm{E}$ & Triterpenes & 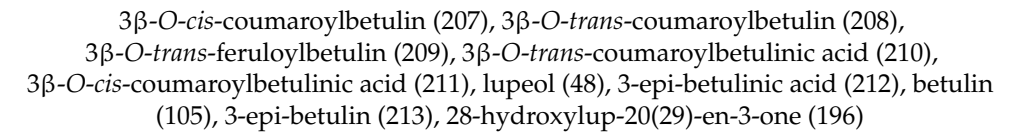 & [29] \\
\hline & St, Tw & NI & Dolabranes & Tagalsin P (201), Q (214), R (215), S (200), T (216), U (217) & \multirow{3}{*}{ [173] } \\
\hline & St, Tw & NI & Pimarane & NI & \\
\hline & St, Tw & NI & Abietane & NI & \\
\hline \multirow{12}{*}{ Excoecaria agallocha $\mathrm{L}$. } & St, Tw & NI & ent-kaurane diterpenoids & Agallochaol K (218), L (219), M (220), N (221), O (222), P (223) & \multirow{3}{*}{ [174] } \\
\hline & St, Tw & NI & Atisane-type diterpenoid & Agallochaol Q (224) & \\
\hline & St, Tw & NI & Diterpenoids & NI & \\
\hline & B & NI & ent-isopimarane-type diterpenoid & NI & [175] \\
\hline & NI & NI & Diterpenoids & Excoecarins D, E, K (225-227) & [176] \\
\hline & WP & NI & $\begin{array}{l}\text { Alkaloids, tannins, phorbol esters, } \\
\text { polyphenols }\end{array}$ & NI & [88] \\
\hline & NI & NI & Diterpenoids & 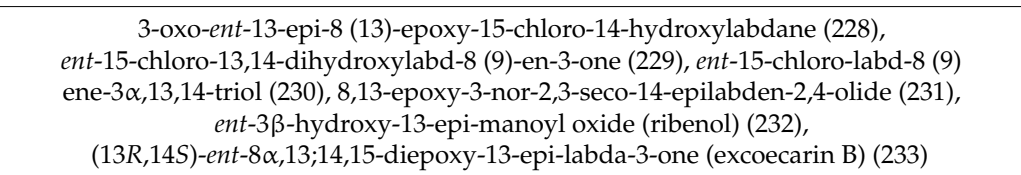 & \multirow{6}{*}{ [50] } \\
\hline & NI & NI & Triterpenoids & $\begin{array}{l}\text { 3ß-(2E,4E)-5-oxo-decadienoyloxy-olean-12-ene (34), } \beta \text {-amyrin acetate (235), Taraxerone } \\
\text { (197), 3-epitaraxerol (236), taraxerol (196), 3-epilupeol (237), acetylaleuritolic acid (238) }\end{array}$ & \\
\hline & NI & NI & Flavonoids & $\begin{array}{c}2^{\prime}, 4^{\prime}, 6^{\prime}, 4 \text {-tetramethoxychalcone (239), 3,5,7,3',5'-pentahydroxy-2R,3R-flavanonol } \\
\text { 3-O- } \alpha \text {-L-rhamnopyranoside (240) }\end{array}$ & \\
\hline & NI & NI & Alkaloid & 2,4-dimethoxy-3- $\psi, \psi$-dimethylallyl-trans-cinnamoylpiperidide (241) & \\
\hline & NI & NI & Sterols & $\beta$-sitostenone (242), (24R)-24-ethylcholesta-4,22-dien-3-one (243) & \\
\hline & NI & NI & Tannin & 3,4,5-trimethoxyphenol 1-O-ß-D-(6-galloyl)-glucopyranoside (244) & \\
\hline
\end{tabular}


Table 8. Cont

\begin{tabular}{|c|c|c|c|c|c|}
\hline Species & Plant Part & Extract & Phytochemical Class & Constituent & Reference \\
\hline \multirow{5}{*}{$\begin{array}{l}\text { Heritiera fomes } \\
\text { Buch.-Ham. }\end{array}$} & $\mathrm{L}$ & E & $\begin{array}{l}\text { Flavonoids, tannins, alkaloids, } \\
\text { terpenoids, saponins }\end{array}$ & NI & [89] \\
\hline & $\mathrm{L}$ & NI & $\begin{array}{l}\text { Saponins, alkaloids, glycosides, } \\
\text { steroids, flavonoids, gum, phytosterols, } \\
\text { reducing sugars }\end{array}$ & NI & \multirow[t]{2}{*}{ [51] } \\
\hline & $\mathrm{B}$ & $\mathrm{NI}$ & Proanthocyanidins & $\mathrm{NI}$ & \\
\hline & $\mathrm{Sb}$ & $\mathrm{Aq}$ & $\begin{array}{c}\text { Alkaloids, cardiac glycoside, } \\
\text { anthraquinone glycoside, tannin, } \\
\text { steroids, saponins, flavonoids, gums } \\
\text { and mucilages, carbohydrates, proteins } \\
\text { and amino acids, terpenoid }\end{array}$ & NI & \multirow[t]{2}{*}{ [90] } \\
\hline & $\mathrm{Sb}$ & Ac & $\begin{array}{l}\text { Alkaloids, cardiac glycoside, } \\
\text { anthraquinone glycoside, flavonoids, } \\
\text { carbohydrates, proteins and amino } \\
\text { acids, terpenoid }\end{array}$ & NI & \\
\hline \multirow[t]{2}{*}{ Heritiera littoralis Aiton } & $\mathrm{L}$ & E & Flavonoids & $\begin{array}{l}\text { 3,5,7-trihydroxychromone-3-O- } \alpha \text {-L-rhamnopyranoside (245), } \\
\text { quercetin-3-O- } \alpha \text {-L-rhamnopyranoside (246), } \\
(2 R, 3 R) \text {-dihydroquercetin-3-O- } \alpha \text {-L-rhamnopyranoside (247), } \\
\text { kaempferol-3-O- } \alpha \text {-L-rhamnopyranoside (248) }\end{array}$ & [92] \\
\hline & St, B, Fr, L & NI & $\begin{array}{l}\text { Alkaloids, tannins, polyphenols, } \\
\text { saponins }\end{array}$ & NI & [88] \\
\hline \multirow{3}{*}{$\begin{array}{c}\text { Lumnitzera racemosa } \\
\text { Willd. }\end{array}$} & $\mathrm{L}$ & $\mathrm{Aq}$ & $\begin{array}{l}\text { Phenols, flavonoids, alkaloids, } \\
\text { terpenoids, sterols, tannins, } \\
\text { carbohydrates, cardiac glycosides, } \\
\text { saponins, quinines }\end{array}$ & NI & [127] \\
\hline & $\mathrm{Tw}$ & $\mathrm{Me}$ & Flavonoid, quercetin, myricetin & $\mathrm{NI}$ & [177] \\
\hline & St & $\mathrm{CH}_{2} \mathrm{Cl}_{2}: \mathrm{Me}$ & Aromatic ester & $\mathrm{NI}$ & [167] \\
\hline \multirow{4}{*}{$\begin{array}{l}\text { Kandelia candel (L.) } \\
\text { Druce }\end{array}$} & WP & NI & $\begin{array}{l}\text { Alkaloids, tannins, saponins, } \\
\text { polyphenols }\end{array}$ & NI & [88] \\
\hline & $\mathrm{L}$ & $\mathrm{C}$ & $\begin{array}{l}\text { Carbohydrate, alkaloid, flavonoid, } \\
\text { tannin, phenol }\end{array}$ & NI & \multirow{3}{*}{ [178] } \\
\hline & $\mathrm{L}$ & EA & Carbohydrate, alkaloid, glycoside & NI & \\
\hline & $\mathrm{L}$ & E & Carbohydrate, protein, amino acid & $\mathrm{NI}$ & \\
\hline Kandelia rheedii & $\mathrm{B}, \mathrm{Fr}, \mathrm{L}$ & NI & Steroids, triterpenoids & NI & [88] \\
\hline
\end{tabular}


Table 8. Cont.

\begin{tabular}{|c|c|c|c|c|c|}
\hline Species & Plant Part & Extract & Phytochemical Class & Constituent & Reference \\
\hline \multirow{5}{*}{ Nypa fruticans Wurmb } & NI & $\mathrm{Aq}$ & NI & $\begin{array}{l}\text { Acetic acid (249), 2,3-butanediol (250), 1-(2-butoxyethoxy)-ethanol (251), } \\
\text { 5-bromo-2-hydroxybenzaldehyde (252), (4-aminophenyl)-phenylmethanone (253) }\end{array}$ & [96] \\
\hline & Fr & $\mathrm{Aq}$ & NI & $\begin{array}{c}\text { Gallic acid (130), protocatechuic acid (54), 4-hydroxybenzoic acid (255), chlorogenic acid } \\
\text { (129), rutin (256), cinnamic acid (257), quercetin (12), kaempferol (79) }\end{array}$ & [179] \\
\hline & $\mathrm{L}$ & E & $\begin{array}{l}\text { Alkaloids, cardiac glycosides, } \\
\text { anthranoids, polyphenols, flavonoid }\end{array}$ & . & \multirow{2}{*}{ [180] } \\
\hline & $\mathrm{Hu}$ & $\mathrm{E}$ & $\begin{array}{l}\text { Alkaloids, phlobotannins, anthranoids, } \\
\text { polyphenols, saponins }\end{array}$ & NI & \\
\hline & $\mathrm{L}, \mathrm{Fr}$ & NI & NI & Acetic acid (249), & [88] \\
\hline $\begin{array}{l}\text { Pelliciera rhizophorae } \\
\text { Planch. \& Triana }\end{array}$ & $\mathrm{L}$ & $\mathrm{CE}$ & NI & $\begin{array}{c}\alpha \text {-amyrin (46), } \beta \text {-amyrin (47), ursolic acid (50), oleanolic acid (49), betulinic acid (98), } \\
\text { brugierol (157), iso-brugierol (158), kaempferol (79), quercetin (12) }\end{array}$ & [44] \\
\hline \multirow{3}{*}{$\begin{array}{l}\text { Rhizophora apiculata } \\
\text { Blume }\end{array}$} & $\mathrm{B}, \mathrm{F}, \mathrm{Fr}, \mathrm{L}$ & NI & $\begin{array}{l}\text { Aliphatic alcohols, hydrolysable } \\
\text { tannins, steroids, triterpenoids, } \\
\text { phenolic compounds }\end{array}$ & NI & {$[88]$} \\
\hline & $\mathrm{Tw}, \mathrm{L}, \mathrm{B}$ & NI & NI & 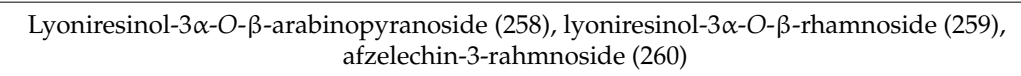 & [132] \\
\hline & NI & CE & $\begin{array}{l}\text { Alcohols, ketones, furan and pyran } \\
\text { derivatives, guaicol and derivatives, } \\
\text { phenol and derivatives, syringol and } \\
\text { derivatives, pyrocatechol, alkyl aryl } \\
\text { ether, nitrogenated compounds, } \\
\text { carbohydrate derivatives, carbohydrate } \\
\text { derivatives }\end{array}$ & NI & [134] \\
\hline \multirow{3}{*}{$\begin{array}{l}\text { Rhizophora mucronata } \\
\text { Lam. }\end{array}$} & B, Fr, F, R & NI & $\begin{array}{l}\text { Alkaloids, tannins, gibberellins, } \\
\text { inositol saponins, lipids }\end{array}$ & NI & {$[88]$} \\
\hline & NI & NI & $\begin{array}{c}\text { Proteins, minerals, carotenoids, } \\
\text { hydrolysable tannins, lipids, } \\
\text { polysaccharides, steroids, triterpenes, } \\
\text { condensed tannins, procyanidins, } \\
\text { anthocyanidins, alkaloids, } \\
\text { carbohydrates, chlorophyll, } \\
\text { gibberellins, flavonoids, inositols, } \\
\text { polyphenols, saponins }\end{array}$ & NI & [181] \\
\hline & $\mathrm{L}$ & $\mathrm{C}$ & Oleanenes & 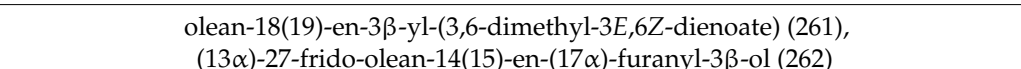 & [137] \\
\hline
\end{tabular}


Table 8. Cont.

\begin{tabular}{|c|c|c|c|c|c|}
\hline Species & Plant Part & Extract & Phytochemical Class & Constituent & Reference \\
\hline \multirow{6}{*}{$\begin{array}{l}\text { Rhizophora mucronata } \\
\text { Lam. }\end{array}$} & L, B, Fr, F & $\mathrm{Me}$ & $\begin{array}{l}\text { Alkaloid, tannin, saponin, phenolic, } \\
\text { flavonoid, terpenoid, steroid, } \\
\text { glycosides }\end{array}$ & NI & \multirow{2}{*}{ [60] } \\
\hline & $\mathrm{R}$ & $\mathrm{Me}$ & $\begin{array}{l}\text { Alkaloid, tannin, saponin, steroids, } \\
\text { glycosides }\end{array}$ & NI & \\
\hline & $\mathrm{R}$ & EA & Diterpenoids & Rhizophorin A (133), rhizophorin B (134), rhizophorin C-E (263-264) & [167] \\
\hline & B & NI & Lupeol, quercetin, caffeic acid & NI & [154] \\
\hline & $\mathrm{L}, \mathrm{F}$ & $\mathrm{H}$ & $\begin{array}{l}\text { Tannin, saponin, terpenoid, alkaloid, } \\
\text { flavonoid }\end{array}$ & NI & [142] \\
\hline & $\mathrm{AP}$ & $\mathrm{CE}$ & Ethanone & 1-(2-hydroxy-5-methylphenyl) (265) & [143] \\
\hline \multirow[t]{2}{*}{ Rhizophora conjugata $\mathrm{L}$. } & B & NI & $\begin{array}{l}\text { Anthocyanins, tannins, steroids, } \\
\text { triterpenoids }\end{array}$ & NI & [88] \\
\hline & St & $\mathrm{E}$ & NI & $\begin{array}{l}\text { Lyoniresinol-3 } \alpha \text {-O- } \beta \text {-arabinopyranoside (258), lyoniresinol-3 } \alpha \text {-O- } \beta \text {-rhamnoside (259), } \\
\text { afzelechin-3-O-L-rhamno-pyranoside (266) }\end{array}$ & [132] \\
\hline \multirow{5}{*}{ Rhizophora mangle L. } & B, L & NI & Tannins, triterpenes & NI & [88] \\
\hline & $\mathrm{L}$ & NI & $\begin{array}{l}\text { Flavonoid glycosides, quercetin, } \\
\text { myricetin, kaempferol diglycosides }\end{array}$ & NI & [182] \\
\hline & Co & E & NI & $\begin{array}{l}\text { Cinchonain Ia (267), Ib (268), catechin-3-O-rhamnopyranoside (269), lyoniside (270), } \\
\text { nudiposide (271) }\end{array}$ & [183] \\
\hline & \multirow{2}{*}{$\mathrm{R}$} & \multirow{2}{*}{$\mathrm{H}$} & Diterpenes & Manool (272), jhanol (273), steviol (180) & \multirow{2}{*}{ [184] } \\
\hline & & & Benzaldehyde & p-oxy-2-ethylhexyl benzaldehyde (274) & \\
\hline $\begin{array}{l}\text { Rhizophora racemosa G. } \\
\text { Mey. }\end{array}$ & F, L & NI & Tannins, steroids & NI & [88] \\
\hline \multirow{4}{*}{ Rhizophora stylosa Griff. } & St, Tw & $\mathrm{CE}$ & Acetylated flavonol & 3,7-O-diacetyl (-)-epicatechin (275) & \\
\hline & St, Tw & $\mathrm{CE}$ & Flavonol derivatives & $\begin{array}{l}\text { (-)-epicatechin (276), 3-O-acetyl (-)-epicatechin (277), 3,3' , ,' }, 5,7-\mathrm{O}-\text { pentaacetyl } \\
\text { (-)-epicatechin (278), (+)-afzelechin (279), cinchonain Ib (268), proanthocyanidin B2 (280) }\end{array}$ & [174] \\
\hline & L, R, Se & NI & Inositols, steroids & $\mathrm{NI}$ & {$[88]$} \\
\hline & $\mathrm{L}, \mathrm{F}$ & $\mathrm{H}$ & Tannins, saponin, terpenoid, flavonoid & NI & [142] \\
\hline \multirow[t]{2}{*}{$\begin{array}{l}\text { Xylocarpus granatum } \mathrm{J} \text {. } \\
\text { Koenig }\end{array}$} & Se & NI & NI & $\begin{array}{c}\text { 2,3-dideacetylxyloccensin S (281), } 30 \text { deacetylxyloccensin W (282), } \\
\text { 7-hydroxy-21b-methoxy-3-oxo-24,25,26,27-tetranortirucalla-1,14-diene-23(21)-lactone } \\
\text { (283) }\end{array}$ & [185] \\
\hline & NI & NI & NI & $\begin{array}{l}\text { Xyloccensin O (284), xyloccensin P (285), gedunin }(286) \text {, catechin }(141),(-) \text { epicatechin } \\
(276) \text {, procyanidin B1 (287), procyanidin trimer (288), procyanidin pentamer (289) }\end{array}$ & [147] \\
\hline
\end{tabular}

$\mathrm{L}=$ Leaf, $\mathrm{NI}=$ Not indicated, $\mathrm{H}=$ Hexane, $\mathrm{Hu}=$ Husk, $\mathrm{Me}=$ Methanol, $\mathrm{R}=$ Root, $\mathrm{Se}=$ Seed, $\mathrm{St}=\mathrm{Stem}, \mathrm{Sb}=\mathrm{Stem}$ bark, Tw $=$ Twig, WP $=\mathrm{Whole} \mathrm{plant.}$ 


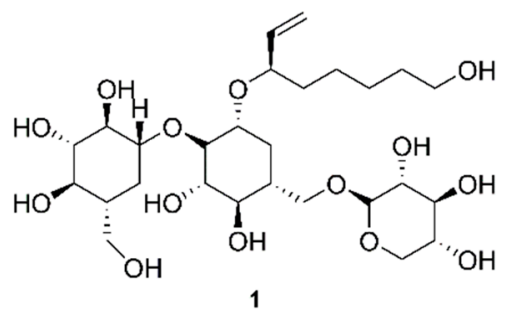

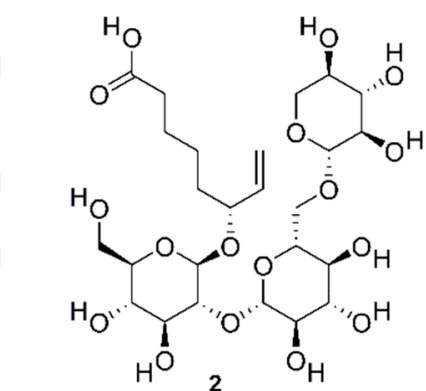<smiles>O=c1[nH]c2ccccc2o1</smiles><smiles>O=C1COc2ccccc2N1</smiles><smiles>COc1ccc2c(c1)O[C@H](OOC1OC(CO)[C@@H](O)C(O)C1O)C(=O)N2OC</smiles><smiles>O=c1[nH]c2c(OC3OC(CO)C(O)C(O)[C@H]3O)cccc2o1</smiles><smiles>O=c1[nH]c2cc(-c3ccc4oc(=O)[nH]c4c3)ccc2o1</smiles><smiles></smiles><smiles>O=C1Nc2ccccc2OC1CC1OC2(O)OC(O)C(O)C1O2</smiles><smiles>O=C1C(=O)N(O)c2ccccc2C1COC1OC(CO)[C@H](O)C(O)C1O</smiles><smiles>COC(=O)c1ccc[n+](C)c1</smiles><smiles>CCC1=Cc2c(O)cncc2C(C)N1</smiles> 


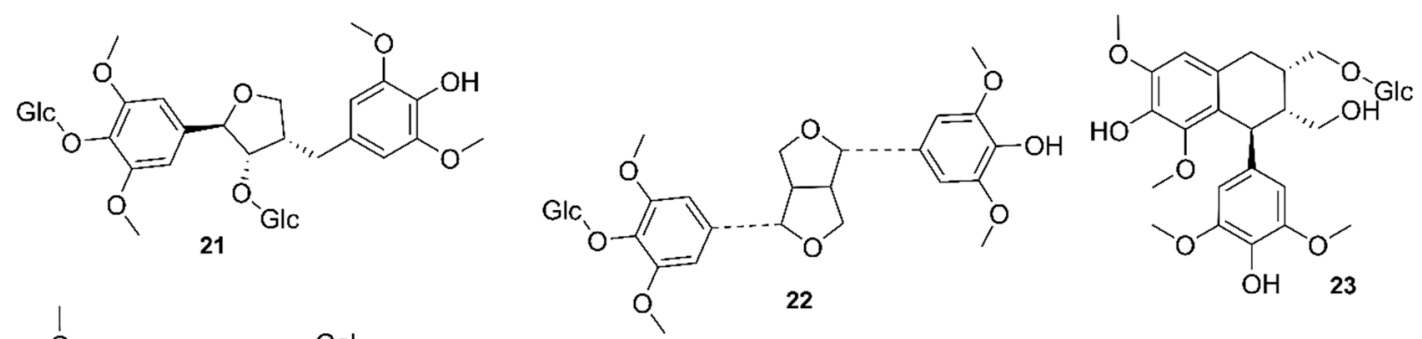<smiles>COCC1Cc2cc(OC)c(O)c(OC)c2C(c2cc(OC)c(O)c(OC)c2OC)C1COC</smiles>

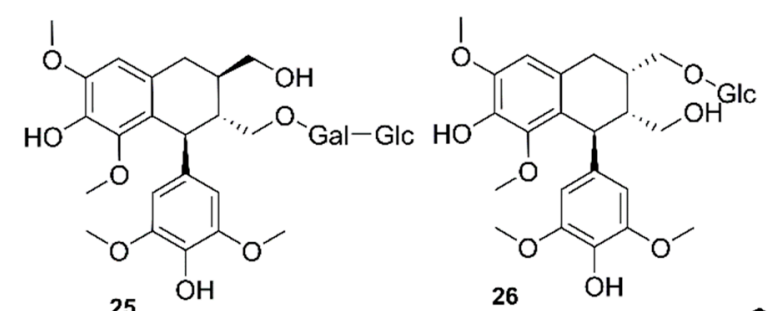

25<smiles>O=C(O)/C=C/c1ccc(OC2OC(CO)C(O)C(O)C2O)cc1</smiles>

HO<smiles>CC1=CC(=O)CC(C)(C)C1/C=C/C(C)OC1OC(CO)[C@H](O)C(O)C1O</smiles>

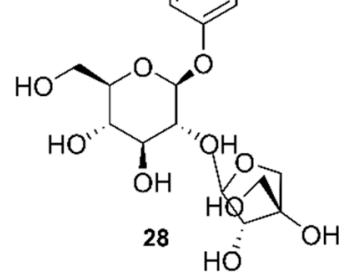

27<smiles>CC1=CC(=O)C[C@H](C)[C]1/C=C/C(C)Cl</smiles>

30<smiles>CC1=C(/C=C/C(C)O)C(C)(C)CC(OC2C(O)C(O)[C@@H](O)C(CO)C2O)C1C</smiles>

31<smiles>CC=C(COC1OC(CO)C(O)C(O)C1O)OC</smiles><smiles>CC(=O)/C=C/C(C)C(C)CC(CC(C)C)OC1OC(CO)C(O)C(O)C1O</smiles>

34<smiles>OCC1OC(OC2C(OCCc3ccccc3)OC(CO)[C@H](O)C2O)C(O)C(O)[C@@H]1O</smiles><smiles>OCC1OC(OCCc2ccccc2)C(O)C(O)[C@@H]1O</smiles><smiles>CC1C(OC(=O)CCc2ccc(O)c(O)c2)C(OC2OC(C)[C@@H](O)C(O)O2)C(O)[C@@H](O)C1O</smiles><smiles>C[C@@H]1OC(OC2CCOC(CO)C2O)[C@H](O)[C@@H](O)[C@H]1O</smiles><smiles>COc1cc(CCOC2OC(CO)[C@@H](O)C(OC3O[C@H](C)[C@@H](O)[C@H](O)[C@H]3O)C2O)ccc1O</smiles>

Figure 12. Chemical structures of compounds 21-39 isolated from mangrove species. 


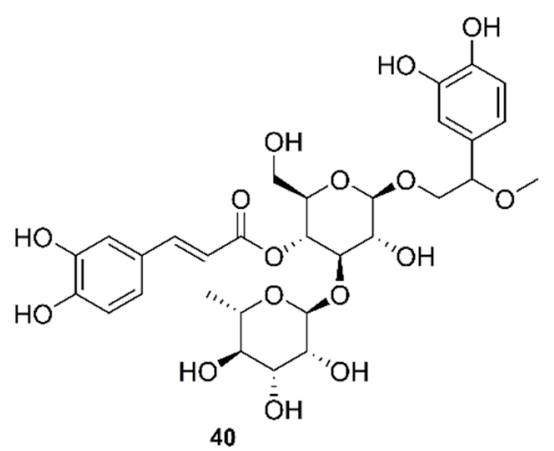<smiles>C[C@H]1O[C@H](O[C@@H]2[C@@H](O)[C@H](OCCc3ccc(O)c(O)c3)O[C@H](CO)[C@H]2OC(=O)/C=C/c2ccc(O)c(O)c2)[C@H](O)[C@H](O)[C@@H]1O</smiles>

43

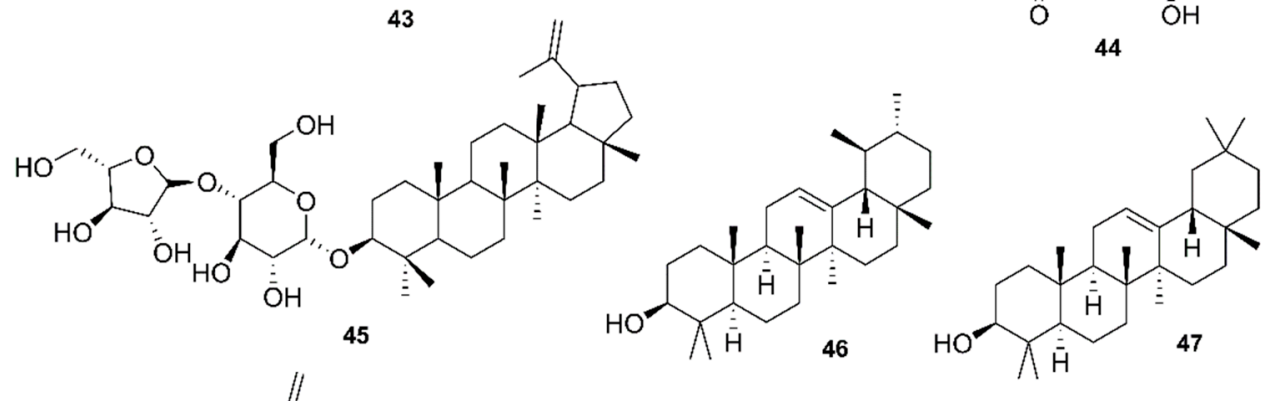

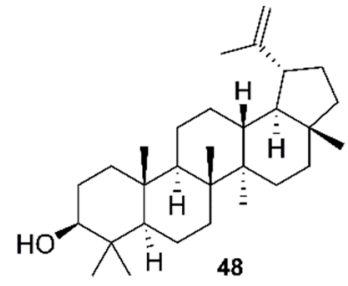<smiles>CC1(C)CC[C@]2(C(=O)O)CC[C@H]3C(=CCC4[C@@]5(C)CC[C@H](O)C(C)(C)C5CC[C@]43C)C2C1</smiles>

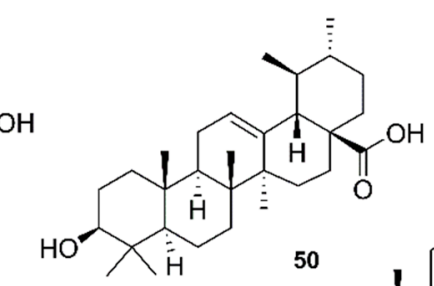<smiles>CCI</smiles><smiles>CCC(C)C(C)CC[C@H](C)[C@H]1CCC2C3=CCC4CC(O)CCC4(C)[C@H]3CC[C@]21C</smiles>

Figure 13. Chemical structures of compounds $\mathbf{4 0 - 5 5}$ isolated from mangrove species. 


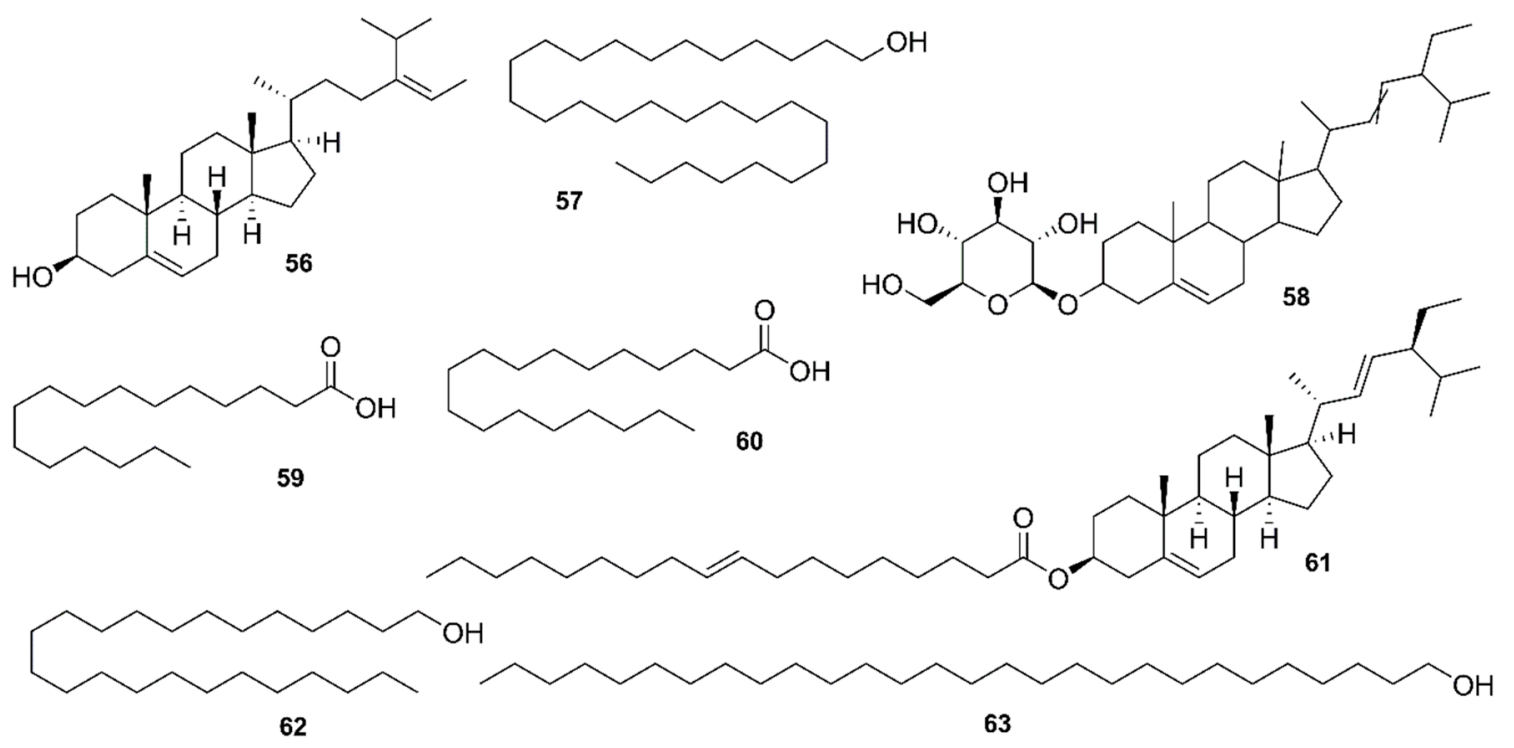

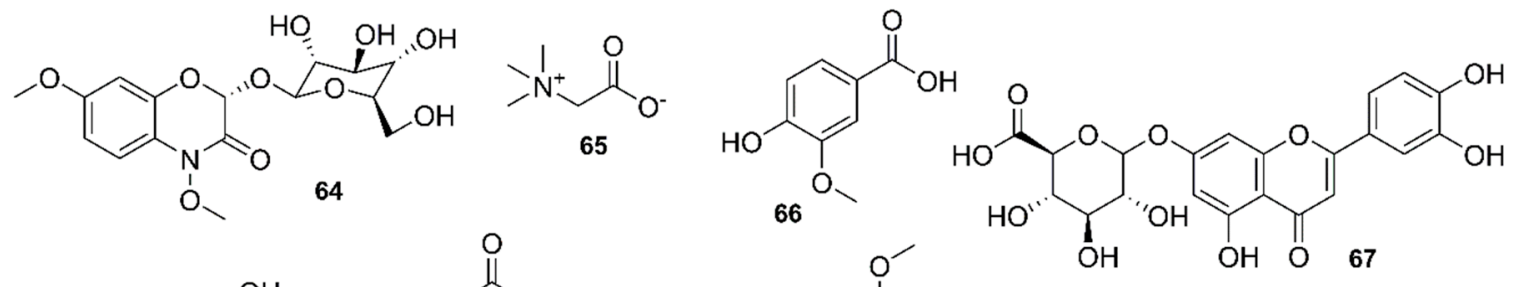<smiles>COc1cc(C(=O)OC2OC(CO)[C@@H](O)C(O)C2O)cc(OC)c1O</smiles>

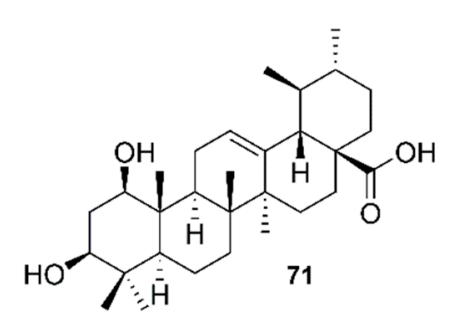<smiles>[Y6]CC[C@H](C)[C@@H](C)CO</smiles><smiles>COc1cc(O)c2c(=O)cc(-c3ccc(O)c(O)c3)oc2c1</smiles><smiles>COc1cc(-c2cc(=O)c3c(O)cc(OC4OC(CO)[C@@H](O)C(O)[C@H]4O)cc3o2)ccc1O</smiles>

Figure 14. Chemical structures of compounds 55-76 isolated from mangrove species. 
<smiles></smiles>

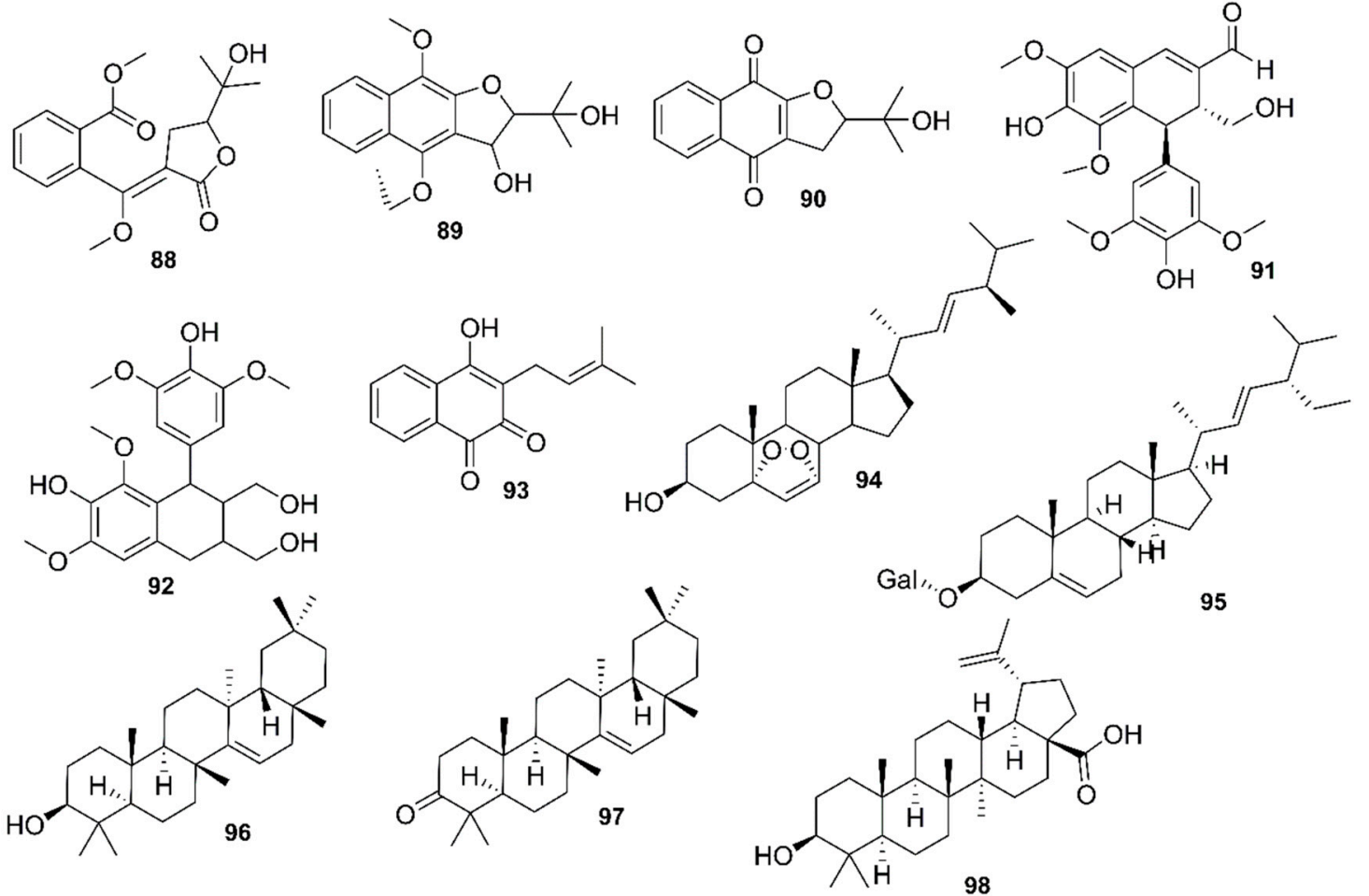

Figure 15. Chemical structures of compounds 77-98 isolated from mangrove species. 


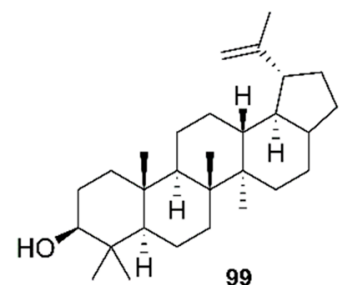

99

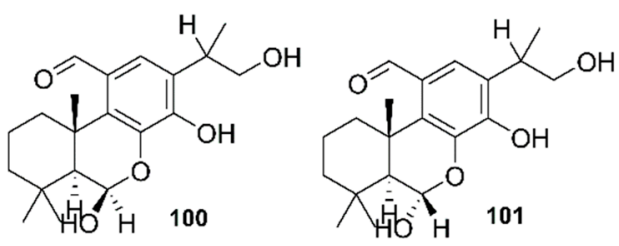<smiles>CC/C=C/C/C=C/C/C=C/CCCCCCCCC(=O)O</smiles><smiles>CCCCCCCCC=CCCCCCCC(=O)O</smiles>

103

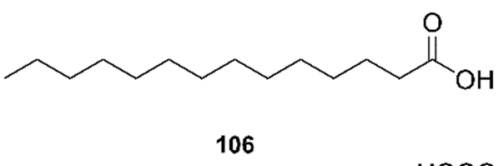<smiles>CC1(C)CC[C@H]2C(C(=O)O)=CO[C@@H](OC3O[C@H](CO)[C@@H](O)[C@H](O)[C@H]3OC(=O)/C=C/c3ccccc3)[C@]21C</smiles>
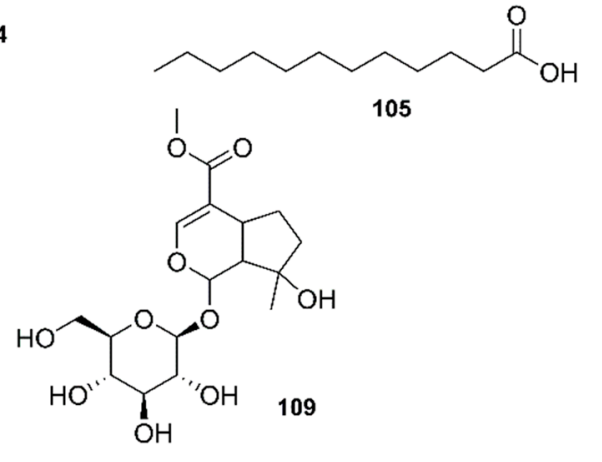

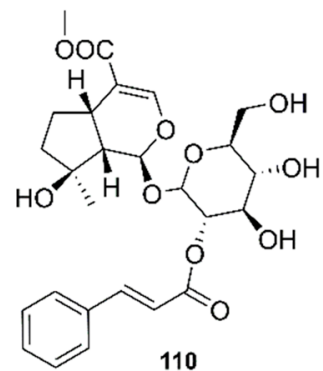<smiles>COC(=O)C1=CO[C@@H](OC2O[C@H](CO)[C@@H](O)[C@H](O)[C@H]2O)[C@H]2C(COC(=O)/C=C/C=C/c3ccccc3)=CC[C@H]12</smiles><smiles>COC1=COC(OC2OC(CO)[C@@H](O)[C@H](O)[C@H]2O)[C@]2(O)CC=C(COC(=O)/C=C/c3ccccc3)[C@H]12</smiles><smiles>O=C(/C=C/C=C/c1ccccc1)OC1CC(O)C(O)C(CO)O1</smiles>

Figure 16. Chemical structures of compounds 99-114 isolated from mangrove species. 

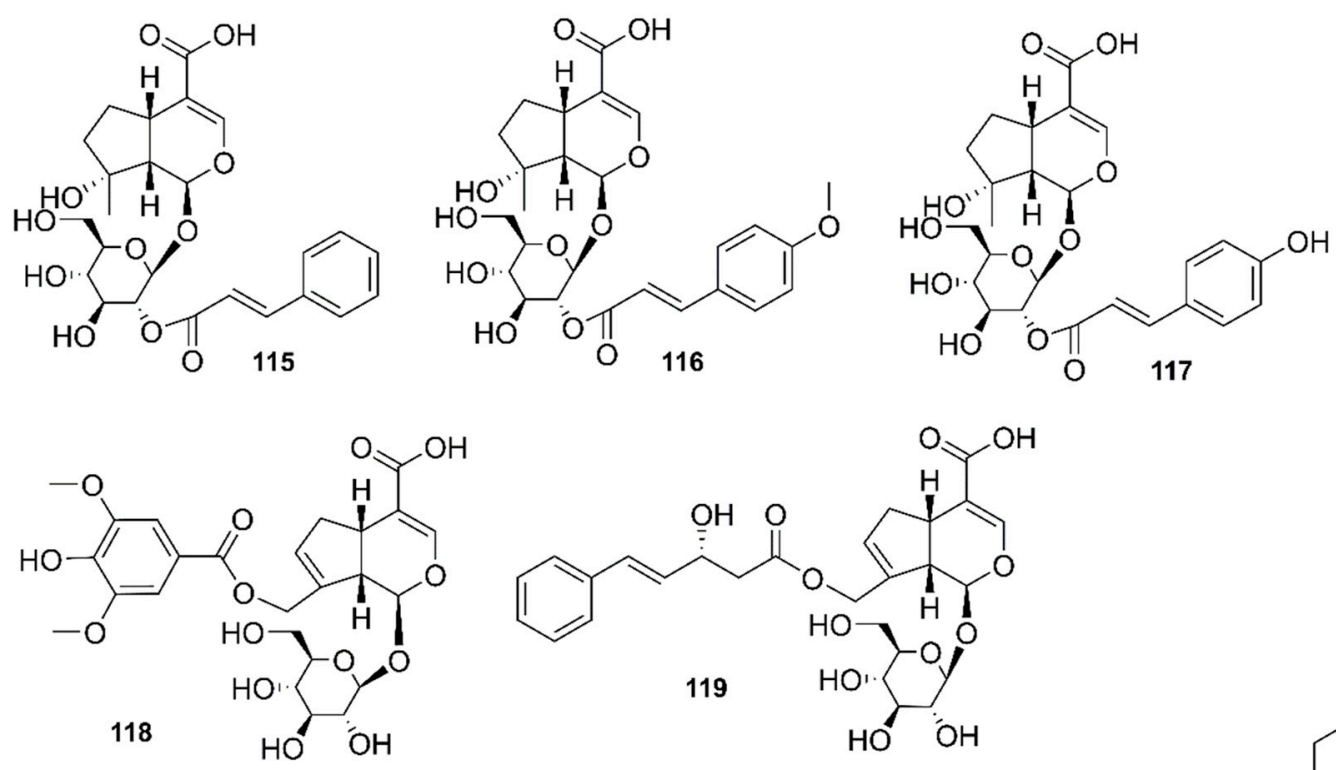

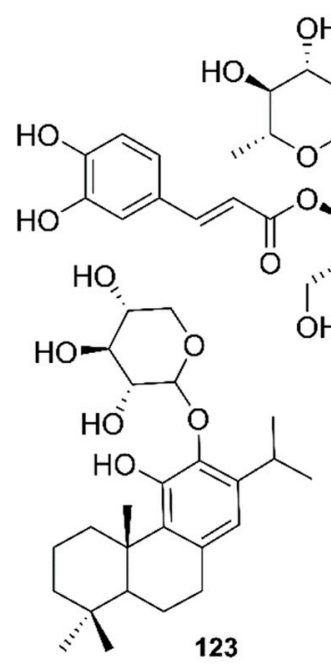<smiles>COc1cc(O)c2c(=O)cc(-c3ccc(O)c(OC)c3)oc2c1</smiles><smiles>COc1cc(C2c3c(cc(OC)c(O)c3OC)CC(CO)[C@H]2CO[C@H]2O[C@H](CO)[C@@H](O)[C@H](O)[C@H]2O)cc(OC)c1O</smiles><smiles>COC1O[C@H](COC(=O)/C=C/c2ccc(O)c(O)c2)[C@@H](O)[C@H](O[C@H]2O[C@H](C)[C@@H](O)[C@H](O)[C@H]2O)C1O</smiles><smiles>COCC/C=C/C(=O)O[C@H]1CC(O)[C@@H](OCCc2ccc(O)c(O)c2)O[C@H]1CO</smiles>
122

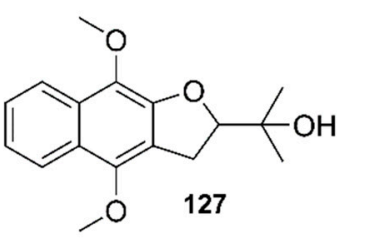<smiles>Oc1cc(O)c2c(c1)OC(c1ccc(O)c(O)c1)C(O)C2</smiles>

Figure 17. Chemical structures of compounds 115-128 isolated from mangrove species. 

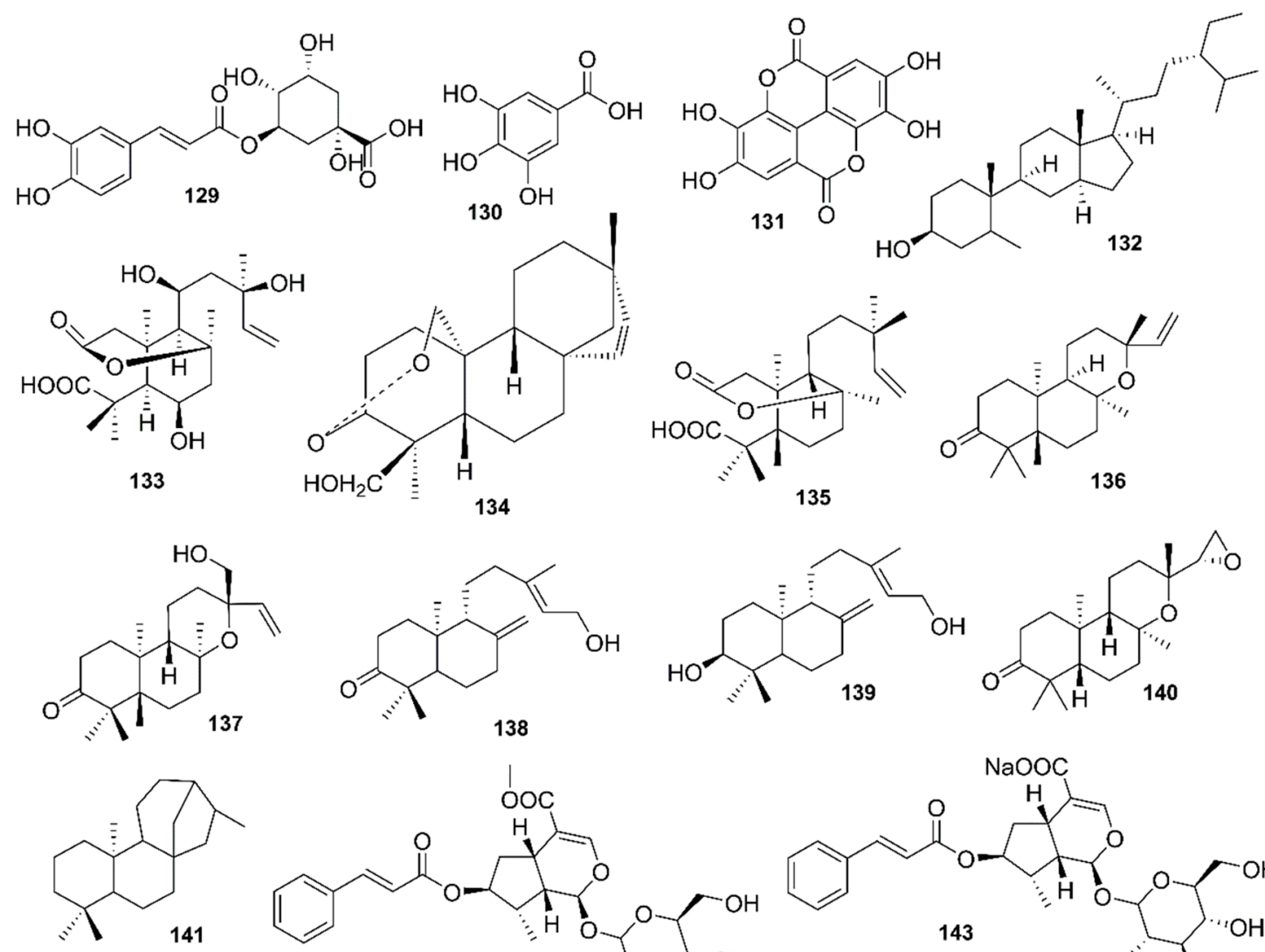

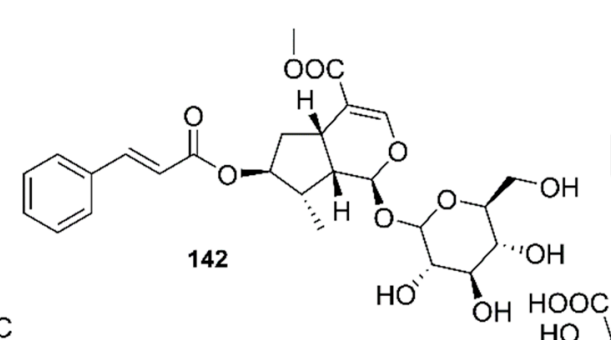

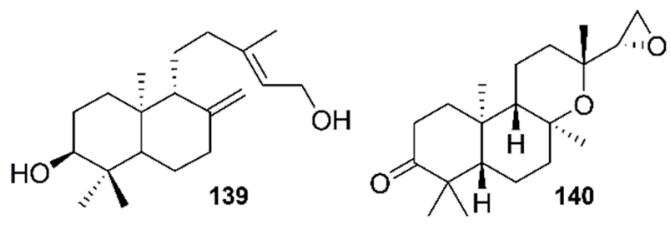<smiles>C[C@@H]1[C@@H](OC(=O)/C=C/c2ccccc2)C[C@]2(O)C(C(=O)O[Na])=CO[C@H](OC3O[C@H](CO)[C@@H](O)[C@H](O)[C@H]3O)[C@H]12</smiles><smiles>C[C@]1(OC(=O)/C=C/c2ccccc2)CC[C@H]2C(C(=O)O)=CO[C@H](OC3O[C@H](CO)[C@@H](O)[C@H](O)[C@H]3O)[C@H]21</smiles>
$\mathrm{HO}$

!<smiles>COCOC1OC(CO)[C@H](O)C(O)[C@H]1O</smiles>

Figure 18. Chemical structures of compounds 129-149 isolated from mangrove species. 


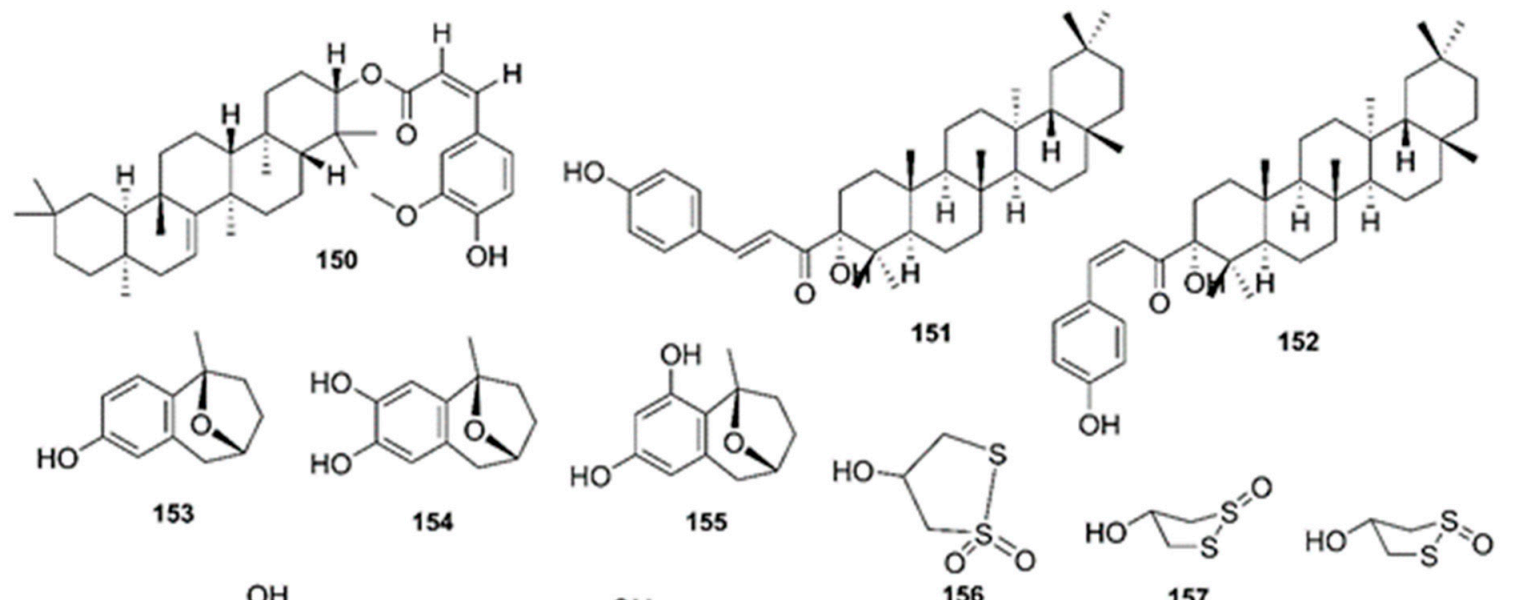

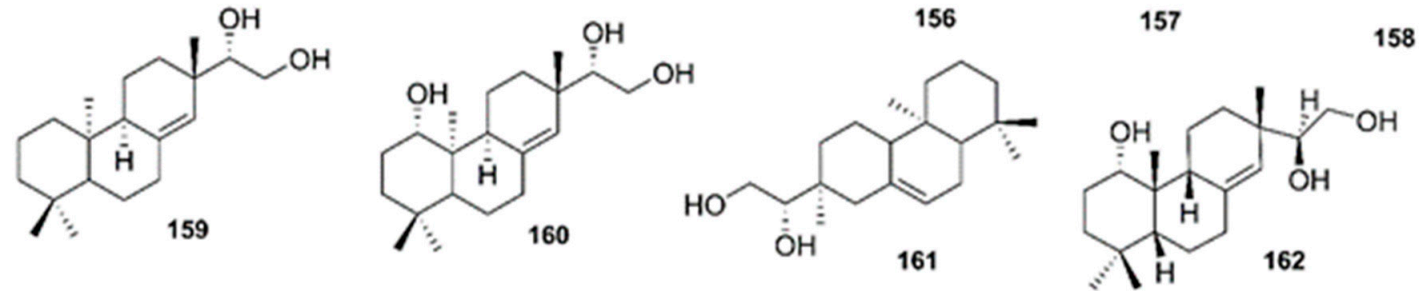<smiles>CC(O)CCC(O)Cc1cccc(O)c1</smiles>
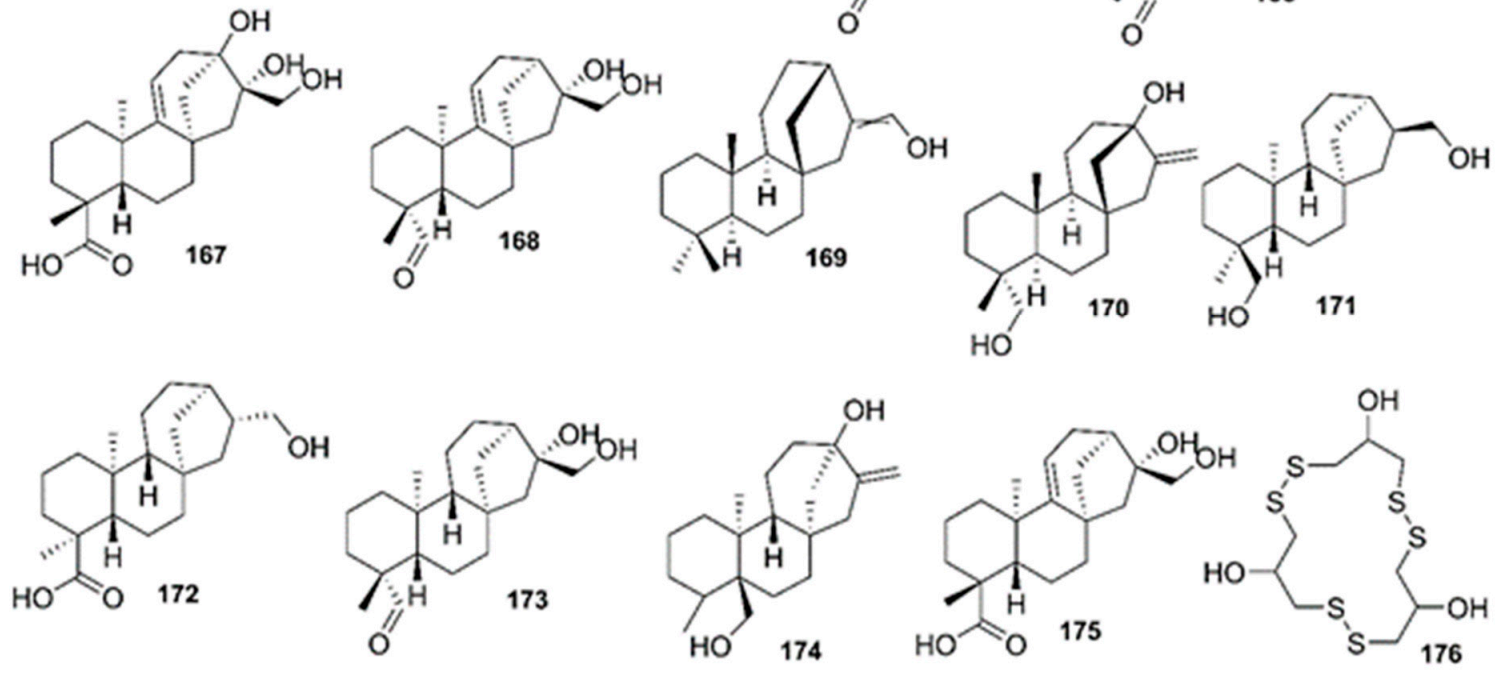

Figure 19. Chemical structures of compounds 150-176 isolated from mangrove species. 

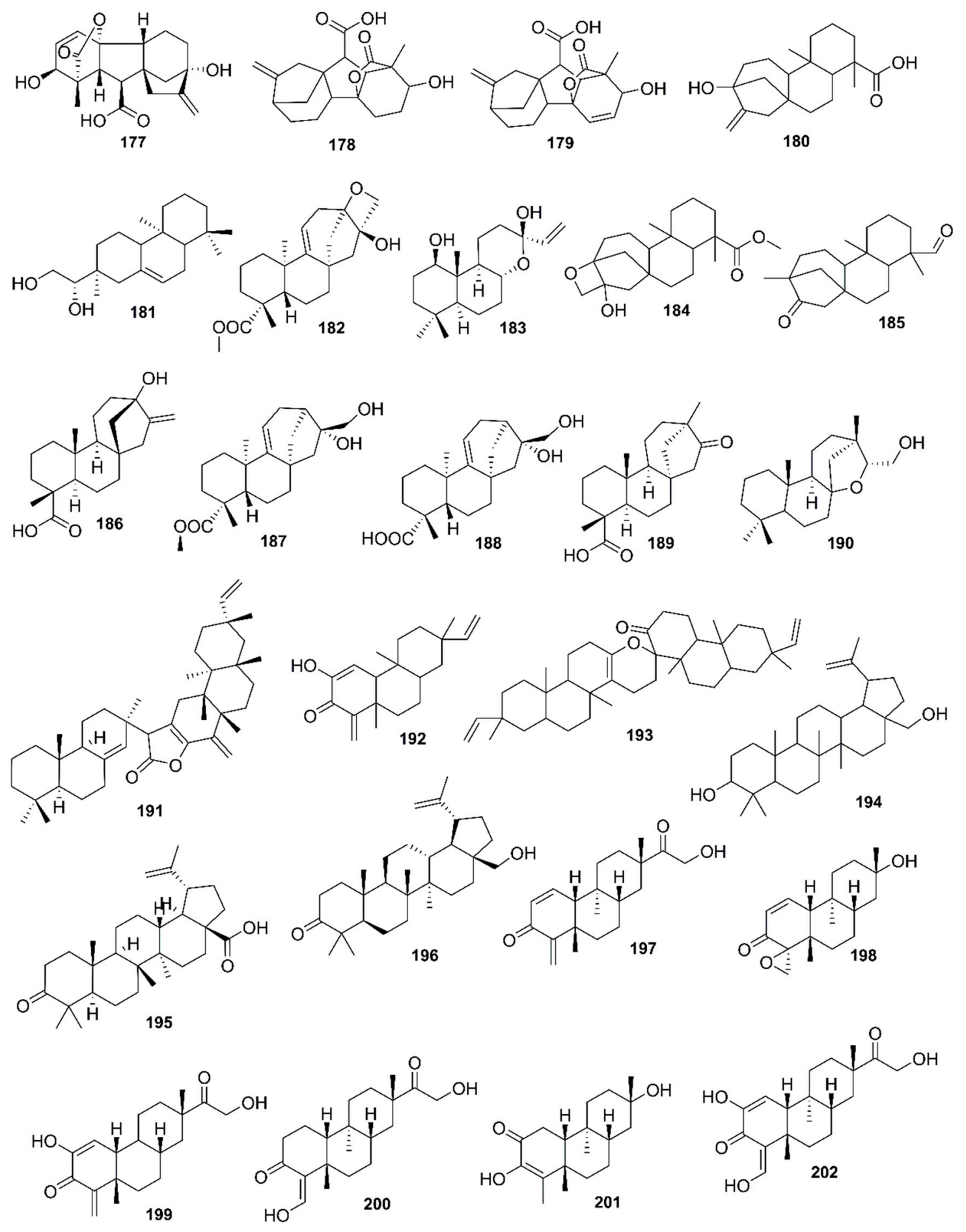

Figure 20. Chemical structures of compounds 177-202 isolated from mangrove species. 


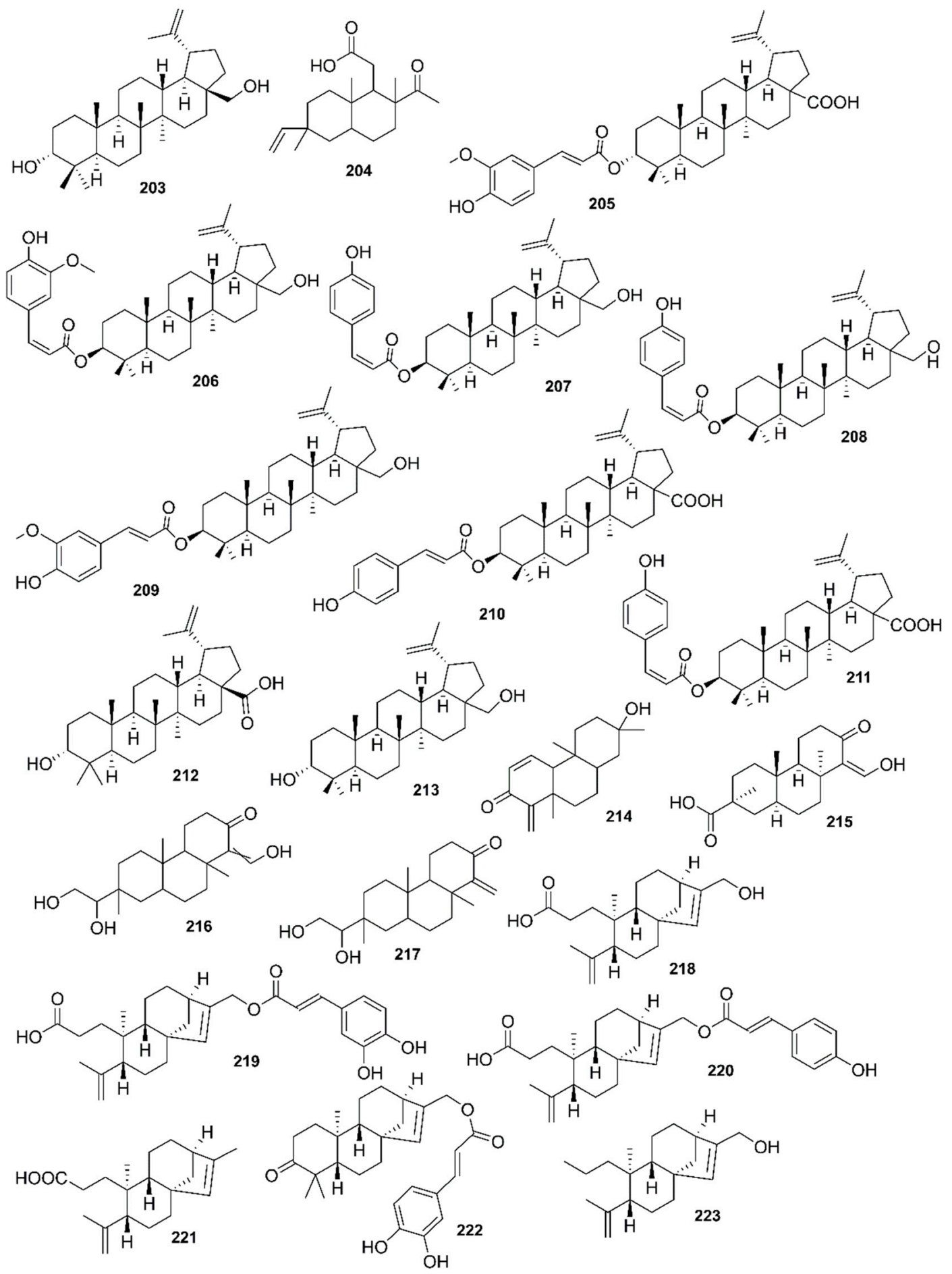

Figure 21. Chemical structures of compounds 203-223 isolated from mangrove speices. 


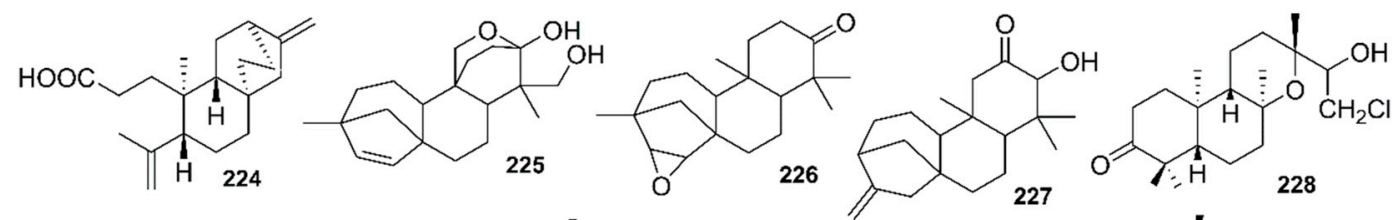

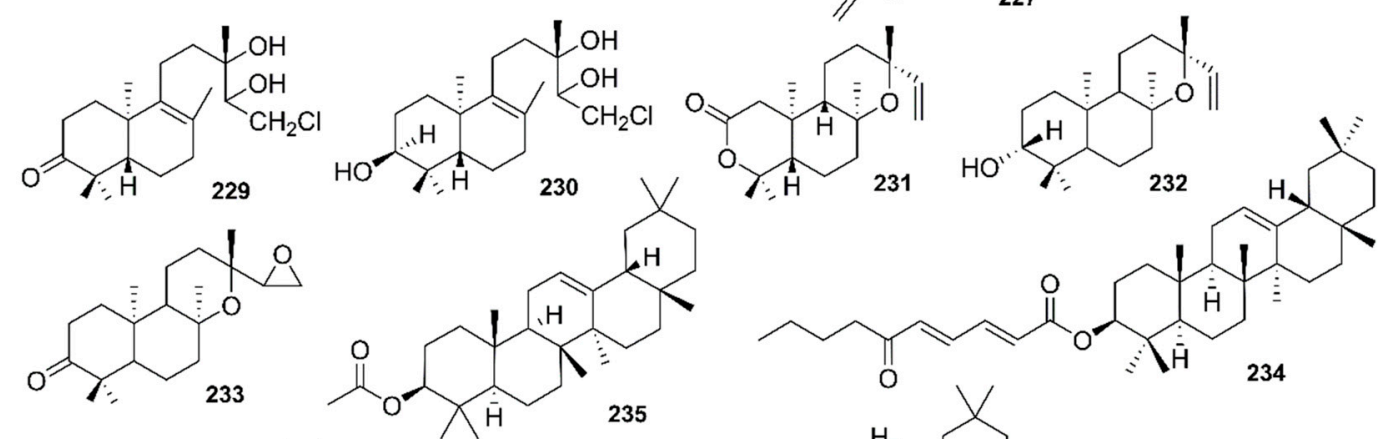

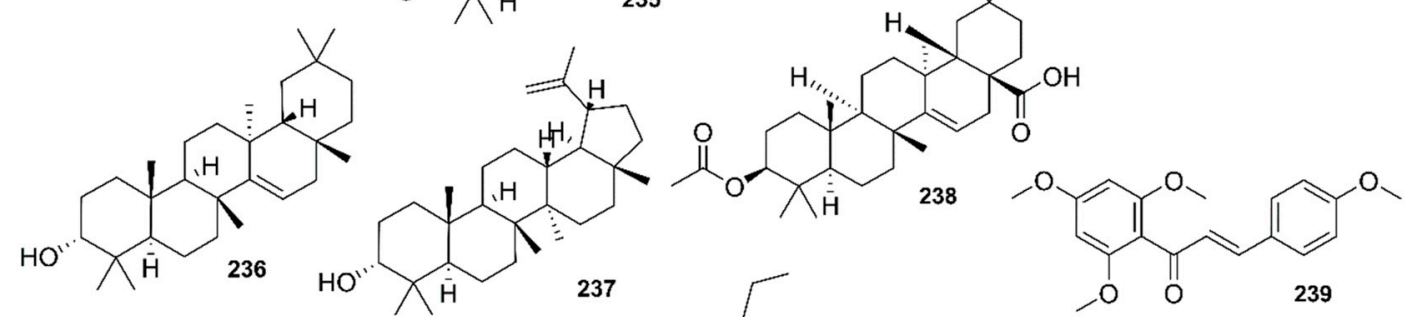<smiles>C=CO/C(C)=C\C(=C)O</smiles><smiles>COc1cc(OC(=O)OC2OC(Oc3cc(OC)c(OC)c(OC)c3)C(O)C(O)C2O)cc(OC)c1OC</smiles><smiles>CC1OC(Oc2c(-c3ccc(O)cc3)oc3cc(O)cc(O)c3c2=O)C(O)C(O)C(O)C1O</smiles><smiles>CCCCOCCOC(C)OCCCOC(C)OC1OC(C)[C@H](O)C(O)C1Oc1c(-c2ccc(O)c(O)c2)oc2cc(O)cc(O)c2c1=O</smiles>

Figure 22. Chemical structures of compounds 224-252 isolated from mangrove species. 
<smiles>Nc1ccc(C(=O)c2ccccc2)cc1</smiles><smiles>COc1cc(C2c3c(cc(OC)c(O)c3OC)CC(CO)[C@H]2CO[C@H]2OC[C@@H](O)[C@H](O)C2O)cc(OC)c1O</smiles>
258 I<smiles>C[C@@H]1OC(OC2Cc3c(O)cc(O)cc3O[C@H]2c2ccc(O)cc2)[C@H](O)[C@@H](O)[C@H]1O</smiles><smiles>COc1cc([C@H]2c3c(cc(OC)c(O)c3OC)CC(CO)[C@H]2CO[C@H]2OC(C)[C@@H](O)C(O)C2O)cc(OC)c1O</smiles>

257<smiles>COC1OC(CO[C@H]2O[C@H](C)C(O)[C@H](O)[C@H]2O)C(O)[C@@H](O)[C@H]1O</smiles><smiles>[CH]C(O)Oc1c(-c2ccc(O)c(O)c2)oc2cc(O)cc(O)c2c1=O</smiles>

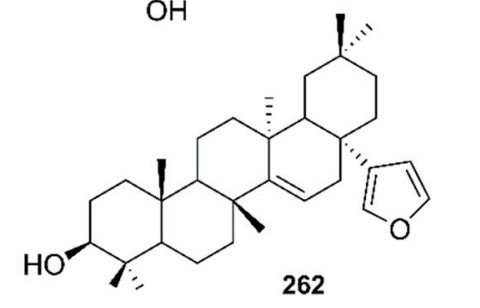

262

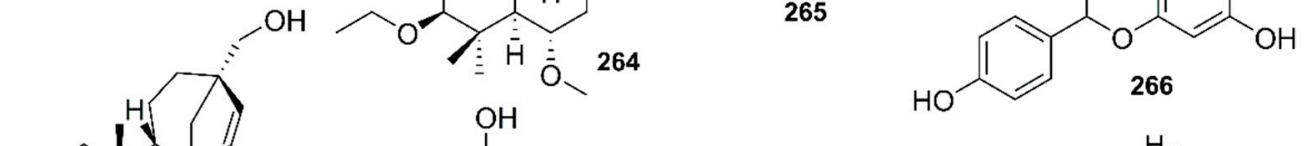

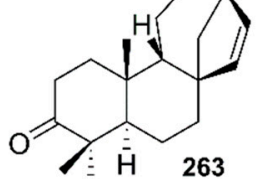<smiles>O=C1CC(c2ccc(O)c(O)c2)c2c(cc(O)c3c2OC(c2ccc(O)c(O)c2)CC3O)O1</smiles>

267<smiles>COc1cc(C2C(COC3OC[C@@H](O)C(O)[C@H]3O)Cc3cc(OC)c(O)c(OC)c3[C@@H]2CO)cc(OC)c1O</smiles><smiles>O=C1CC(c2ccc(O)c(O)c2)c2c(cc(O)c3c2OC(c2ccc(O)c(O)c2)C(O)C3)O1</smiles>

268<smiles>CC1OC(OC2Cc3c(O)cc(O)cc3O[C@@H]2c2ccc(O)c(O)c2)C(O)C(O)C1O</smiles><smiles>COc1cc(C2c3c(cc(OC)c(OC)c3OC)C[C@H]2CO)c(C[C@H]2OC(O)[C@@H](O)[C@@H](O)C2O)c(OC)c1O</smiles><smiles>C=CC(O)CCCC1C(=C)CCC2C(C)(C)CCCC12C</smiles><smiles>C=CC1(C)CCC2C(CCC3[C@@H](C)CCCC23C)O1</smiles>

Figure 23. Chemical structures of compounds 253-273 isolated from mangrove species. 


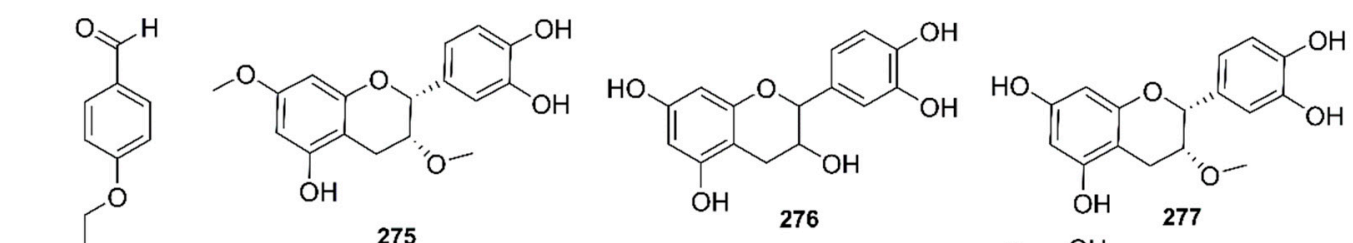<smiles>[Z20]CCC(C)CC</smiles>

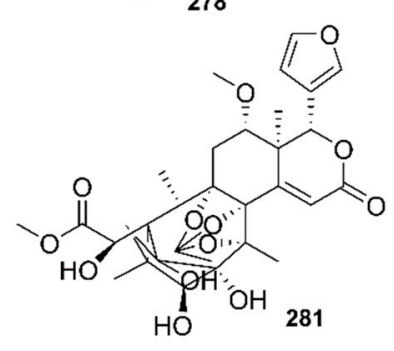<smiles></smiles><smiles>Cc1cc(O)c2c(c1)O[C@H](c1ccc(O)c(O)c1)[C@H](O)C2c1c(O)cc(C)c2c1O[C@H](c1ccc(O)c(O)c1)[C@H]2O</smiles>

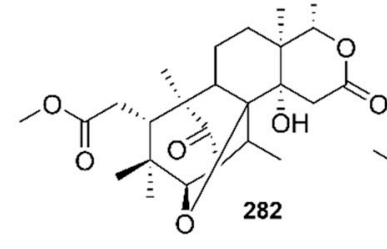

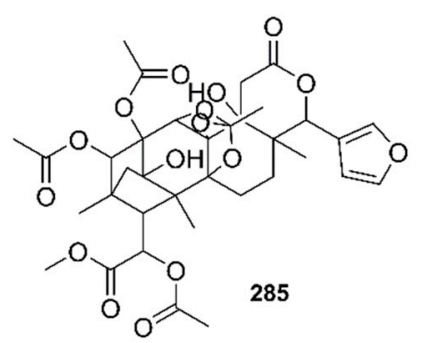

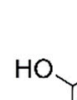<smiles>CC1Cc2ccc(O)cc2OC1c1ccc(O)c(O)c1</smiles>

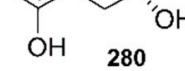

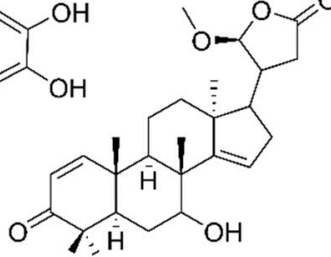

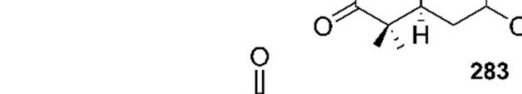


skin allergies, snake bites, diabetes, asthma, kidney stones, smallpox, and ulcer (Table 5). In South Thailand, this species is used to treat psoriasis [70].

A. ilicifolius is found to have many pharmacological activities; namely, the methanolic leaf extracts have good antioxidant, anti-inflammatory activities [66,105]. Acetone leaf extracts showed good antimicrobial activities. A wide array of phytochemical constituents is found to be present in different parts of the plants namely the leaves, barks, roots, and fruits. Results from GC/MS confirmed the presence of alkaloids (acanthicifoline (3), benzoxazin-3-one (6)), flavonoids, steroids (cholesterol (51), $\beta$-sitosterol (54)), glycosides, saponins, tannins, and terpenoids [42,155,156] (Table 8). Ribose derivative isolated from this mangrove species known as 2-benzoxazoline exhibited antiviral and antitumor activities [150]. Recently, a new sugar ester was derived from the roots of A. ilicifolius known as 1,2-di-(syringoyl)- $\beta$-D-glucopyranose [157]. The structure of the new compound was clarified by extensive spectroscopic methods such as NMR and HRESI-MS.

\subsection{Aegialitis rotundifolia}

A. rotundifolia is a small tree originating from the Plumbaginaceae family with a height of 2-3 m. It has broad and ovate leaves, $5-8.8 \mathrm{~cm}$ long and $4.5-8.5 \mathrm{~cm}$ wide $[44,45]$. The species is mainly grown in Bangladesh and is used to cure inflammatory and painful arthritis [72,73]. The leaf infusion is used as an anti-ache agent. A. rotundifolia showed moderate inflammatory and anti-pyretic activities with aqueous leaf extracts [73]. Moreover, with recent literature, methanolic leaf extracts showed thrombolytic and membrane stabilizing activities. Additionally, the extracts did not show any antibacterial activity as the test sample was resistant against both gram-positive and gram-negative bacteria [45]. Ghosh et al. [160] identified compounds comprising of alkaloids, carbohydrates, tannins, phenolic compounds, sterols, triterpenoids, saponins, and flavonoids from the ethanolic leaf extracts.

\subsection{Aegiceras corniculatum}

A. corniculatum originates from the Primulaceae family with a height of up to $7 \mathrm{~m}$. It has alternate and obovate leaves, $3-10 \mathrm{~cm}$ long and $1.5-5 \mathrm{~cm}$ wide. Its fruit is green to pink in color and has a curved-cylindrical shape [43]. Folk medicinal practitioners from the Sindh region in Pakistan use the stem to treat rheumatism, painful arthritis, and inflammatory diseases [74,75]. A. corniculatum showed many potential pharmacological activities (Table 6). For instance, the in vivo antinociceptive activity was investigated by Roome et al. $[74,75]$ using acetic-acid induced writhing in mice. The ethyl acetate stem extracts at $50 \mathrm{mg} / \mathrm{kg}$ showed an inhibition of $53 \pm 3.0 \%$ while the hexane stem extract at the same concentration has an inhibition of $28 \pm 2.5 \%$. Janmanchi et al. (2017) conducted an antibacterial study using REMA assay. It was found that the crude leaf extract was active against Bacillus subtilis and Escherichia coli. With the same extract, the antioxidant study was conducted using DPPH assay, and the $\mathrm{IC}_{50}$ value was $1.79 \pm 0.0002 \mathrm{mg} / \mathrm{mL}$. However, Roome et al. [74] mentioned that $A$. corniculatum lacked pharmacological evaluation concerning its analgesic effects. Using standard phytochemical tests, analysis of the bark, stem, and leaf extracts showed the presence of alkaloids, amino acids, benzoquinones, tannins, coumarins, flavonoids, saponins, and glycosides, among others (Table 8).

\subsection{Acrostichum aureum}

This species is found in Kerala, India. The local people used the whole plant as a worm remedy and as an astringent for hemorrhage [76]. Thomas [76] investigated the antibacterial activity of methanol, acetone, petroleum ether, and water leaf extracts against Escherichia coli, Serratia marcesens, Pseudomonas aeruginosa, and Staphylococcus aureus. The petroleum ether and water leaf extracts were inactive against Escherichia coli and Serratia marcesens while acetone leaf extract was active against all the tested microorganisms. In the methanol, acetone, petroleum ether, and water leaf extracts, alkaloids were reported absent whereas flavonoids and phenols were found present [76]. 


\subsection{Avicennia}

A. marina is a medium length mangrove tree from the Acanthaceae family with a height of $14 \mathrm{~m}$ with a specialized root structure known as pneumatophores. Its bark is smooth with thin, stiff, and brittle flakes in the surface and is generally light grey. The leaves are thick and glossy and 5-8 cm long. The species produces green and oval-shaped fruits [7,46]. A. marina is widely distributed in Australia, South-East Asia, Madagascar, Mozambique, and along the coastline of Africa [7]. This species traditionally used to manage smallpox, skin diseases, ulcers, and throat pains [65] and it has many pharmacological properties such as antimicrobial, anti-inflammatory, antiviral, antimutagenic, anticancer, and antioxidant (Table 6). Ramanathan [114] investigated the antimicrobial activity of the crude leaf extract using disc diffusion assay against S. aureus, Klebsiella aerogenes, P.s aeruginosa, Bacillus subtilis, E.a coli, Enterobacter aerogenes, Proteus sp, Salmonella parathyphi, and Citrobacter sp. The extracts showed activity against all the tested microorganisms. Shafie et al. (2013) conducted the anti-inflammatory activity on the rat model, and it was observed that the inflammatory markers were reduced, and the joint lesions were also improved. The ethanolic leaf extracts were active against HIV (Human immunodeficiency virus), SFV (Semliki forest virus), EMVC (Encephalmyocarditis virus), and HBV (Hepatitis B virus) [98]. The phytochemical screening of the methanolic, ethanolic, ethyl acetate, ethyl ether, and water extracts indicated the presence of a wide array of constituents viz; alkaloids, 31 glycosides, phenols, 5 terpenoids, saponins, 14 flavonoids, 23 tannins, 19 naphthalene derivatives, 6 fatty acids, and 7 steroids and amino acids [7] (Table 8).

A. germinans is the tallest mangrove tree compared to the other Avicennia species such as A. integra, A. bicolor, A. marina, A. officinalis, and A. schaeurina. This species comes from the Acanthaceae family. It is 30 to $50 \mathrm{~m}$ tall with rough and irregular scales on the bark. These plants have opposite and elliptical leaves which are 3-15 cm long. It produces dark-green, flat propagules with velvety pericarp which are 2-3 cm in diameter [7]. The bark, leaf, and flower of $A$. germinans are used traditionally to treat malaria, haemorrhoids, rheumatism, swellings, throat pains, and hemorrhage [7,77]. In the Bahamas, A. germinans is traditionally used to restore vitality and to manage rheumatism while in Colombia, gargling the bark decoction helps to cure cancer of larynx and ulcers of the throat [77]. The methanol extract of A. germinans exhibited significant antibacterial activity against Escherichia coli, Klebsiella sp, Proteus sp, Staphylococcus aureus, Pseudomonas sp., and Salmonella sp. Fennell et al., (2004) reported that the antibacterial properties are due to the presence of tannins, alkaloids, flavonoids, terpenoids, or essential oils. The compounds identified from $A$. germinans originate from the phytochemical class of glycosides namely 2'-cinnamoyl-mussaenosidic acid (108), 2' -caffeoyl-mussaenosidic acid, and 2'-CoU mamaheswarraoroyl-mussaenosidic acid [7,161,162].

A. integra is the smallest mangrove tree with a height of $2-7 \mathrm{~m}$ in the Avicennia genus. It has pneumatophore roots system with smooth bark, brown to reddish. The leaves are opposite, simple, and elliptical with shiny surfaces of length $5-14 \mathrm{~cm}$. The plant produces pale green fruits, $21-23 \mathrm{~mm}$ long and 12-15 mm wide. This species also blooms to produce golden yellow or orange zygomorphic flowers. The plant can be found along the coastline of Australia [7].

A. bicolour originates from the Acanthaceae family and is $8-20 \mathrm{~m}$ tall. It is widely distributed in Colombia, Costa Rica, El Salvador, Guatemala, Honduras, Mexico, Panama, and Nicaragua [7].

A. schauerina species comes from the same family of Acanthaceae as the other Avicennia mangrove trees. The produced fruits are pale sap green with a purple tinge and are flatter compared to A. germinans. A. schauerina produced flowers which are larger than the flowers produced by A. bicolor [7].

A. officinalis is $30 \mathrm{~m}$ tall with pneumatophore roots system, smooth bark which is dirty green to dark grey, and is slightly fissured but does not flake compared to A. germinans [47]. The leaves are shiny, green in color with round apex, $10 \mathrm{~cm}$ long, and $5 \mathrm{~cm}$ wide. The tree has pneumatophores similar to the other Avicennia species. The flower of A. officinalis is the largest of all the species in its genus and is orange-yellow to lemon-yellow. This species produced a heart-shaped propagule, green or brown $[7,47]$. A. officinalis is an evergreen mangrove tree distributed throughout India, Bangladesh, Indonesia, Brunei, Myanmar, Vietnam, and Southern Papua New Guinea (Hossain et al., 2016). In Bangladesh, the species 
is known as 'DholaBaen'. Locally, it is used as a treatment for boils and tumors (Hossain et al., 2016) and the unripe seeds are poulticed onto the sores of smallpox, boils, and abscesses [156]. Additionally, the bark can be used to heal scabies (Hossain et al., 2016). A decoction of the plant mixed with sugar candy and cumin is used against dyspepsia. The local people traditionally use the resin produced by the plant as a contraceptive without side effects [80]. A. officinalis is largely studied for its pharmacological activities. For instance, the ethyl acetate leaf extract is analyzed for its antimicrobial activity against E. coli, Streptococcus mutans, S. aureus, Aspergillus flavus, and Trichophyton rubrum. The extract showed activity against E. coli, S. mutans, and S. aureus but found inactive for A. flavus and T. rubrum. Anti-ulcer activity was investigated on the ethanolic extract using indomethacin-induced gastric ulcer assay and it was observed that the gastric ulcers decreased when the amount of glutathione is reduced in the gastric mucosa [115]. Hossain et al. (2012) investigated the diuretic and neuropharmacological properties of the methanolic leaf extracts. The Lipschitz diuretic model was used to test the diuretic activity of the sample. For the dosage of 200 and $400 \mathrm{mg} / \mathrm{kg}$, the volume of urine excreted was $3.06 \pm 0.18 \mathrm{~mL}$ and $3.89 \pm 0.13 \mathrm{~mL}$, respectively. It is reported that the amount of $\mathrm{Na}^{+}$ion excreted by the methanolic extract is higher compared to the excretion of $\mathrm{K}^{+}$ion and as a result the plant is classified as a good diuretic which causes less hyperkalaemic side effects. In GC/MS analysis, the methanolic, ethanolic crude leaf extract showed the presence of alkaloids, terpenoids, glycosides, tannins, steroids, flavonoids, naphthalene derivatives, reducing sugar, sterols, fatty acids, gums, wax esters, and amino acids, among others. Thatoi et al. [7] identified 17 compounds from the terpenoid class of constituents as taraxerol (96), taraxerone (97), betulinic acid (98), betulin (99), betulinaldehyde (125), $\beta$-amyrin (47), rhizophorin-A (133), rhizophorin-B (134), ent-13S-2,3-seco-14-labden-2,8-olide-3-oic acid (135), ribenone (136), ent-16-hydroxy-3-oxo-13-epi-manoyl oxide (137), ent-15-hydroxy-labda-8,13E-dien-3-one (138), ent-3 $\alpha, 15$-dihydroxylabda-8,13E-diene (139), excoecarin A (140), ent-beyerane (141), rhizophorin-B (134); nine glycosides compounds as 7-O-trans cinnamoyl-4-epilogenin (142), geniposidic acid (107), 2'-cinnamoyl-mussaenosidic acid (108), 10-O-5-phenyl-2,4-pentadienoyl-geniposide (111), 7-O-cinnamoyl-8-epiloganic acid sodium salt (143), 8-O-cinnamoylmussaenosidic acid (144), officinosidic acid (145), loganin C, (146) iridoid glucoside; five steroids compounds as $\beta$-sitosterol (54), stigmasterol (53), cholesterol (51), campesterol (52), stigmast-7-en-3-ol (55); four tannins compounds as catechin (128), chlorogenic acid (129), gallic acid (130), ellagic acid (131); one naphthalene derivative as avicenol C (127); and one flavonoid compound as velutin (126) (Table 8).

\subsection{Bruguiera}

This species has not received enough scientific attention concerning its morphological characteristics, traditional uses, and pharmacological properties. However, Revathi et al. [88] and Bandaranayake [65] reported that the stem and bark of the plant consist of sulfur-containing alkaloids.

B. cylindrica coming from the Rhizophoraceae family, is $20 \mathrm{~cm}$ tall with pneumatophore roots and has smooth bark, grey with corky raised patches containing lenticels. The leaves are glossy in appearance and elliptical in shape with pointed apex. The plant produces fruits of $15 \mathrm{~cm}$ long and has a curved-cylinder shape. This species blooms greenish-white flowers in clusters of two to five [48]. The bark of B. cylindrica is traditionally used to treat hemorrhage and ulcers by the local people of India [63]. The $\mathrm{IC}_{50}$ values for the methanolic leaf and stem extracts are 175 and $162.5 \mu \mathrm{g} / \mathrm{mL}$, respectively [120]. Laphookhieo et al. [163] conducted phytochemical screening on the fruit of B. cylindrica and the pentacyclic triterpenoids esters identified are E-feruloyltaraxerol (147), 3 $\alpha$-Z-feruloyltaraxerol (148), 3 $\beta$-E-feruloyltaraxerol (149), 3 $\beta$-Z-feruloyltaraxerol (150), $3 \alpha$-E-coumaroyltaraxerol (151), and $3 \alpha$-Z-coumaroyltaraxenol (152). Gawali and Jadhav [120] reported the presence of tannins, saponins, alkaloids, triterpenoids, anthraquinone, and flavonoids in the leaves of the plant.

The bark of this mangrove tree is used to manage diabetes [61]. Bunyapraphatsara et al. [123] performed antioxidant tests on the ethyl acetate leaf extract using three different methods namely DPPH, lipid peroxidation inhibition, and quinone reductase induction activity. Using the DPPH assay, the resulting $E_{50}$ values of the young pods and the leaves are 5 and $105 \mu \mathrm{g} / \mathrm{mL}$, respectively. With lipid 
peroxidation inhibition assay, the $\mathrm{IC}_{50}$ values of the young pods and leaves are 0.375 and $42.6 \mu \mathrm{g} / \mathrm{mL}$, respectively. Also, while using the third method, which is quinone reductase induction assay, the $\mathrm{IC}_{50}$ values recorded were $>20 \mu \mathrm{g} / \mathrm{mL}$ for both young pods and leaves samples. Comparing the $\mathrm{IC}_{50}$ values from the two methods (lipid peroxidation inhibition and quinone reductase induction), it can be said that the samples showed better inhibition with the quinine reductase induction assay. Arora et al. [101] reported the presence of phenolic compounds in the bark of B. parviflora. Revathi et al. [88] reported the presence of tannins and triterpenes in the bark and leaves of the plant.

B. sexangula is scarcely distributed on the north shore and sides of Oahu, Hawaii [186]. Revathi et al. [88] reported the presence of phenolics, steroids, alkaloids, and tannins in the bark of this species. Alkaloid (1,2-dithiolane) of this plant exhibited antitumor activity against Sarcoma 180 and Lewis [150].

B. gymnorhiza is a common mangrove tree reaching a height of up to $15 \mathrm{~m}$ and originates from the Rhizophoraceae family. Its bark is smooth and grey-brown in color. It has smooth and glossy leaves with pointed apex, 9.5-20 cm long and 3-7 cm wide. The propagules are green in color and have a cigar-shape which is $5-12 \mathrm{~cm}$ long and 1-2 cm wide. The flowers of the plant have pale yellow-green to pinkish orange sepals [187]. B. gymnorhiza is a well-known mangrove tree. This species is distributed in the wild forest of India (Sunderbans), throughout Malaysia, China, Indonesia, Comoros, and Mauritius. In India, the bark and root decoction is used to treat diabetes, fever, and diarrhea [81,82]. In Malaysia, the local people used its stem as a remedy for viral fever [83]. In the Guangxi Province of China, the leaves and fruits are traditionally used to cure burns, intestinal worms, liver disorders, and diarrhea [33,54]. The folk medicine practitioners in Indonesia uses the fruits to treat eye disease, malaria, and shingles, which is a viral infection that can occur anywhere on the body [85]. In Comoros and Mauritius Islands, a decoction is prepared by boiling root ( $15 \mathrm{~cm}$ length) of B.gymnorhiza and five to seven leaves of $P$. borbonense in two cups of water. The decoction is taken to manage diabetes, hypertension, and hemorrhage [13]. The leaves, roots, and barks of this species have been reported to possess many medicinal properties (Table 5). The methanolic leaf extract has been studied for its antinociceptive activity using acetic acid-induced writhing in mice. At dosage 250 and $500 \mathrm{mg} / \mathrm{kg}$, the $\%$ writhing inhibitions were $46 \%$ and $59 \%$, respectively. The extract showed significant inhibition compared to the standard drug diclofenac sodium and confirmed the antinociceptive activity [81]. Barik et al. [121] investigated the anti-inflammatory activity on the crude leaf extract using COX (cyclooxygenase) inhibition assay. The \%inhibitions at dosage 10 and $10 \mu \mathrm{g} / \mathrm{mL}$ were $9.7 \pm 7.2 \%$ and $65.1 \pm 5.8 \%$, respectively. The ethanolic root extract was reported non-toxic with no significant change in behavior or neurological response up to $400 \mathrm{mg} / \mathrm{kg}$ body weight [82]. Methanolic leaf extract was found active against Escherichia coli $(22 \mathrm{~mm})$, while the hexane bark extract showed a broader spectrum of antimicrobial activity against K. pneumonia (23 mm), S. typhi (22mm), Staphylococcus aureus (19 $\mathrm{mm})$, and Shigella flexneri $(22 \mathrm{~mm})$, respectively [62]. The plant was also reported to exhibit antioxidant, antihyperglycemic, anti-diarrheal, and hepatoprotective activities [64,81-83,105] (Table 7). Phytochemicals present in the leaves, stems, flowers, roots, and fruits include flavonoids, saponins, reducing sugars, tannins, gums, dammarane triterpenes, aromatic compounds, sterols, diterpenoids, anthocyanins, and catechins, among others (Table 8). Rahman et al. [164] identified the compounds from the dammarane triterpenes class as Bruguierol A-C (153-155), 4-hydroxy-dithiosulfonate, bruguiesulfurol (156), 4-hydroxydithiolane 1-oxides, brugierol (157), and isobrugierol (158).

\subsection{Ceriops}

The whole plant of $C$. roxburghiana species is traditionally used to treat diabetes and ulcers. The tree originates from the Rhizophoraceae family. Phytochemical screening of the whole plant revealed the presence of gibberellins and procyanidins [88].

C. decandra is native to India in the Tamil Nadu region. The local people use its bark, flowers, and leaves to treat hepatitis and ulcers [87]. This species showed various pharmacological properties namely antioxidant, anti-inflammatory, anti-microbial, and anti-HIV activities (Table 7). Premanathanet al. (1996) investigated the anti-HIV activity on the leaf extract using MTT (3-(4,5-dimethylthiazol-2-yl)-2, 
5-diphenyltetrazolium bromide) assay. The $\mathrm{CC}_{50}$ and $\mathrm{EC}_{50}$ values of the leaf extract were $216.54 \pm$ 14.21 and $13.38 \pm 3.15 \mu \mathrm{g} / \mathrm{mL}$, respectively. The leaf extract showed an SI value of 16.18 . The higher the SI value, the lower the toxicity towards the host cells and, thus, the higher its effects against the virus. Comparing the SI values of the leaf extract of $C$. decandra with the values of $R$. apiculata (9.19), R. mucronata (1.62), R. larmarckii (2.38), respectively, it can be said that the leaf extract of $C$. decandra has higher anti-HIV activity. The ethyl acetate leaf extract was active against the following bacterial pathogens: E. coli, Agrobacterium tumefaciens, S.s mutans, and S.s aureus, while the same extract was found inactive against A.s flavus and T. rubrum [106]. The ethanolic leaf extract showed antioxidant and anti-inflammatory activities [125]. Revathi et al. [88] reported the presence of polyphenols, tannins, and triterpenes in the bark, leaf, and fruit extracts. Anjaneyulu and Rao [167] identified the following compounds: Ceriopsins F-G (184, 185), ent-13-hydroxy-16-kauren-19-oic acid (186), methyl ent-16ß,17-dihydroxy-9(11)-kauren-19-oat (187), ent-16ß,17-dihydroxy-9(11)-kauren-19-oic acid (188), ent-16-oxobeyeran-19-oic acid (189), and 8,15R-epoxypimaran-16-ol (190) from the ethyl acetate root extract of the plant. Other phytochemicals isolated from the hexane, acetone, chloroform, and methanol leaf extracts include alkaloids, flavonoids, steroids, phenols, saponins, terpenoids, carbohydrates, reducing sugars, and cardiac glycosides, among others (Table 8) [168,169].

C. tagal comes from the Rhizophoraceae family and grows to a height of $25 \mathrm{~m}$. It has a buttress root system, smooth barks, and is silvery-grey to orangish-brown with lenticels on its surface. The leaves are obovate and yellowish-green on the bottom surface, and they are $6 \mathrm{~cm}$ long and $3 \mathrm{~cm}$ wide. The propagule is ovoid in shape, brown in color, and is generally $3 \mathrm{~cm}$ long [57]. The bark of this plant is traditionally used to treat hemorrhage [61]. Chen et al. [170] identified six compounds from

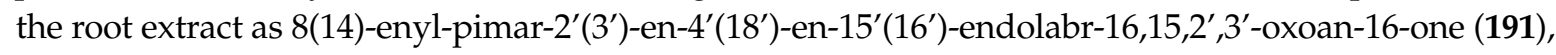
tagalsin C (192), tagalsin I (193), lup-20(29)-ene-3 $\beta$,28-diol (194), 3-oxolup-20(29)-en-28-oic acid (195), and 28-hydroxylup-20(29)-en-3-one (196), while Wang et al. [29] identified 14 compounds from the ethanolic root extract as $3 \alpha-O$-trans-feruloylbetulinic acid, $3 \alpha$-O-trans-coumaroylbetulinic acid (205),

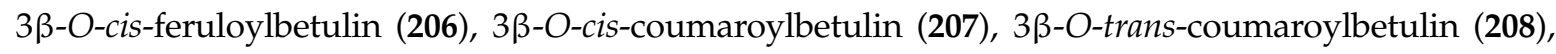

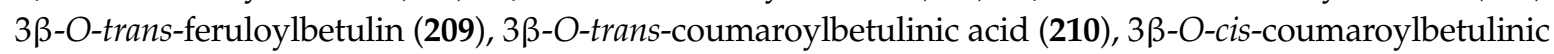
acid (211), lupeol (48), 3-epi-betulinic acid (212), betulin (105), 3-epi-betulin (213), and 28-hydroxylup-20(29)-en-3-one (196). Hu et al. [173] also isolated phytochemicals such as Dolabranes (Tagalsins P (201), Q (214), R (215), S (200), T (216), U (217)), pimarane, and abietane (Table 8).

\subsection{Excoecaria agallocha}

This species is also known as the blind-your-eye mangrove plant. This is because the latex produced by the bark is poisonous and can cause temporary blindness. E. agallocha comes from the Euphorbiaceae family and is $15 \mathrm{~m}$ tall. Its root system is described as elbow shaped pegs. The leaves are alternate and elliptical, with an acuminate apex and narrow base. They are $3-8 \mathrm{~cm}$ long and $1.5-3 \mathrm{~cm}$ wide [50]. This tree is known to produce latex which has therapeutic effects. The plant is traditionally used to treat rheumatism, epilepsy, leprosy, ulcers, and paralysis. This species is widely distributed from India, Africa, to northwest Australia. The local people of India, New Caledonia, and Malaysia traditionally used the latex and leaves to cure dart and fish poisoning. In Pakistan, besides using it for ulcers, paralysis, rheumatism, and leprosy, the latex is used as an abortifacient [174]. In Tamil Nadu, the latex is also used to alleviate a painful toothache [58]. This mangrove species possesses many pharmacological activities namely antioxidant, anti-inflammatory, analgesic, anticancer, anti-filarial, and antimicrobial activities (Table 7). Mondal et al. [50] investigated the anti-inflammatory activity on the stem extract using two different methods namely carrageenan-induced paw edema test and pellet-induced granuloma test. The ethanol and water (3:1) extract of the plant showed a significant inhibition of $62.29 \%$ in carrageenan-induced paw edema model while the pellet-induced granuloma test showed an inhibition of $57.03 \%$ with the stem extract. Analgesic activity was tested using acetic acid-induced writhing test in mice. At dosage $500 \mathrm{mg} / \mathrm{kg}$, the ethanol and water (3:1) bark extract showed the highest activity with a reduction of $53.87 \%$. The antimicrobial properties of E. agallocha 
were tested by Bakshi and Chaudhuri [106] using disc-diffusion assay and the results showed the extract was active against $E$. coli, $A$. tumefaciens, $S$. mutans, and $S$. aureus but inactive against $A$. flavus and T. rubrum. A study by Mondal et al. [50] revealed a wide array of phytoconstituents isolated from E. agallocha (Table 8). The main constituents identified were flavonoids, terpenoids, diterpenes, alkaloids, and tannins [50,88,174-176].

\subsection{Heritiera}

$H$. fomes is a tall mangrove tree which can attain a height of $15-25 \mathrm{~m}$. It comes from the Sterculiaceae family and has pneumatophore roots, elliptical leaves, and blooms bell-shaped flowers pink to orange in color [51]. In Bhitarkanika and Sunderbans, India, the leaves, roots, and stems are traditionally used to treat cardiovascular diseases, gastrointestinal disorders (diarrhea, dyspepsia, stomach ache, dysentery, constipation), and skin diseases (rash, eczema, boils, itch, sores) [7,51,89,90]. Ali et al. [91] and Rahmatullah et al. [69] also reported that the whole plant or twig could be used against bloating, diabetes, heart disease, hepatic disorders, goiter, toothache, and oral infection. H. fomes showed antihyperglycemic, antidiabetic, and antinociceptive activities in the methanolic bark extract (Table 7). The ethanolic leaf extract showed excellent antimicrobial activity against E. coli, S. typhi, S. paratyphi, and S. aureus [51]. Rahmatulla et al. [69] conducted the toxicity test on the leaf extract and the \% writhing inhibitions at dosage 250 and $500 \mathrm{mg} / \mathrm{kg}$ was 34.83\% and 59.20\%, respectively. The ethanolic leaf extract, bark extract, and the acetone and aqueous stem extracts were screened for the phytochemical compositions, and the constituents mainly include alkaloids, cardiac glycosides, tannins, steroids, saponins, gums, carbohydrates, proteins, and amino acids, among others (Table 8).

$H$. littoralis comes from the Sterculiaceae family similar to H. fomes and reaches a height of $25 \mathrm{~m}$. It forms pneumatophore root and has dark green leaves with acute apex which are generally $10-23 \mathrm{~cm}$ long and 4-10 cm wide [51]. This species is broadly distributed in Asia mainly in China, Taiwan, Guangdong, Guangxi, Fujian, and Philippines [92]. In Philippines, the sap is traditionally used to counteract fish, arrowhead, and spearhead poisoning and the seed is used to treat diarrhea, dysentery, and hematuria [92]. Wang et al. [126] studied the antioxidant activities on the leaves and roots using three different assays, namely DPPH, HOm and SO. The $\mathrm{IC}_{50}$ value for the leaf extracts for all the three methods are $0.028,0.600$, and $0.606 \mathrm{mg} / \mathrm{mL}$, respectively. Ge et al. [92] also conducted phytochemical screening and isolated four compounds namely 3,5,7-trihydroxychromone-3-O- $\alpha$-L-rhamnopyranoside (245), quercetin-3-O- $\alpha$-L-rhamnopyranoside (246), (2R,3R)-dihydroquercetin-3-O- $\alpha$-L-rhamnopyranoside (247), and kaempferol-3-O- $\alpha$-L-rhamnopyranoside (248) from the ethanolic leaf extract and Revathi et al. [88] reported the presence of alkaloids, tannins, polyphenols, and saponins in the stem, bark, fruit, and leaf extract.

\subsection{Lumnitzera racemosa}

This mangrove tree is used traditionally by the local people of Orissa, India, to treat rheumatism, skin allergies, asthma, diabetes, snake bites, and as a blood purifier [95]. L. racemosa showed antioxidant, cytotoxicity, and anticoagulant properties (Table 7). Cytotoxicity test was conducted on the aqueous leaf extract against Hep G2 cancer cell line using MTT assay. The resulting $\mathrm{IC}_{50}$ value was $26.05 \mu \mathrm{g} / \mathrm{mL}$, and the extract was reported to exhibit potent cytotoxicity activity on the Hep G2 cell lines [127]. The aqueous leaf, methanolic twig, and dichloromethane: methanol stem extracts were screened for phytochemicals and most constituents present were alkaloids, phenols, flavonoids, terpenoids, tannins, sterols, carbohydrates, quinines, saponins, quercetin, and aromatic ester [127,167,177].

\subsection{Kandelia}

K. candel originates from the Rhizophoraceae family and grows to a height of up to $10 \mathrm{~m}$. It has flaky barks with lenticels on its surface and is reddish-brown. The plant produces oval-shaped fruits, $25 \mathrm{~cm}$ long and blooms white flowers [57]. K candel is distributed along the tropical and subtropical coastline of China and from western India to Borneo [93]. The plant is traditionally used to treat 
cardiovascular diseases, cancer, and neurodegenerative disorders. The leaf extract of this mangrove species is reported to possess excellent antioxidant activities. The ethyl acetate hypocotyl extract has an $\mathrm{IC}_{50}$ value of $124.19 \pm 3.02 \mu \mathrm{g} / \mathrm{mL}$ with DPPH assay and an AAE value of $4.39 \pm 3.17 \mathrm{mmol} / \mathrm{g}$ with FRAP (Ferric reducing antioxidant power) assay [93].

K. rheedii has been used for tuberculosis treatment in India [94]. Revathi et al. [88] reported the presence of steroids and triterpenoids in the bark, leaf, and fruit extracts.

\subsection{Nypa fruticans}

$N$. fructicans, also known as Nypa palm, is a 9-m tall prostate-stemmed gregarious palm originating from the Arecaceae family. The leaves have a palm-like structure. It is distributed in Queensland (Australia), India [180], and Malaysia [179]. This mangrove palm is reported to have received little scientific attention. In Malaysia, the local inhabitants used the plant to manage diabetes [96] and in Philippines, the flowers and leaves are traditionally used to treat diabetes and snake bites [61]. The methanolic leaf extract showed antimicrobial activity against E. coli, A. tumefaciens, S. mutans, and S. aureus while the extract was inactive against $A$. flavus and T. rubrum [106]. The antioxidant activity of ethyl acetate extract was investigated for its antioxidant activity using DPPH assay, and the result showed an $\mathrm{IC}_{50}$ value of $2.770 \pm 0.012 \mathrm{mg} / \mathrm{mL}$ [96].

\subsection{Pelliciera rhizophorae}

P. rhizophorae, also known as tea mangrove, is endemic to the coastline of Central America [44]. It comes from the Tetrameristaceae family. It attains a height of up to $20 \mathrm{~m}$, has a buttress root system, and dark-green, elongated, pointed leaves which are $20 \mathrm{~cm}$ long and $5 \mathrm{~cm}$ wide [53]. Bioassay-guided fractionation isolated 10 phytoconstituents, namely $\alpha$-amyrin (46), $\beta$-amyrin (47), ursolic acid (50), oleanolic acid (49), betulinic acid (98), brugierol (157), iso-brugierol (158), kaempferol (79), and quercetin (12). The structures of the isolated compounds were determined by two spectroscopic techniques such as APCI-HR-MS and NMR. Oleanolic acid (49), kaempferol (79), and quercetin (12) showed antiparasitic activity against Leishmania donovani, and their respective $\mathrm{IC}_{50}$ values were $5.3,22.9$, and $3.4 \mu \mathrm{M}$ while $\alpha$-amyrin (46) and betulinic acid (98) exhibited activity against Tripanosoma cruzi and Plasmodium falciparum with the corresponding $\mathrm{IC}_{50}$ values of 19.0 and $18.0 \mu \mathrm{M}$.

\subsection{Rhizophora}

R. apiculata comes from the well-known Rhizophoraceae family. It is $30 \mathrm{~m}$ tall, has stilt roots, and almost smooth bark. The leaves are decussate, have the acute apex and reddish petiole, are $1.5-3 \mathrm{~cm}$ long, and bloom yellow flowers [188]. It is widely distributed across the globe namely Australia, Guam, India, Indonesia, Malaysia, Micronesia, New Caledonia, Papua New Guinea, Philippines, Singapore, Taiwan, Sri Lanka, Maldives, Thailand, Vanuatu, and Vietnam. Folk medicinal practitioners in Tamil Nadu used the whole plant to prevent colitis and inflammatory bowel disease [97] and in Pichavaram region in India, the bark is used to treat amoebiasis, diarrhea, nausea, and vomiting [58]. R. apiculata possesses many pharmacological properties namely antioxidant, antimicrobial and anti-HIV activities. The butanol, ethanolic, ethyl acetate, and water stem extracts were tested for the antioxidant activity using DPPH, ABTS, and HO assays [132]. Lim et al. [133] investigated the antimicrobial activity of the crude bark extract using disc diffusion assay. The extract was found active against 11 microorganisms such as Proteus mirabilis, Acinetobacter calcoaceticus, S. epidermidis, Yersinia enterocolitica, S. aureus, P. aeruginosa, B. cereus, E. coli, B. subtilis, Candida albicans, and Cryptococcus neoformans. However, no fungal activity was reported. Revathi et al. [88] reported the presence of diphatic alcohols, hydrolysable tannins, steroids, triterpenoids, and phenolic compounds in the bark, flower, fruit, and leaf extracts. Gao and Xiao [132] isolated three compounds, namely Lyoniresinol-3 $\alpha-O-\beta$-arabinopyranoside (258), Lyoniresinol-3 $\alpha$-O- $\beta$-rhamnoside (259), and Afzelechin-3-rhamnoside (260) from the twigs, leaves, and barks. HPLC analysis indicated that the three compounds were mainly found in the bark extract with their respective $\%$ as $0.068 \%, 0.066 \%$, and $0.011 \%$. 
R. mucronata, also known as red mangrove, loop-root mangrove, or Asian mangrove, is a $20-25 \mathrm{~m}$ tall mangrove tree and form part of the Rhizoporaceae family. The species has stilt roots buttressing the trunk. It has dark green thick leaves with a distinct mucronate tip and covered with minute black spots on the inferior surface. The mangrove tree produces green fruits with a cigar shape. The flowers are creamy white in color [13]. The plant is found to be present in many countries across the globe. R. mucronata is native to Africa (Egypt, Ethiopia, Kenya, Madagascar, Mauritius, Mozambique, Tanzania, Somalia, South Africa, Sudan), Seychelles island, Asia (India, Papua New Guinea, Sri Lanka, Philippines, Thailand, Taiwan, Vietnam), South Pacific (Solomon Islands, Vanuatu), and Australia (Queensland, Northern Territory). This mangrove species has many beneficial medicinal properties. For instance, in Tamil Nadu, India, the bark or the whole plant is traditionally used to cure angina, dysentery, hematuria, hepatitis, ulcers, diabetes, hemorrhage, vomiting, and nausea. In Mauritius, the local inhabitants use the R. mucronata plant as a traditional medicine against diabetes, hypertension, and also as a natural remedy to reduce the level of urea in the blood. A tea is prepared using root $(5 \mathrm{~cm}$ length) of R. mucronata, 3 whole plants of Bidenspilosa, 10 leaves of $P$. borbonense, bark ( $15 \mathrm{~cm}$ length) of Erythroxylum laurifolium, 15 leaves of Aphloia jobi, and 10 leaves of Antidesma madagascariense. The tea is taken to balance the level of urea in the blood [13]. The root decoction is used to manage diabetes and hypertension while the leaf infusion can be used for fever. The Indonesians traditionally sued the whole plant as a treatment for elephantiasis, haematoma, hepatitis, ulcer, and febrifuge $[60,61]$. In China and Japan, the bark is used against diarrhea [99]. In Papua New Guinea, the local people used the stem to stop constipation, cure fertility and menstruation disorders [59]. R. mucronata possesses many pharmacological properties namely antioxidant, anti-inflammatory, anti-bacterial, antimicrobial, antidiabetic, analgesic, anti-HIV, and anti-cholinesterase properties (Table 7). Chakrarborty and Raola [137] conducted the antioxidant study on the crude chloroform leaf extract using DPPH assay and the resulting $\mathrm{IC}_{50}$ value was $1.38 \pm 0.03 \mathrm{mg} / \mathrm{mL}$, while Suganthy and Devi [100] conducted the same assay to obtain an $\mathrm{IC}_{50}$ value of $47.39 \pm 0.43 \mu \mathrm{g} / \mathrm{mL}$. Interestingly, a study conducted by Hardoko [144] reported that the ripe flour of the fruit contains $7.50 \%$ soluble dietary fiber and $38.60 \%$ insoluble dietary flour. Additionally, the antidiabetic in vivo study conducted showed a decline in the blood glucose level, which, as a result, makes the ripe flour of $R$. mucronata a good functional food for diabetic patients. Recently, Aljaghthmi et al. (2018) showed that the bioactive compounds present in this mangrove species contribute in lowering blood sugar level and boosting insulin production. Pimpliskar et al. (2012) investigated the antimicrobial activity on the ethanolic stem extract. However, to the best of the knowledge of the authors, no other studies were conducted on ripe flour to support or confirm these results obtained by Hardoko [144]. Alikunhi et al. [145] added that the antidiabetic properties of R.mucronata, R.apiculata, and R. annamalayana were due to the presence of the insulin-like protein present in the leaves. $R$. apiculata was more potent compared to the other two Rhizophora species since the results were in equivalence with the control drug, glibenclamide. The extract inhibited activity against E. coli $(16 \mathrm{~mm})$, S.s aureus $(15 \mathrm{~mm})$, S. typhi $(20 \mathrm{~mm})$, S. pyogenes $(12 \mathrm{~mm})$, and P.s aeruginosa $(15 \mathrm{~mm})$, respectively. However, no inhibition was noted against K. pneumonia, P.s vulgaris, and C. albicans. The different plant parts of R. mucronata contain a wide variety of phytochemicals namely condensed tannins, polyphenols, lipids, inositol, gibberellins, alkaloids, tannins, and proteins, among others $[58,60,88,137,167]$ (Table 8 ).

In India, the bark of R. conjugata is used against diabetes [101]. Vadlapuri and Naidu [128] investigated the antimicrobial activity on the crude extract using agar-well diffusion and the extract was active against seven bacterial pathogens namely Acremonium strictum $(7 \mathrm{~mm})$, A. flavus $(8 \mathrm{~mm})$, C. albicans $(11 \mathrm{~mm})$, S.s mutans $(15 \mathrm{~mm})$, S. salivarius $(19 \mathrm{~mm})$, S. aureus $(11 \mathrm{~mm})$, and Lactobacillus acidophilus $(22 \mathrm{~mm})$, respectively. Activity was highest against Lactobacillus acidophilus. Phytochemical screening of the bark and stem extracts showed the presence of anthocyanins, tannins, steroids, and triterpenoids (Table 8).

R. mangle comes from the Rhizophoraceae family and attains a height of $24 \mathrm{~m}$. It has stilt roots, thin and smooth bark grey or grey-brown in color. The leaves are elliptical in shape, thick, shiny green on 
the upper surface, and yellow-green with black spots on the bottom surface [56]. In India, the leaf and bark are used traditionally to manage diabetes $[88,101]$. R. mangle possessed antioxidant and anti-ulcer activities (Table 6). Andrade-Cetto et al. (2017) isolated six compounds from the ethanolic cortex extract, namely Cinchonains Ia and $\mathrm{Ib}$, catechin-3-O-rhamnopyranoside, lyoniside, and nudiposide using NMR, UPLC-DAD-MS, HPLC, and the standard TLC techniques. Revathi et al. [88] and Kandil et al., (2004) reported the presence of tannins, triterpenes, flavonoids, glycosides, quercetin, myricetin, and kaempferol diglycosides in the bark and leaf extract.

R. racemosa originates from the Rhizophoraceae family. It has a height of up to $30 \mathrm{~m}$, stilt roots, and elliptical leaves [26]. Revathi et al. [88] reported the presence of tannins and steroids in the flower and leaf extract of that plant. This mangrove species has been evaluated for its lethal dose $\left(\mathrm{LD}_{50}\right)$ which is commonly used as a toothache remedy by the Nigerian people [102].

$R$. stylosa is a mangrove tree from the Rhizophoraceae family with a height of up to $15 \mathrm{~m}$. Its bark is dark brown to black. It produces ovoid to pear-shaped propagules and is generally $4 \mathrm{~cm}$ long [57]. $\mathrm{Li}$ et al. (2010) isolated eight compounds from the crude stem and twig extract namely (-)-epicatechin (276), 3-O-acetyl (-)-epicatechin (277), 3,3' ,4' ,5,7-O-pentaacetyl (-)-epicatechin (278), (+)-afzelechin (279), (+)-catechin (128), cinchonain Ib (268), and proanthocyanidin B2. Revathi et al. [88] reported the presence of inositols and steroids in the leaves, roots, and seeds extract.

\subsection{Xylocarpus granatum}

This species is a small mangrove plant of height $3-8 \mathrm{~m}$ with a buttress root system. It has a light brown, yellowish, or greenish bark, and is smooth and flaky. The leaves are bright light green to dark green with a round apex [24]. The mangrove species occurs mainly in the Indian Ocean and Southeast Asia [185]. In East Africa and South Asia, the local people use the bark and leaf as a natural remedy for cholera, diarrhea, fever, and malaria [58,104]. X. granatum has many pharmacological activities namely antioxidant, anticancer, antidiarrheal, and antimicrobial (Table 7). Das et al. [104] investigated the antimicrobial activity on the ethanolic stem extract against seven bacterial pathogens, namely E. coli, E. aerogenes, P. aeruginosa, S. typhi, S. aureus, K. pneumonia, and $V$. cholera. The extract was active against all tested microorganisms. Wu et al. [185] isolated three new limonoids, namely 2,3-dideacetylxyloccensin S (281), 30-deacetylxyloccensin W (282), and 7-hydroxy-21b-methoxy-3-oxo-24,25,26,27-tetranortirucalla-1,14-diene-23(21)-lactone (283) from the seed of the Chinese mangrove, X. granatum (Table 8).

\section{Conclusions and Future Perspectives}

This review attempts to project the importance of various mangrove species used traditionally. An overview of their ecological aspects is also given since these plants represent a symbolic plant for the marine ecosystem. The fundamental ecological roles mangroves species play need to be understood to safeguard our environment as these species and their habitats are threatened due to rapid coastal development, extensive aquaculture, climate change scenarios, and overharvesting [9]. So far, there have been piecemeal reviews on mangroves dealing with one aspect or one species at a time but none of them have systematized all the traditionally known mangroves under one review. For instance, Rahmatullah et al. [69] evaluated botanical features and phytochemical profiling of only one mangrove species, B. gymnorhiza. Bandaranayake $[33,65]$ reviewed chemical constituents of mangroves, while Mahmud et al. (2014) targeted only one species, H. fomes, to evaluate its pharmacological properties and ethnomedicinal uses. Ravindran et al. (2005) [58] reviewed the therapeutic importance and phytochemical screening of one genus, Rhizophora, in a book chapter while Shilpi et al., (2012) documented the antinociceptive, anti-inflammatory, and anti-pyretic activities of mangrove plants without detailing on the phytochemical screenings. Kathiresan [189] focused on mangroves from Pichavaram (India) only, while Mondal et al. [50] documented only one species, E. agallocha. Patra and Mohanta [190] have reported only the antimicrobial aspects of a few mangroves, and Simlai and Roy [147] elaborated on biological activities and chemical constituents from mangroves 
in only a specific region of the Sundarban estuary. This review article provides a more extensive coverage on all mangroves by compiling updated information and data on their discovery, ecology and physiological aspects, types, geographical distribution, taxonomy, morphological characteristics, ethnopharmacology, pharmacological activities, and phytochemical evaluation.

Morphologically, mangroves are defined as small trees or shrubs growing along the coastlines in muddy or rocky soils. For instance, Kathiresan and Bingham [6] classified mangroves as halophytes, however Collins, Merriam-Webster, and the Oxford English dictionaries defined mangroves simply as trees or shrubs with tangled roots growing along the coastlines of tropical countries while Spalding [14] generalized mangroves as trees or large shrubs growing in or adjacent to intertidal regions which can easily adapt themselves in their environment. Having said that, it can be concluded that there are still ambiguities in the definition of mangroves and thus require the attention of botanists to properly define the plant. In many countries, particularly in India, people believed that mangroves can cure a wide spectrum of diseases such as rheumatism, diabetes, fever, and gastrointestinal disorders (diarrhea, dysentery, dyspepsia, constipation). Locally, the Mauritian people have used the plant as a traditional medicine for diabetes and hypertension for many years. Interestingly, mangroves are not only important for people but equally significant for animals. For instance, a study conducted by Gardner [16] in Madagascar showed that lemurs use mangroves as their prime natural habitat for sleeping and foraging [16].

From the literature, it is acknowledged that there are 84 mangrove species. However, only 27 species are known to the folklore medicine and not all species have been tested for their pharmacological activities both in vivo and in vitro, which accounts for only about $31 \%$ of mangrove species that have been investigated till date. This rather low percentage can be linked to either a poor interest from the researchers' side on these particular plants or because these plants are considered as endangered species in some countries. Therefore, this might have created a gap between traditional medicines and the interest in developing drugs derived from mangroves. Consequently, to fill the gap between traditional medicines and pharmaceutics, more research is needed to provide a greater range of potential cures against a panel of diseases.

So far, we have seen that mangrove species has a long history in traditional medicine/ ethnopharmalogy and is still widely used because of a wide array of potential sources of natural compounds. Several classes of bioactive substances have been isolated and identified and investigations on different metabolic activities have been performed both in vitro and in vivo. While we present and discussed herein evidence in connection with mangrove species and their beneficial medicinal properties, there are still doubts as to how far these bioactive compounds can be used as direct disease management agents. There is no conclusive report of human trials and up to what extent these beneficial medicinal properties are substantiated warrant further investigation. For proper ethnopharmacological use of mangroves, we believe there should be more direct scientific evidence substantiated with more clinical-based research with rationale impact assessed on human health.

A deeper scientific understanding of the mechanisms of those compounds, their molecular targets, and any drug interaction should be further investigated. Well-designed in vivo tests and randomized controlled clinical studies should be carried out to obtain statistically significant outcomes. There is also a dire need to ensure the efficacy and safety of mangrove preparations and not direct their use solely based on people's perceptions. Other pertinent questions that must be delved in are: How far can these mangroves be further exploited on a commercial scale by pharmaceutical companies? What are the optimized methods of extraction and characterization? What are the risk levels or adverse human effects? What types of pharmacological evaluations must be carried out to confirm activity of mangrove ingredients?

Author Contributions: M.F. conceptualized the study. S.N. conducted the data mining, field work, and prepared the first draft. All authors were involved in improving and polishing the manuscript.

Funding: The author KRRR thanks the DST-SERB, New Delhi for financial support in the form of postdoctoral fellowship (File. No. PDF/2017/001166/LS). The authors KRRR and SKP sincerely acknowledge the computational 
and bioinformatics facility provided by the Alagappa University Bioinformatics Infrastructure Facility (funded by DBT, GOI; File No. BT/BI/25/012/2012, BIF). The authors KRRR and SKP also thank RUSA 2.0 (F. 24-51/2014-U, Policy (TN Multi-Gen), Dept of Edn, GoI).

Conflicts of Interest: The authors declare no conflict of interest.

\section{Abbreviations}

$\begin{array}{ll}\text { APCI/HR/MS } & \text { Atmospheric pressure chemical ionisation high resolution mass spectrometry } \\ \text { ABTS } & \text { 2,2-azino-bis-3-ethyl benzthiazoline-6-sulfonic acid radical } \\ \text { COX-2 } & \text { Cyclooxigenase 2 } \\ \text { DPPH } & \text { 1-diphenyl-2-picryhydrazyl } \\ \text { EBSCO } & \text { Elton B. Stephens Co } \\ \text { FRAP } & \text { Ferric reducing antioxidant power } \\ \text { GC/MS } & \text { Gas chromatography mass spectrometry } \\ \text { HRESI/MS } & \text { High resolution electrospray ionisation mass spectrometry } \\ \text { HO } & \text { Hydroxyl } \\ \text { IC } 50 & \text { Inhibitory concentration 50 } \\ \text { NR } & \text { No result } \\ \text { NI } & \text { Not indicated } \\ \text { PROSEA } & \text { Plant resources south-east Asia } \\ \text { REMA } & \text { Resazurin microtitre assay } \\ \text { SI } & \text { Selective index } \\ \text { SO } & \text { Superoxide } \\ \text { UPLC/DAD/MS } & \text { Ultra high performance liquid chromatography diode array detector tandem } \\ & \text { mass spectrometry }\end{array}$

\section{References}

1. Ernst, M.; Saslis-Lagoudakis, C.H.; Grace, O.M.; Nilsson, N.; Simonsen, H.T.; Horn, J.W.; Rønsted, N. Evolutionary prediction of medicinal properties in the genus Euphorbia L. Sci. Rep. 2016, 6, 30531. [CrossRef] [PubMed]

2. Mahdi, J.G. Medicinal potential of willow: A chemical perspective of aspirin discovery. J. Saudi. Chem. Soc. 2010, 14, 317-322. [CrossRef]

3. Daugan, M.; Wojcicki, A.D.; d'Hayer, B.; Boudy, V. Metformin: An anti-diabetic drug to fight cancer. Pharmacol. Res. 2016, 113, 675-685. [CrossRef] [PubMed]

4. Surapaneni, M.S.; Das, S.K.; Das, N.G. Designing paclitaxel drug delivery systems aimed at improved patient outcomes: Current status and challenges. Int. Sch. Res. Notices 2012, 623139, 1-15. [CrossRef]

5. Willis, K.J. State of the World's Plants 2017; Royal Botanic Gardens, Kew: London, UK, 2017.

6. Kathiresan, K.; Bingham, B.L. Biology of mangroves and mangrove ecosystems. Adv. Mar. Biol. 2001, 40, 81-251.

7. Thatoi, H.; Samantaray, D.; Das, S.K. The genus Avicennia, a pioneer group of dominant mangrove plant species with potential medicinal values: A review. Front. Life Sci. 2016, 9, 267-291. [CrossRef]

8. Hamilton, S.E.; Casey, D. Creation of a high spatio-temporal resolution global database of continuous mangrove forest cover for the 21st century (cgmfc-21). Glob. Ecol. Biogeogr. 2016, 25, 729-738. [CrossRef]

9. Yeo, S. Save Mangroves for People, Planet and the Economy, Says UN. 2014. Available online: https://www. climatechangenews.com/2014/09/30/save-mangroves-for-people-planet-and-the-economy-says-un/ (accessed on 02 August 2018).

10. Van Lavieren, H.; Spalding, M.; Alongi, D.M.; Kainuma, M.; Clüsener-Godt, M.; Adeel, Z. Securing the Future of Mangroves; United Nations University, Institute for Water, Environment and Health: Hamilton, ON, Canada, 2012.

11. Naidoo, G. The mangroves of South Africa: An ecophysiological review. S. Afr. J. Bot. 2016, 107, 101-113. [CrossRef]

12. MacNae, W. A general account of the fauna and flora of mangrove swamps and forests in the indo-west-pacific region. In Advances in Marine Biology; Elsevier: Amsterdam, The Netherlands, 1969; Volume 6, pp. 73-270. 
13. Gurib-Fakim, A.; Brendler, T. Medicinal and Aromatic Plants of Indian Ocean Islands: Madagascar, Comoros, Seychelles and Mascarenes; Medpharm GmbH Scientific Publishers: Stuttgart, Germany, 2004.

14. Spalding, M.; Kainuma, M.; Collins, L. World Atlas of Mangroves. A Collaborative Project of Itto, Isme, Fao, Unep-Wcmc; Earthscan: London, UK, 2010.

15. Schneider, P. The discovery of tropical mangroves in graeco-roman antiquity: Science and wonder. J. Hakluyt Soc. 2011. 1-16. Available online: https://www.academia.edu/7635491/The_Discovery_of_Tropical_ Mangroves_in_Graeco-Roman_Antiquity_Science_and_Wonder (accessed on 16 April 2019).

16. Gardner, C.J. Use of mangroves by lemurs. Int. J. Primatol. 2016, 37, 317-332. [CrossRef]

17. Yong, J. Origin of Mangroves \& Mangrove Diversity 2018. Available online: http://mangroveactionproject. org/origin-of-mangroves-mangrove-diversity/\#more-2692 (accessed on 02 May 2018).

18. COLLINS (2018) Definition of 'Mangrove'. Available online: https://www.collinsdictionary.com/dictionary/ english/mangrove (accessed on 31 July 2018).

19. Merriam-Webster. Definition of Mangrove. Available online: https://www.merriam-webster.com/dictionary/ mangrove (accessed on 31 July 2018).

20. Portuguese Translation of 'Mangrove'. Available online: https://www.collinsdictionary.com/dictionary/ english-portuguese/mangrove (accessed on 21 March 2018).

21. Dale, N. Flowering Plants: The Santa Monica Mountains, Coastal \& Chaparral Regions of Southern California; Capra Pr: Bakersfield, CA, USA, 1986.

22. WELSH, S.L. 2003 Flora of North America. Available online: http://www.efloras.org/florataxon.aspx?flora id=1\&taxon_id=103110 (accessed on 26 July 2018).

23. Jayatissa, L.P.; Dahdouh-Guebas, F.; Koedam, N. A review of the floral composition and distribution of mangroves in Sri Lanka. Bot. J. Linn. Soc. 2002, 138, 29-43. [CrossRef]

24. Guide to the Mangroves of Singapore. Available online: http://mangrove.nus.edu.sg/guidebooks/contents. htm (accessed on 16 April 2019).

25. Saenger, P.; Hegerl, E.; Davie, J.D. Global Status of Mangrove Ecosystems; International Union for Conservation of Nature and Natural Resources: Gland, Switzerland, 1983.

26. Tomlinson, P.B. The Botany of Mangroves, 2nd ed.; Cambridge University Press: Cambridge, UK, 2016.

27. Mu, M.R.; Jiang, Q.L.; Wang, W.Q. Comparison of leaf chloride content and leaf traits between true mangrove plants and semimangrove plants. J. Plant. Ecol. 2007, 497-504.

28. Mukherjee, A.; Acharya, L.; Mattagajasingh, I.; Panda, P.; Mohapatra, T.; Das, P. Molecular characterization of three heritiera species using aflp markers. Biol. Plant. 2003, 47, 445-448. [CrossRef]

29. Wang, L.; Mu, M.; Li, X.; Lin, P.; Wang, W. Differentiation between true mangroves and mangrove associates based on leaf traits and salt contents. J. Plant. Ecol. 2010, 4, 292-301. [CrossRef]

30. A Working List of All Plant Species. Available online: http://www.theplantlist.org/browse/A/Rhizophoraceae/ (accessed on 30 November 2017).

31. Nebula, M.; Harisankar, H.; Chandramohanakumar, N. Metabolites and bioactivities of Rhizophoraceae mangroves. Nat. Prod. Bioprospect. 2013, 3, 207-232. [CrossRef]

32. Ricklefs, R.E.; Schwarzbach, A.E.; Renner, S.S. Rate of lineage origin explains the diversity anomaly in the world's mangrove vegetation. Am. Nat. 2006, 168, 805-810. [PubMed]

33. Bandaranayake, W. Traditional and medicinal uses of mangroves. Mangroves Salt Marshes 1998, 2, $133-148$. [CrossRef]

34. Duke, N.C. Mangrove floristics and biogeography. In Tropical Mangrove Ecosystems; Wiley: Hoboken, NJ, USA, 1992.

35. Selvam, V.E.P.; Karunagaran, V.M.; Ravishankar, T.; Ramasuburamanian, R. Mangrove Plants of Tamil Nadu; M. S. Swaminathan Research Foundation: Chennai, India, 2004.

36. Saranraj, P.; Sujitha, D. Mangrove medicinal plants: A review. Am.-Eurasian J. Toxicol. Sci. 2015, 7, $146-156$.

37. Giri, C.; Ochieng, E.; Tieszen, L.L.; Zhu, Z.; Singh, A.; Loveland, T.; Masek, J.; Duke, N. Status and distribution of mangrove forests of the world using earth observation satellite data. Glob. Ecol. Biogeogr. 2011, 20, 154-159. [CrossRef]

38. Thomas, N.; Lucas, R.; Bunting, P.; Hardy, A.; Rosenqvist, A.; Simard, M. Distribution and drivers of global mangrove forest change, 1996-2010. PLoS ONE 2017, 12, e0179302. [CrossRef]

39. Appadoo, C. Status of mangroves in Mauritius. J. Coast. Dev. 2003, 7, 1-4. 
40. Sauer, J.D. Effects of recent tropical cyclones on the coastal vegetation of Mauritius. J. Ecol. 1962, 50, $275-290$. [CrossRef]

41. 4th National Report to the Convention on Biological Diversity. Available online: https://www.cbd.int/doc/ world/sg/sg-nr-04-en.pdf (accessed on 25 March 2018).

42. Saranya, A.; Ramanathan, T.; Kesavanarayanan, K.S.; Adam, A. Traditional medicinal uses, chemical constituents and biological activities of a mangrove plant, Acanthus ilicifolius linn. A brief review. Am. Eurasian J. Agric. Environ. Sci. 2015, 15, 243-250.

43. Flora and Fauna Web. Available online: https://florafaunaweb.nparks.gov.sg/ (accessed on 20 November 2017).

44. López, D.; Cherigo, L.; Spadafora, C.; Loza-Mejía, M.A.; Martínez-Luis, S. Phytochemical composition, antiparasitic and $\alpha$-glucosidase inhibition activities from Pelliciera rhizophorae. Chem. Cent. J. 2015, 9, 53. [CrossRef]

45. Hasan, I.; Hussain, M.S.; Millat, M.S.; Sen, N.; Rahman, M.A.; Rahman, M.A.; Islam, S.; Moghal, M.M.R. Ascertainment of pharmacological activities of Allamanda neriifolia hook and Aegialitis rotundifolia roxb used in bangladesh: An in vitro study. J. Tradit. Complement. Med. 2018, 8, 107-112. [CrossRef]

46. Rippey, E.; Rowland, B. Coastal Plants: Perth and the South-West Region; ISBS: Pimpri-Chinchwad, India, 2004.

47. Sahoo, G.; Ansari, Z.; Shaikh, J.B.; Varik, S.U.; Gauns, M. Epibiotic communities (microalgae and meiofauna) on the pneumatophores of Avicennia officinalis (1.). Estuar. Coast. Shelf. 2018, 207, 391-401. [CrossRef]

48. Putih, B. Bruguiera cylindrica. 2013. Available online: http://www.wildsingapore.com/wildfacts/plants/ mangrove/bruguiera/cylindrica.htm (accessed on 16 December 2017).

49. Soepadmo, E.; Saw, L.; Chung, R. Tree Flora of Sabah and Sarawak: Volume 5; Forest Research Institute Malaysia (FRIM): Selangor, Malaysia, 2004.

50. Mondal, S.; Ghosh, D.; Ramakrishna, K. A complete profile on blind-your-eye mangrove Excoecaria agallocha 1.(euphorbiaceae): Ethnobotany, phytochemistry, and pharmacological aspects. Pharmacogn. Rev. 2016, 10, 123. [CrossRef] [PubMed]

51. Mahmud, I.; Islam, M.K.; Saha, S.; Barman, A.K.; Rahman, M.M.; Anisuzzman, M.; Rahman, T.; Al-Nahain, A.; Jahan, R.; Rahmatullah, M. Pharmacological and ethnomedicinal overview of Heritiera fomes: Future prospects. Int. Sch. Res. Notices 2014, 2014, 938543. [CrossRef] [PubMed]

52. China, F.O. Nypa fruticans. 1779. Available online: http://www.efloras.org/florataxon.aspx?flora_id=2\& taxon_id=220009317 (accessed on 12 December 2017).

53. Mangroves. Available online: http://www.mangrove.at/pelliciera-rhizophorae_tea-mangrove.html (accessed on 15 January 2018).

54. Yi, X.-X.; Deng, J.-G.; Gao, C.-H.; Hou, X.-T.; Li, F.; Wang, Z.-P.; Hao, E.-W.; Xie, Y.; Du, Z.-C.; Huang, H.-X. Four new cyclohexylideneacetonitrile derivatives from the hypocotyl of mangrove (Bruguiera gymnorrhiza). Molecules 2015, 20, 14565-14575. [CrossRef]

55. Rhizophora apiculata (PROSEA). Available online: https://uses.plantnet-project.org/en/Rhizophora_apiculata_ (PROSEA) (accessed on 20 December 2018).

56. Duke, J. Rhizophora mangle 1. 1983. Available online: https://www.hort.purdue.edu/newcrop/duke_energy/ Rhizophora_mangle.html (accessed on 18 December 2017).

57. Soepadmo, E.W. Tree Flora of Sabah and Sarawak, 1st ed.; Forest Research Institute Malaysia: Kuala Lumpur, Malaysia, 1995.

58. Ravindran, K.; Venkatesan, K.; Balakrishnan, V.; Chellappan, K.; Balasubramanian, T. Ethnomedicinal studies of pichavaram mangroves of east coast, Tamil nadu. Indian J. Tradit. Knowl. 2005, 4, 409-411.

59. Liebezeit, G.; Rau, M.T. New Guinean mangroves-Traditional usage and chemistry of natural products. Senck. Marit. 2006, 36, 1-10. [CrossRef]

60. Nurdiani, R.; Firdaus, M.; Prihanto, A.A. Phytochemical screening and antibacterial activity of methanol extract of mangrove plant (Rhizophora mucronata) from porong river estuary. J. Basic Sci. Technol. 2012, 1, $27-29$.

61. Rollet, B. Bibliography on Mangrove Research 1600-1975; UNESCO: Paris, France, 1981.

62. Seepana, R.; Perumal, K.; Kada, N.M.; Chatragadda, R.; Raju, M.; Annamalai, V. Evaluation of antimicrobial properties from the mangrove Rhizophora apiculata and bruguiera gymnorrhiza of burmanallah coast, south andaman, india. J. Coast. Life Med. 2016, 4, 475-478. [CrossRef] 
63. Krishnamoorthy, M.; Sasikumar, J.; Shamna, R.; Pandiarajan, C.; Sofia, P.; Nagarajan, B. Antioxidant activities of bark extract from mangroves, Bruguiera cylindrica (1.) blume and Ceriops decandra perr. Indian J. Pharmacol. 2011, 43, 557. [PubMed]

64. Sur, T.K.; Hazra, A.; Hazra, A.K.; Bhattacharyya, D. Antioxidant and hepatoprotective properties of Indian sunderban mangrove Bruguiera gymnorrhiza 1. Leave. J. Basic Clin. Pharm. 2016, 7, 75. [CrossRef] [PubMed]

65. Bandaranayake, W.M. Bioactivities, bioactive compounds and chemical constituents of mangrove plants. Wetl. Ecol. Manag. 2002, 10, 421-452. [CrossRef]

66. Kumar, K.M.S.; Gorain, B.; Roy, D.K.; Samanta, S.K.; Pal, M.; Biswas, P.; Roy, A.; Adhikari, D.; Karmakar, S.; Sen, T. Anti-inflammatory activity of Acanthus ilicifolius. J. Ethnopharmacol. 2008, 120, 7-12. [CrossRef]

67. Babu, B.; Shylesh, B.; Padikkala, J. Antioxidant and hepatoprotective effect of Acanthus ilicifolius. Fitoterapia 2001, 72, 272-277. [CrossRef]

68. Islam, M.A.; Saifuzzaman, M.; Ahmed, F.; Rahman, M.M.; Sultana, N.A.; Naher, K. Antinociceptive activity of methanolic extract of Acanthus ilicifolius linn. Leaves. Turk. J. Pharm. Sci. 2012, 9, 51-60.

69. Rahmatullah, M.; Sadeak, I.; Bachar, S.C.; Hossain, T.; Jahan, N.; Chowdhury, M.H.; Jahan, R.; Nasrin, D.; Rahman, M.; Rahman, S. Brine shrimp toxicity study of different bangladeshi medicinal plants. Adv. Nat. Appl. Sci. 2010, 4, 163-174.

70. Bose, S.; Bose, A. Antimicrobial activity of Acanthus ilicifolius (1.). Indian J. Pharm. Sci. 2008, 70, 821. [CrossRef] [PubMed]

71. Miles, D.H.; Kokpol, U.; Chittawong, V.; Tip-Pyang, S.; Tunsuwan, K.; Nguyen, C. Mangrove forests-the importance of conservation as a bioresource for ecosystem diversity and utilization as a source of chemical constituents with potential medicinal and agricultural value. IUPAC 1998, 70, 1-9.

72. Ray, T. Customary use of mangrove tree as a folk medicine among the sundarban resource collectors. Int. J. Res. Hum. Arts Lit. 2014, 2, 43-48.

73. Raju, G.S.; RahmanMoghal, M.M.; Hossain, M.S.; Hassan, M.M.; Billah, M.M.; Ahamed, S.K.; Rana, S.M. Assessment of pharmacological activities of two medicinal plant of bangladesh: Launaea sarmentosa and Aegialitis rotundifolia roxb in the management of pain, pyrexia and inflammation. Biol. Res. 2014, 47, 55. [CrossRef] [PubMed]

74. Roome, T.; Dar, A.; Naqvi, S.; Choudhary, M.I. Evaluation of antinociceptive effect of Aegiceras corniculatum stems extracts and its possible mechanism of action in rodents. J. Ethnopharmacol. 2011, 135, 351-358. [CrossRef] [PubMed]

75. Roome, T.; Dar, A.; Naqvi, S.; Ali, S.; Choudhary, M.I. Aegiceras corniculatum extract suppresses initial and late phases of inflammation in rat paw and attenuates the production of eicosanoids in rat neutrophils and human platelets. J. Ethnopharmacol. 2008, 120, 248-254. [CrossRef]

76. Thomas, T. In vitro evaluation of antibacterial activity of Acrostichum aureum linn. Indian J. Nat. Prod. Resour. 2012, 31, 135-138.

77. Rajeshwari, E.; Gajendiran; Elamathy, S. Study of preliminary phytochemical analysis and antibacterial activity of selected medicinal plants (Avicennia germinans). Int. J. 2013, 1, 952-954.

78. Khajehzadeh, S.; Behbahani, M. Activity of Avicennia marina methanol extracts on proliferation of lymphocytes and their mutagenicity using ames test and in silico method. J. Mazandaran Univ. Med. Sci. 2016, 26, 32-42.

79. Sumithra, M.; Anbu, J.; Nithya, S.; Ravichandiran, V. Anticancer activity of methanolic leaves extract of Avicennia officinalis on ehrlich ascitis carcinoma cell lines in rodents. Int. J. Pharm. Tech. Res. 2011, 3, 1290-1292.

80. Thirunavukkarasu, P.; Ramanathan, T.; Ramkumar, L.; Shanmugapriya, R.; Renugadevi, G. The antioxidant and free radical scavenging effect of Avicennia officinalis. J. Med. Plants Res. 2011, 5, 4754-4758.

81. Ahmed, F.; Shahid, I.; Gain, N.; Reza, M.; Sadhu, S. Antinociceptive and antidiarrhoeal activities of Bruguiera gymnorrhiza. Orient. Pharm. Exp. Med. 2007, 7, 280-285. [CrossRef]

82. Karimulla, S.; Kumar, B. Antidiabetic and antihyperlipidemic activity of bark of Bruguiera gymnorrhiza on streptozotocin induced diabetic rats. AJPS 2011, 1, 4-7.

83. Haq, M.; Sani, W.; Hossain, A.; Taha, R.M.; Monneruzzaman, K. Total phenolic contents, antioxidant and antimicrobial activities of Bruguiera gymnorrhiza. J. Med. Plants Res. 2011, 5, 4112-4118.

84. Bamroongrugsa, N. Bioactive substances from the mangrove resource. Songklanakarin J. Sci. Technol. 1999, 21, 377-386. 
85. Siemonsma, J.; Piluek, K. Plant resources of south-east Asia. No. 8: Vegetables. 1993. Available online: http://agris.fao.org/agris-search/search.do?recordID=XF2015019284 (accessed on 25 January 2018).

86. Ravi, A.V.; Kathiresan, K. Seasonal-variation in gallotannin from mangroves. IJMS 1990, 19, $224-225$.

87. Premanathan, M.; Nokashima, H.; Kathiresan, K.; Rajendran, N.; Yamamoto, N. In vitro anti human immunodeficiency virus activity of mangrove plants. Indian J. Med. Res. 1996, 103, 278-281.

88. Revathi, P.; Senthinath, T.J.; Thirumalaikolundusubramanian, P.; Prabhu, N. An overview of antidiabetic profile of mangrove plants. Int. J. Pharm. Pharm. Sci. 2014, 6, 1-5.

89. Hossain, M.A.; Panthi, S.; Asadujjaman, M.; Khan, S.A.; Ferdous, F.; Sadhu, S.K. Phytochemical and pharmacological assessment of the ethanol leaves extract of Heritiera fomes buch. Ham.(family-sterculiaceae). J. Porphyr. Phthalocyanines. 2013, 2, 95-101.

90. Patra, J.K.; Thatoi, H. Anticancer activity and chromatography characterization of methanol extract of Heritiera fomes buch. Ham.; a mangrove plant from bhitarkanika, india. OPEM 2013, 13, 133-142. [CrossRef]

91. Ali, M.; Nahar, K.; Sintaha, M.; Khaleque, H.N.; Jahan, F.I.; Biswas, K.R.; Swarna, A.; Monalisa, M.N.; Jahan, R.; Rahmatullah, M. An evaluation of antihyperglycemic and antinociceptive effects of methanol extract of Heritiera fomes buch-ham.(sterculiaceae) barks in swiss albino mice. Adv. Nat. Appl. Sci. 2011, 5, 116-121.

92. Ge, L.; Li, Y.; Yang, K.; Pan, Z. Chemical constituents of the leaves of Heritiera littoralis. Chem. Nat. Compd. 2016, 52, 702-703. [CrossRef]

93. Wei, S.-D.; Zhou, H.-C.; Lin, Y.-M. Antioxidant activities of extract and fractions from the hypocotyls of the mangrove plant Kandelia candel. Int. J. Mol. Sci. 2010, 11, 4080-4093. [CrossRef] [PubMed]

94. Zaman, A. Docking studies and network analyses reveal capacity of compounds from Kandelia rheedii to strengthen cellular immunity by interacting with host proteins during tuberculosis infection. Bioinformation 2012, 8, 1012. [CrossRef] [PubMed]

95. Pattanaik, C.; Reddy, C.; Dhal, N.; Das, R. Utilisation of mangrove forests in bhitarkanika wildlife sanctuary, orissa. Indian J. Tradit. Knowl. 2008, 7, 598-603.

96. Yusoff, N.A.; Yam, M.F.; Beh, H.K.; Razak, K.N.A.; Widyawati, T.; Mahmud, R.; Ahmad, M.; Asmawi, M.Z. Antidiabetic and antioxidant activities of Nypa fruticans wurmb. Vinegar sample from Malaysia. Asian Pac. J. Trop. Biomed. 2015, 8, 595-605. [CrossRef] [PubMed]

97. Prabhu, V.; Guruvayoorappan, C. Protective effect of marine mangrove Rhizophora apiculata on acetic acid induced experimental colitis by regulating anti-oxidant enzymes, inflammatory mediators and nuclear factor-kappa b subunits. Int. J. Immunopharmacol. 2014, 18, 124-134.

98. Premanathan, M.; Arakaki, R.; Izumi, H.; Kathiresan, K.; Nakano, M.; Yamamoto, N.; Nakashima, H. Antiviral properties of a mangrove plant, Rhizophora apiculata blume, against human immunodeficiency virus. Antiviral Res. 1999, 44, 113-122. [CrossRef]

99. Kusuma, S.; Kumar, P.A.; Boopalan, K. Potent antimicrobial activity of Rhizophora mucronata. J. Ecobiotechnol. 2011, 3, 40-41.

100. Suganthy, N.; Pandima Devi, K. In vitro antioxidant and anti-cholinesterase activities of Rhizophora mucronata. Pharm. Biol. 2016, 54, 118-129. [CrossRef]

101. Arora, K.; Nagpal, M.; Jain, U.; Jat, R.; Jain, S. Mangroves: A novel gregarious phyto medicine for diabetes. Int. J. Res. Dev. Pharm. Lif. Sci. 2014, 3, 1244-1257.

102. Angalabiri-Owei, B.; Isirima, J. Evaluation of the lethal dose of the methanol extract of Rhizophora racemosa leaf using karbers method. Afr. J. Cell Pathol. 2014, 2, 65-68. [CrossRef]

103. Dossou-Yovo, H.; Fifanou, G.V.; Sinsin, B. Ethnobotanical survey of mangrove plant species used as medicine from ouidah to grand-popo districts, southern Benin. Am. J. Ethnomed. 2017, 4, 1-6.

104. Das, S.; Samantaray, D.; Thatoi, H. Ethnomedicinal, antimicrobial and antidiarrhoeal studies on the mangrove plants of the genus Xylocarpus: A mini review. J. Bioanal. Biomed. 2014, 12, 004.

105. Banerjee, D.; Chakrabarti, S.; Hazra, A.K.; Banerjee, S.; Ray, J.; Mukherjee, B. Antioxidant activity and total phenolics of some mangroves in sundarbans. Afr. J. Biotechnol. 2008, 7, 805-810.

106. Bakshi, M.; Chaudhuri, P. Antimicrobial potential of leaf extracts of ten mangrove species from Indian sundarban. Int. J. Pharm. Biol. Sci. 2014, 5, 294-304.

107. Reddy, A.R.K.; Grace, J.R. Anticancer activity of methanolic extracts of selected mangrove plants. Int. J. Pharm. Sci. Res. 2016, 38, 3852-3856. 
108. Janmanchi, H.; Raju, A.; Degani, M.; Ray, M.; Rajan, M. Antituberculosis, antibacterial and antioxidant activities of Aegiceras corniculatum, a mangrove plant and effect of various extraction processes on its phytoconstituents and bioactivity. S. Afr. J. Bot. 2017, 113, 421-427. [CrossRef]

109. Rout, P.; Basak, U.C. Antioxidant properties in leaf and root extracts of some medicinally important mangrove species of Odisha coast. Am. J. Pharm. Tech. Res. 2012, 4, 1-13.

110. Shafie, M.M.; Forghani, A.H.; Moshtaghiyan, J. Anti-inflammatory effects of hydro-alcoholic extracts of mangrove (Avicennia marina) and vitamin c on arthritis rats. Bull. Environ. Pharm Life Sci. 2013, 2, 32-37.

111. Karami, L.; Majd, A.; Mehrabian, S.; Nabiuni, M.; Salehi, M.; Irian, S. Antimutagenic and anticancer effects of Avicennia marina leaf extract on Salmonella typhimurium ta100 bacterium and human promyelocytic leukaemia hl-60 cells. Sci. Asia 2012, 38, 349-355. [CrossRef]

112. Sharief, M.N.; Umamaheswararao, V. Antibacterial activity of stem and root extracts of Avicennia officinalis 1. Int. J. Appl. Pharm. 2011, 2, 231-236.

113. Al Maqtari Maher, A. Screening of salt-stress, antioxidant enzyme, and antimicrobial activity of leave extracts of mangroves Avicennia marina 1. From hodaidah, Yemen. J. Stress Physiol. Biochem. 2014, 10, 190-199.

114. Ramanathan, T. Phytochemical characterization and antimicrobial efficiency of mangrove plants Avicennia marina and Avicennia officinalis. Int. J. Pharm. Biol. Arch. 2012, 3, 348-351.

115. Sura, S.; Anbu, J.; Sultan, M.; Uma, B. Antiulcer effect of ethanolic leaf extract of Avicennia officinalis. Pharmacologyonline 2011, 3, 12-19.

116. Hossain, M.H.; Howlader, M.S.I.; Dey, S.K.; Hira, A.; Ahmed, A. Evaluation of diuretic and neuropharmacological properties of the methanolic extract of Avicennia officinalis 1. Leaves from bangladesh. Int. J. Pharm. Phytopharmacol. Res. 2012, 2, 2-6.

117. A Rege, A.; Y Ambaye, R.; A Deshmukh, R. In-vitro testing of anti-hiv activity of some medicinal plants. Int. J. Prod. Res. 2010, 1, 193-199.

118. Hossain, M.L. Medicinal activity of Avicennia officinalis: Evaluation of phytochemical and pharmacological properties. Saudi J. Med. Pharm. Sci. 2016, 2, 250-255.

119. Lakshmi, V.; Sonkar, R.; Khanna, A.K. Antihyperlipidemic and antioxidant activities of Bruguiera cylindrinca (1). Chron Young Sci. 2012, 3, 236. [CrossRef]

120. Gawali, P.; Jadhav, B. Antioxidant activity and antioxidant phytochemical analysis of mangrove species Sonneratia alba and Bruguiera cylindrica. Asian J. Microbiol. Biotechnol. Environ. Sci. 2011, 13, 257-261.

121. Barik, R.; Sarkar, R.; Biswas, P.; Bera, R.; Sharma, S.; Nath, S.; Karmakar, S.; Sen, T. 5,7-dihydroxy-2-(3-hydroxy-4,5-dimethoxy-phenyl)-chromen-4-one-a flavone from Bruguiera gymnorrhiza displaying anti-inflammatory properties. Indian J. Pharmacol. 2016, 48, 304. [PubMed]

122. Uddin, S.J.; Grice, I.D.; Tiralongo, E. Cytotoxic effects of Bangladeshi medicinal plant extracts. Evid. Based Complement. Altern. Med. 2011, 2011, 1-7. [CrossRef]

123. Bunyapraphatsara, N.; Jutiviboonsuk, A.; Sornlek, P.; Therathanathorn, W.; Aksornkaew, S.; Fong, H.H.; Pezzuto, J.M.; Kosmeder, J. Pharmacological studies of plants in the mangrove forest. Thai J. Phytopharm. 2003, 10, 1-12, 2546.

124. Abeysinghe, P.D. Antibacterial activity of some medicinal mangroves against antibiotic resistant pathogenic bacteria. Indian J. Pharm. Sci. 2010, 72, 167-172. [CrossRef]

125. Hossain, H.; Moniruzzaman, S.; Nimmi, I.; Kawsar, H.; Hossain, A.; Islam, A.; Jahan, I.A. Anti-inflammatory and antioxidant activities of the ethanolic extract of Ceriops decandra (griff.) ding hou bark. Orient. Pharm. Exp. Med. 2011, 11, 215-220. [CrossRef]

126. Wang, Y.; Zhu, H.; Tam, N.F.Y. Polyphenols, tannins and antioxidant activities of eight true mangrove plant species in south China. Plant Soil 2014, 374, 549-563. [CrossRef]

127. Paul, T.; Ramasubbu, S. The antioxidant, anticancer and anticoagulant activities of Acanthus ilicifolius 1 . Roots and Lumnitzera racemosa willd. Leaves, from southeast coast of India. J. Appl. Pharm. Sci. 2017, 7, 081-087.

128. Vadlapudi, V.; Naidu, K.C. In vitro bioefficiency of marine mangrove plant activity of Rhizophora conjugata. Int. J. Pharma. Tech. Res. 2009, 1, 1598-1600.

129. SÁnchez Perera, L.M.; Batista, N.Y.; Rodríguez, A.; Farrada, F.; Bulnes, C. Gastric and duodenal antiulcer effects of Rhizophora mangle. Pharm. Biol. 2004, 42, 225-229. [CrossRef]

130. Sánchez, J.C.; García, R.F.; Cors, M.T.M. 1, 1-diphenyl-2-picrylhydrazyl radical and superoxide anion scavenging activity of Rhizophora mangle (1.) bark. Pharmacog. Res. 2010, 2, 279. [CrossRef] 
131. Zhang, L.-L.; Lin, Y.-M.; Zhou, H.-C.; Wei, S.-D.; Chen, J.-H. Condensed tannins from mangrove species Kandelia candel and Rhizophora mangle and their antioxidant activity. Molecules 2010, 15, 420-431. [CrossRef]

132. Gao, M.; Xiao, H. Activity-guided isolation of antioxidant compounds from Rhizophora apiculata. Molecules 2012, 17, 10675-10682. [CrossRef]

133. Lim, S.; Darah, I.; Jain, K. Antimicrobial activities of tannins extracted from Rhizophora apiculata barks. J. Trop. For. Sci. 2006, 18, 59-65.

134. Loo, A.; Jain, K.; Darah, I. Antioxidant activity of compounds isolated from the pyroligneous acid, Rhizophora apiculata. Food Chem. 2008, 107, 1151-1160. [CrossRef]

135. Rahim, A.A.; Rocca, E.; Steinmetz, J.; Kassim, M.J.; Ibrahim, M.S.; Osman, H. Antioxidant activities of mangrove Rhizophora apiculata bark extracts. Food Chem. 2008, 107, 200-207. [CrossRef]

136. Sulaiman, S.; Ibrahim, D.; Kassim, J.; Sheh-Hong, L. Antimicrobial and antioxidant activities of condensed tannin from Rhizophora apiculata barks. J. Chem. Pharm. Res. 2011, 3, 436-444.

137. Chakraborty, K.; Raola, V.K. Two rare antioxidant and anti-inflammatory oleanenes from loop root Asiatic mangrove Rhizophora mucronata. Phytochemistry 2017, 135, 160-168. [CrossRef]

138. Joel, E.L.; Bhimba, V. Isolation and characterization of secondary metabolites from the mangrove plant Rhizophora mucronata. Asian Pac. J. Trop. Biomed. 2010, 3, 602-604. [CrossRef]

139. Gurudeeban, S.; Ramanathan, T.; Satyavani, K. Antimicrobial and radical scavenging effects of alkaloid extracts from Rhizophora mucronata. Pharm. Chem. J. 2015, 49, 34-37. [CrossRef]

140. Ramanathan, T.A.H. In Studies on Analgesic Activity of a Mangrove Species—Rhizophora mucronata Poir. In Proceedings of the Annual International Conference on Advances in Biotechnology (BIOTECH 2011), Bozen, Italy, 12-13 January 2011.

141. Jadhav, R.; Jadhav, B. Evaluation of antimicrobial principles of rhizophora species along mumbai coast. J. Adv. Sci. Res. 2012, 3, 30-33.

142. Wahyuni, W.T.; Darusman, L.K.; Surya, N.K. Potency of rhizopora spp. Extracts as antioxidant and inhibitor of acetylcholinesterase. Procedia Chem. 2015, 16, 681-686. [CrossRef]

143. Manilal, A.; Merdekios, B.; Idhayadhulla, A.; Muthukumar, C.; Melkie, M. An in vitro antagonistic efficacy validation of Rhizophora mucronata. Asian Pac. J. Trop. Dis. 2015, 5, 28-32. [CrossRef]

144. Hardoko, E.S.; Puspitasari, Y.; Amalia, R. Study of ripe Rhizophora mucronata fruit flour as functional food for antidiabetic. Int. Food Res. J. 2015, 22, 953-959.

145. Alikunhi, N.M.; Kandasamy, K.; Manoharan, C.; Subramanian, M. Insulin-like antigen of mangrove leaves and its anti-diabetic activity in alloxan-induced diabetic rats. Nat. Prod. Res. 2012, 26, 1161-1166. [CrossRef]

146. Pandey, A.K.; Gupta, P.P.; Lal, V.K. Hypoglycemic effect of Rhizophora mucronata in streptozotocin induced diabetic rats. J. Complement. Integr. Med. 2014, 11, 179-183. [CrossRef]

147. Simlai, A.; Roy, A. Biological activities and chemical constituents of some mangrove species from sundarban estuary: An overview. Pharmacog. Rev. 2013, 7, 170.

148. Eldeen, I.M.; Effendy, M.A. Antimicrobial agents from mangrove plants and their endophytes. In Méndez-Vilas 9ed. Microbial Pathogens and Strategies for Combating Them: Science, Technology and Education; Formatex Research Center: Badajoz, Spain, 2013; pp. 872-882.

149. Kathiresan, K.; Boopathy, N.S.; Kavitha, S. Coastal vegetation-An underexplored source of anticancer drugs. Indian J. Nat. Prod. Resour. 2006, 5, 115-119.

150. Sithranga Boopathy, N.; Kathiresan, K. Anticancer drugs from marine flora: An overview. J. Oncol. 2010, 214186, 1-18. [CrossRef] [PubMed]

151. Sudirman, S.; Jacoeb, A. Proximate compositions, bioactive compounds and antioxidant activity from large-leafed mangrove (Bruguiera gymnorrhiza) fruit. Int. Food Res. J. 2014, 21, 2387-2391.

152. Rahman, M.; Ahmed, A.; Shahid, I. Phytochemical and pharmacological properties of Bruguiera gymnorrhiza roots extract. Int. J. Pharm. Res. 2011, 3, 63-67.

153. Sreedhar, S.; Christy, P.H. Phytochemical analysis of Rhizophora mucronata. Scrut. Int. Res. Agric. Plant Biotechnol. Biol. Prod. 2015, 2, 39-43.

154. Rohini, R.; Das, A. Antidiarrheal and anti inflammatory activities of lupeol, quercetin, $\beta$-sitosterol, adene-5-en-3-ol and caffeic acid isolated from Rhizophora mucronata bark. Der Pharm. Lett. 2010, 2, 95-101.

155. Fabiyi, O.A. Toxicity of Acanthus ilicifolius (L) fractions against Pratylechus spp. on maize (Zea mays). Alban. J. Agric. Sci. 2015, 14, 149-156. 
156. Ganesh, S.; Jannet Vennila, J. Phytochemical analysis of acanthus ilicifolius and Avicennia officinalis by gc-ms. Res. J. Phytochem. 2011, 5, 60-65. [CrossRef]

157. Lin, N.; Yi, B.; Li, J.; Zhang, W.; Zhang, X. A new sugar ester from the roots of Acanthus ilicifolius. Rec. Nat. Prod. 2017, 11, 74-76.

158. Singh, D.; Aeri, V. Phytochemical and pharmacological potential of Acanthus ilicifolius. J. Pharm. Bioallied Sci. 2013, 5, 17. [PubMed]

159. Tan, D.; Jiang, C.; Tao, Y. Chemical constituents of Acanthus ilicifolius. Chem. Nat. Comp. 2016, 52, 951-952. [CrossRef]

160. Ghosh, D.; Mondal, S.; Ramakrishna, K. Pharmacobotanical, physicochemical and phytochemical characterisation of a rare salt-secreting mangrove Aegialitis rotundifolia roxb.,(plumbaginaceae) leaves: A comprehensive pharmacognostical study. S. Afr. J. Bot. 2017, 113, 212-229. [CrossRef]

161. Fauvel, M.-T.; Bousquet-Melou, A.; Moulis, C.; Gleye, J.; Jensen, S.R. Iridoid glucosides from Avicennia germinans. Phytochemistry 1995, 38, 893-894. [CrossRef]

162. Fauvel, M.-T.; Moulis, C.; Bon, M.; Fourasté, I. A new iridoid glucoside from African Avicennia germinans. Nat. Prod. Lett. 1997, 10, 139-142. [CrossRef]

163. Laphookhieo, S.; Karalai, C.; Ponglimanont, C.; Chantrapromma, K. Pentacyclic triterpenoid esters from the fruits of Bruguiera cylindrica. J. Nat. Prod. 2004, 67, 886-888. [CrossRef] [PubMed]

164. Rahman, S.M.; Kabir, M.Z.; Paul, P.K.; Islam, M.R.; Rahman, S.; Jahan, R.; Rahmatullah, M. A review on a mangrove species from the sunderbans, bangladesh: Bruguiera gymnorrhiza (1.) lam. (Rhizophoraceae). Am.-Eurasian J. Sustain. Agric. 2013, 7, 340-355.

165. Sun, Y.-Q.; Guo, Y.-W. Gymnorrhizol, an unusual macrocyclic polydisulfide from the Chinese mangrove Bruguiera gymnorrhiza. Tetrahedron Lett. 2004, 45, 5533-5535. [CrossRef]

166. Thirunavukkarasu, P.; Asha, S.; Reddy, R.; Priya, D.; Hari, R.; Sudhakar, N. Phytochemical analysis of medicinal mangrove plant species Ceriops decandra. Glob. J. Pharmacol. 2018, 12, 24-30.

167. Anjaneyulu, A.S.; Rao, V.L. Ceriopsins $\mathrm{f}$ and g, diterpenoids from Ceriops decandra. Phytochemistry 2003, 62, 1207-1211. [CrossRef]

168. Poompozhil, S.; Kumarasamy, D. Studies on phytochemical constituents of some selected mangroves. J. Acad. Ind. Res. 2014, 2, 590-592.

169. Nagababu, P.; Umamaheswara, R. Phytochemical, antibacterial and antioxidant evaluation of Ceriops decandra (griff). Ding hou leaf extract. J. Chem. Pharm. Res. 2014, 6, 428.

170. Chen, J.-D.; Yi, R.-Z.; Lin, Y.-M.; Feng, D.-Q.; Zhou, H.-C.; Wang, Z.-C. Characterization of terpenoids from the root of Ceriops tagal with antifouling activity. Int. J. Mol. Sci. 2011, 12, 6517-6528. [CrossRef] [PubMed]

171. Chen, Y.; Wang, W.-J.; Wu, J. Two new dolabranes from the Chinese mangrove Ceriops tagal. J. Asian Nat. Prod. Res. 2016, 18, 41-45. [CrossRef] [PubMed]

172. Du, S.S.; Wang, C.F.; Li, J.; Zhang, H.M.; Liu, Q.Z.; Liu, Z.L.; Deng, Z.W. Antifeedant diterpenoids against tribolium castaneum from the stems and twigs of Ceriops tagal (Rhizophoraceae). Molecules 2011, 16, 6060-6067. [CrossRef] [PubMed]

173. Hu, W.-M.; Li, M.-Y.; Li, J.; Xiao, Q.; Feng, G.; Wu, J. Dolabranes from the Chinese mangrove, Ceriops tagal. J. Nat. Prod. 2010, 73, 1701-1705. [CrossRef] [PubMed]

174. Li, Y.; Liu, J.; Yu, S.; Proksch, P.; Gu, J.; Lin, W. Tnf- $\alpha$ inhibitory diterpenoids from the Chinese mangrove plant Excoecaria agallocha 1. Phytochemistry 2010, 71, 2124-2131. [CrossRef] [PubMed]

175. Ponnapalli, M.G.; Ankireddy, M.; Annam, S.C.V.R.; Ravirala, S.; Sukki, S.; Tuniki, V.R. Unusual ent-isopimarane-type diterpenoids from the wood of Excoecaria agallocha. Tetrahedron Lett. 2013, 54, 2942-2945. [CrossRef]

176. Konishi, T.; Konoshima, T.; Fujiwara, Y.; Kiyosawa, S. Excoecarins d, e, and k, from Excoecaria agallocha. J. Nat. Prod. 2000, 63, 344-346. [CrossRef] [PubMed]

177. DeSouza, L.; Wahidullah, S. Antibacterial phenolics from the mangrove Lumnitzera racemosa. Indian J. Mar. Sci. 2010, 39, 294-298.

178. Jasna, T.; Chandra, P.R.; Khaleel, K. Preliminary phytochemical screening and gc ms analysis of chloroform extract of Kandelia candel (1.) druce. Int. J. Pharm. Sci. Res. 2017, 8, 3530-3533.

179. Prasad, N.; Yang, B.; Kong, K.W.; Khoo, H.E.; Sun, J.; Azlan, A.; Ismail, A.; Romli, Z.B. Phytochemicals and antioxidant capacity from Nypa fruticans Wurmb. Fruit. Evid. Based Complement. Altern. Med. 2013, 2013. [CrossRef] [PubMed] 
180. Ebana, R.; Etok, C.; Edet, U. Phytochemical screening and antimicrobial activity of Nypa fruticans harvested from Oporo River in the niger delta region of nigeria. Int. J. Innov. Appl. Stud. 2015, 10, 1120.

181. Balasubramanian, V.; Rajesh, P.; Rajaram, R.; Kannan, V.R. A review on Rhizophora genus: Therapeutically important perspective phytochemical constituents. In Bioactive Phytochemicals: Perspectives for Modern Medicine; Gupta, V.K., Ed.; Daya Publishing House: New Delhi, India, 2015; Volume 3.

182. Kandil, F.; Grace, M.; Seigler, D.; Cheeseman, J. Polyphenolics in Rhizophora mangle 1. Leaves and their changes during leaf development and senescence. Trees 2004, 18, 518-528. [CrossRef]

183. Andrade-Cetto, A.; Escandón-Rivera, S.M.; Torres-Valle, G.M.; Quijano, L. Phytochemical composition and chronic hypoglycemic effect of Rhizophora mangle cortex on stz-na-induced diabetic rats. Rev. Bras. Farmacogn. 2017, 27, 744-750. [CrossRef]

184. Martins, J.N.; Figueiredo, F.S.; Martins, G.R.; Leitão, G.G.; Costa, F.N. Diterpenes and a new benzaldehyde from the mangrove plant Rhizophora mangle. Rev. Bras. Farmacogn. 2017, 27, 175-178. [CrossRef]

185. Wu, Y.B.; Liu, D.; Liu, P.Y.; Yang, X.M.; Liao, M.; Lu, N.N.; Sauriol, F.; Gu, Y.C.; Shi, Q.W.; Kiyota, H. New limonoids from the seeds of Xylocarpus granatum. Helv. Chim. Acta 2015, 98, 691-698. [CrossRef]

186. Krauss, K.W.; Allen, J.A. Influences of salinity and shade on seedling photosynthesis and growth of two mangrove species, Rhizophora mangle and Bruguiera sexangula, introduced to Hawaii. Aquat. Bot. 2003, 77, 311-324. [CrossRef]

187. Harvey, P. Australian Mangrove and Saltmarsh Resource: Bruguiera sexangula. Available online: https: //coastalresearch.csiro.au/?q=node/119 (accessed on 16 April 2019).

188. Use, P. Rhizophora apiculata (prosea). 2016. Available online: https://uses.plantnet-project.org/en/Rhizophora apiculata_(PROSEA) (accessed on 20 February 2018).

189. Kathiresan, K. A review of studies on pichavaram mangrove, southeast India. Hydrobiologia 2000, 430, 185-205. [CrossRef]

190. Patra, J.K.; Mohanta, Y.K. Antimicrobial compounds from mangrove plants: A pharmaceutical prospective. Chin. J. Integr. Med. 2014, 20, 311-320. [CrossRef] [PubMed]

(C) 2019 by the authors. Licensee MDPI, Basel, Switzerland. This article is an open access article distributed under the terms and conditions of the Creative Commons Attribution (CC BY) license (http://creativecommons.org/licenses/by/4.0/). 